\title{
COLLECTIVE CHARGE- AND SPIN-DENSITY EXCITATIONS IN SEMICONDUCTOR QUANTUM WELLS
}

\author{
A Dissertation presented to \\ the Faculty of the Graduate School \\ at the University of Missouri
}

In Partial Fulfillment

of the Requirements for the Degree

Doctor of Philosophy

by

SHAHRZAD KARIMI

Prof. Carsten Ullrich, Dissertation Supervisor

DECEMBER 2017 
(C) Copyright by Shahrzad Karimi 2017

All Rights Reserved 
The undersigned, appointed by the Dean of the Graduate School, have examined the dissertation entitled:

\section{COLLECTIVE CHARGE- AND SPIN-DENSITY EXCITATIONS IN SEMICONDUCTOR QUANTUM WELLS}

presented by Shahrzad Karimi, a candidate for the degree of Doctor of Philosophy and hereby certify that, in their opinion, it is worthy of acceptance.

Prof. Carsten Ullrich

Prof. Giovanni Vignale

Prof. Sashi Satpathy

Prof. Matthew Maschmann 


\section{ACKNOWLEDGMENTS}

I would like to thank my $\mathrm{PhD}$ advisor, Professor Carsten Ullrich, for supporting me during these past five years. Carsten is one of the smartest people I know and he has one of the most organized minds. He always focuses at one thing at a time, and at the end of the day he is able to accomplish everything on his to-do list in the best way possible. Whether he is in his office or not, he has always been available to answer to all of my questions. Carsten helped me grow in confidence. I remember once I came to his office and was not sure about one of my calculations and I was constantly comparing my calculations with others. He told me to have confidence in

yourself and in what you worked hard for. Being able to work under Carsten is such a privilege, and the knowledge I have gained will never compare to anything else.

My thanks also go to the members of my committee, Prof. Sashi Satpathy, Prof Giovanni Vignale, and Prof. Matthew Maschmann for reading previous drafts of this dissertation and providing many valuable comments.

Last, but not least, I would like to thank my husband. Masoud has faith in me even when I didn't believe in myself. During these past eight years, he has always been there for me. There are no words to convey how much I love him. 


\section{TABLE OF CONTENTS}

ACKNOWLEDGMENTS ................ ii

LIST OF FIGURES $\ldots \ldots \ldots \ldots \ldots \ldots$ vi

ABSTRACT ..................... xiii

\section{CHAPTER}

1 Introduction ..................... 1

2 Three- to two-dimensional crossover in time-dependent densityfunctional theory $\ldots \ldots \ldots \ldots \ldots \ldots \ldots \ldots \ldots \ldots \ldots \ldots$

2.1 Theoretical background . . . . . . . . . . . . . . . . 11

2.1.1 Quantum well model . . . . . . . . . . . . . . . . . . . 11

2.1.2 Excitations within linear-response TDDFT . . . . . . . 13

2.1 .3 Exchange kernels . . . . . . . . . . . . . . . . 16

2.2 Results and Discussions $\ldots \ldots \ldots \ldots$

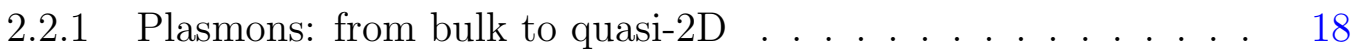

2.2.2 2D Limit of intersubband plasmons . . . . . . . . . . . 21

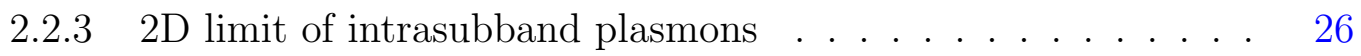

2.3 Conclusions . . . . . . . . . . . . . . . . . . . . . . . . 32

3 Spin precession and spin waves in a chiral electron gas: beyond Larmor's theorem . . . . . . . . . . . . . . . 35

3.1 Larmor's theorem . . . . . . . . . . . . . . . . . . . . . . . . . . 39

3.1.1 Long-wavelength limit of spin waves a 2DEG . . . . . . . . 39 
3.1 .2 TDDFT perspective $\ldots \ldots \ldots \ldots \ldots \ldots \ldots$

3.2 Quantum well with in-plane magnetic field and SOC $\ldots \ldots \ldots$

3.3 Spin-flip waves dispersion $\ldots \ldots \ldots \ldots \ldots$

3.3.1 Linear-response formalism . . . . . . . . . . . . . . . 51

3.3.2 Beyond Larmor's theorem: leading SOC corrections . . . . . 56

3.4 Results and discussion $\ldots \ldots \ldots \ldots \ldots$

3.4.1 Electronic Raman scattering . . . . . . . . . . . . . . . 58

3.4 .2 Comparison with theory . . . . . . . . . . . . . . 62

3.4.3 Density dependence of $E_{0} \ldots \ldots \ldots 6$

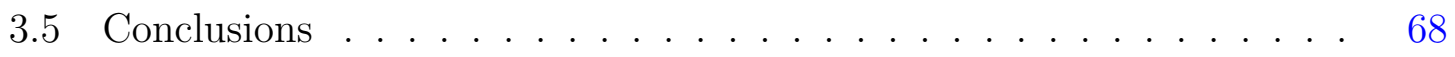

4 Spin-helix Larmor mode . . . . . . . . . . . . . 70

4.1 Results . . . . . . . . . . . . . . . . . . 72

4.1.1 2DEG with spin-orbit coupling in a magnetic field: the spin helix 72

4.1.2 Spin-helix Larmor mode . . . . . . . . . . . . . . 80

4.1 .3 Experimental schemes . . . . . . . . . . . . . . 83

4.2 Discussion . . . . . . . . . . . . . . . . . . . 85

4.3 Methods . . . . . . . . . . . . . . . . . . . . 87

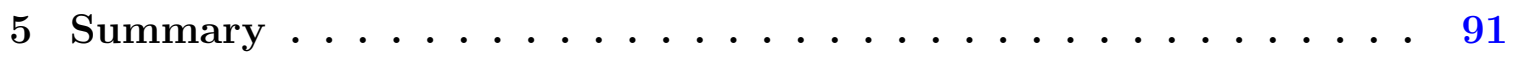
APPENDIX

A The PBE exchange kernel $\ldots \ldots \ldots \ldots \ldots \ldots$

A.1 PBE exchange energy . . . . . . . . . . . . . . . 94

A.2 PBE exchange potential $\ldots \ldots \ldots \ldots \ldots \ldots$ 
A.3 PBE exchange kernel . . . . . . . . . . . . . . . . . . . 96

B The PGG kernel for quasi-2DEGs . . . . . . . . . . . 99

C ISTLS in the exchange-only limit . . . . . . . . . . . 102

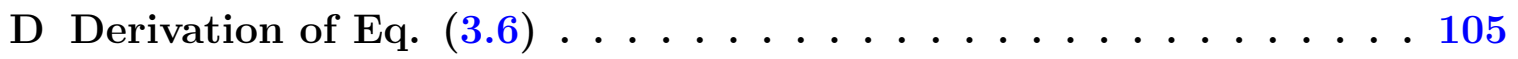

E In-plane anisotropy of the g-factor . . . . . . . . . 115 BIBLIOGRAPHY . . . . . . . . . . . . . 120

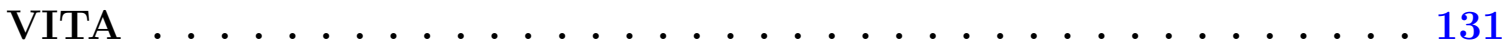




\section{LIST OF FIGURES}

Figure

1.1 Left: Combining two semiconductor materials, A and B, with different band gaps, gives rise to a potential well in conduction and valence band. The electronic levels within the well are quantized into so-called subbands. Here, three subband levels $\varepsilon_{1}, \varepsilon_{2}$, and $\varepsilon_{3}$ are shown. Right: intersubband excitations where the first subband is occupied up to the conduction band Fermi level $\varepsilon_{F} . \ldots \ldots \ldots \ldots$

1.2 Left: Single-particle excitation of a 2DEG which is not spin polarized. The excitation is between states inside the Fermi circle $\varepsilon_{F}$ to states outside. Right: a 2DEG in the presence of a magnetic field, which lifts the spin degeneracy and splits the bands by the effective Zeeman energy $Z^{*}$. The thin blue arrow indicates a single-particle spin-flip

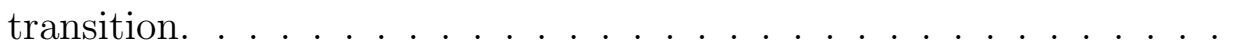

1.3 Symmetry of the Rashba and Dresselhaus spin-orbit effective magnetic fields. Note that if both effects are present, the net spin-orbit field strength has elliptic symmetry. . . . . . . . . . . . . . 
2.1 Illustrations of intersubband (top) and intrasubband (bottom) plasmon excitations with wavevector $q_{\|}$in a quantum well with conduction band Fermi level $\varepsilon_{F}$ in the lowest subband. Intersubband plasmons involve collective transitions between two subbands, leading to density oscillations of the quasi-2D electron system perpendicular to the quantum well plane. Intrasubband plasmons (collective transitions within the lowest subband) are characterized by density oscillations and currents flowing along the plane. . . . . . . . . . . . . . .

2.2 Photoabsorption cross section for $q_{\|}=0$ intersubband excitations in quantum wells. Left panels: charge-density excitations. Right panels: spin-density excitations. Insets: density profiles at given values of $N_{\text {occ }}$. The calculations were done with Eq. (2.14), setting $q_{\|}=0$ and using the 3D ALDA exchange kernel to obtain $n_{1 \sigma} \ldots \ldots \ldots \ldots \ldots$

2.3 Intersubband plasmon dispersions $\Omega\left(q_{\|}\right)$, for $N_{s}=10^{12} \mathrm{~cm}^{-2}$ and well widths $100 \AA$ and $40 \AA$. The black full lines indicate the intersubband p-h continuum. The RPA only gives intersubband charge plasmons; ALDA, PBE and PGG give both charge (full lines) and spin plasmons (dashed lines). ALDA and PBE break down when their charge plasmons falls below the $\mathrm{p}-\mathrm{h}$ continuum. $\ldots \ldots \ldots \ldots \ldots$ 
2.4 Intersubband plasmon energies, at $q_{\|}=0$, versus well width $L$, for $N_{s}=10^{12} \mathrm{~cm}^{-2}$. The horizontal line indicates the lowest p-h transition $\omega_{21}$ (all energies are scaled by $L^{2}$ ). The RPA only gives intersubband charge plasmons; ALDA, PBE and PGG give both charge (full lines) and spin plasmons (dashed lines). ALDA and PBE break down when the charge plasmon falls below the p-h line. . . . . . . . . . 23

2.5 Critical width $L_{\text {crit }}^{\text {inter }}$ at which the intersubband plasmon breakdown occurs, as a function of sheet density $N_{s}$. Full line: ALDA, dashed

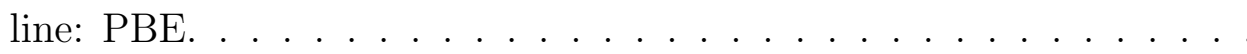

2.6 Plasmon dispersions $\Omega\left(q_{\|}\right)$for strictly 2D systems with sheet densities $N_{s}=10^{10}, 10^{11}$, and $10^{12} \mathrm{~cm}^{-2}$, calculated with RPA, 2D ALDA and PGG. The full lines denote the upper boundaries of the particle-hole (p-h) continuum. Here, $\tilde{q}_{\|}=q_{\|} / k_{F}^{2 \mathrm{D}}$ and $\tilde{\Omega}=\Omega /\left(k_{F}^{2 \mathrm{D}}\right)^{2} \ldots \ldots \ldots$

2.7 Intrasubband plasmon dispersions for quantum wells with sheet density $N_{s}=10^{10} \mathrm{~cm}^{-2}$, for different widths $L=\lambda L_{2}$, where $\lambda$ takes on the values $1,0.5,0.2,0.1,0.05,0.02,0.01,0.005,0.002$, and 0.001 . $L_{2}=217 \mathrm{~nm}$ is the largest width for which only the lowest subband is occupied. The individual plasmon dispersions are offset for clarity. The dashed lines are the upper boundaries of the p-h continuum. The squares indicate the wavevector $\tilde{q}_{\| p-h}$ where the plasmons enter the p-h continuum. Top panel: 3D ALDA. Bottom panel: PGG. . . . . 
2.8 Wavevector $\tilde{q}_{\mid p-h}$ at which the intrasubband plasmon merges with the p-h continuum, plotted versus well width scaling factor $\lambda$, calculated with PGG (blue) and ALDA (red). The dashed lines indicate the respective limits for the strictly $2 \mathrm{D}$ case. The calculations were done for sheet densities $N_{s}=10^{10}, 10^{11}, 10^{12}$, and $10^{13} \mathrm{~cm}^{-2}$, as indicated. The breakdown of the 3D ALDA occurs around $\lambda=0.1$ for all $N_{s}$. .

3.1 Reference frames $\mathcal{R}^{\prime}$ (black) and $\mathcal{R}$ (red) used to describe the electronic states in a quantum well with in-plane magnetic field $\mathbf{B}$ and spin-wave propagation direction q. . . . . . . . . . . . . . . 46

3.2 Spin-split lowest subband, Eq. (3.40), of an asymmetrically doped 20 nm CdTe quantum well with $B=4.18 \mathrm{~T}$, with $\alpha=2.2 \mathrm{meV \AA}$ and $\beta=3.9$ meVA, taken at an angle $\varphi=45^{\circ}$ (i.e. along [110]). The inset shows the quantum well profile and the electronic density distribution.

3.3 Spin-flip excitation spectra with $\operatorname{SOC}$ for $\varphi=45^{\circ}$ and $\varphi=135^{\circ}$, calculated using the ALDA for the same quantum well as in Fig. 3.2. Solid black lines: boundaries of the single-particle spin-flip continuum. Blue dashed lines: spin-wave dispersions. . . . . . . . . . . . . . .

3.4 (a) Electronic Raman scattering geometry: $\boldsymbol{k}_{i}$ and $\boldsymbol{k}_{s}$ are the incoming and scattered light wave vectors, respectively; $\mathbf{q}$ is the transferred momentum, of in-plane orientation measured by the angle $\varphi$ from [100]. An external magnetic field $\mathbf{B}_{\text {ext }}$ is applied perpendicularly to $\mathbf{q}$. (b) Raman spectra of the spin wave, obtained at $B_{\text {ext }}=2 \mathrm{~T}$ and $q=0$, for a series of in-plane angles $\varphi$. (c) Momentum dispersion of the spin wave for different in-plane angles. . . . . . . . . . . . . . . 
3.5 Coefficients $E_{0}, E_{1}$, and $E_{2}$ of the spin-wave dispersion, Eq. (3.4), as a function of angle $\varphi$. Dots: experimental data. Lines: theoretical results using $Z^{*}=0.573 \mathrm{meV}$ obtained with ALDA, and $\alpha=1.6$ meVA and $\beta=3.1$ meVA obtained by fitting $E_{0}$ and $E_{1}$. The red lines follow from the fully numerical solution of Eq. (3.60), the dashed blue lines follow from the analytical formulas (3.6) and (3.5). . . . .

3.6 Same as Fig. 3.5, but using $Z^{*}=0.63 \mathrm{meV}, \alpha=2.2 \mathrm{meV} \AA$, and $\beta=3.9$ meVA obtained from a best fit to the experimental data. . .

3.7 Amplitude of the modulation of the $q=0$ spin-wave energy, $\Delta E_{0}=$ $\left(\operatorname{Max} E_{0}-\operatorname{Min} E_{0}\right) / 2$, as a function of the sheet density $N_{s}$ of the electron gas in the quantum well. Black dots: experimental data. Blue line: analytical results using Eq. (3.6). . . . . . . . . . . . . . .

4.1 Reference frames $\mathcal{R}^{\prime}$ (black) and $\mathcal{R}$ (red) for the electronic states in a 2DEG with SOC and in-plane magnetic field $\mathbf{B}$. The striped pattern along the $\varphi=45^{\circ}$ direction indicates the persistent spin helix state of the 2DEG, with wave vector $\mathbf{Q}$, which forms in the absence of $\mathbf{B}$ if the Rashba and Dresselhaus coupling strengths are equal. . . . . . . . . 
4.2 Single-particle energies $E_{-, \mathbf{k}}$ and $E_{+, \mathbf{k}}$ for $\alpha=0.05$ and $\mathbf{k}$ along the [110] direction, see Eq. (4.13). (a) No magnetic field $\left(Z^{*}=0\right)$. Linear combinations of $E_{-, \mathbf{k}}$ and $E_{+, \mathbf{k}+\mathbf{Q}}$ states have spin helix texture, but these cancel out if summed over all occupied states below $E_{F}$. A persistent spin helix appears if a quasiparticle is injected at the Fermi surface, as shown. (b) Finite magnetic field $\left(Z^{*}=0.0381\right)$. Singleparticle excitations across the Fermi energy with momentum transfer Q (green arrow) give rise to propagating spin helices. . . . . . . . .

4.3 (a) Spin-wave dispersion $\omega_{\mathrm{sw}, 0}(\mathbf{q})$ (line) and single-particle spin-flip continuum (shaded area) without SOC. (b) Spin-wave dispersion $\omega_{\mathrm{sw}}(\mathbf{q})$ and single-particle spin-flip continuum, plotted along [110], for $\alpha=\beta=$ $0.003(Q=0.012)$. The 2DEG parameters are $r_{s}=2, \zeta=-0.0762$, $Z=0.02$, and $Z^{*}=0.0381$ (all values are in atomic units). The inset shows the position of Larmor's mode in the wave vector plane $\left(q_{x^{\prime}}, q_{y^{\prime}}\right) .84$

4.4 Proposed experimental design of the optical excitation and subsequent detection of the spin-helix Larmor mode using a grating coupler (see text for details). . . . . . . . . . . . . . . . . 86

4.5 Spin-wave stiffness of the 2DEG, obtained with the ALDA, for various values of the spin polarization $\zeta$ between 0.05 and 0.95 . . . . . . .

C.1 Photoabsorption cross section for $q_{\|}=0$ intersubband charge plasmons, for a quantum well with 5 occupied subbands, comparing PGG and exchange-only ISTLS. . . . . . . . . . . . . . . . . . 104 
E.1 Coefficients $E_{0}, E_{1}$, and $E_{2}$ of the spin-wave dispersion, Eq. (3.4), as a function of angle $\varphi$. Dots: experimental data. Lines: theoretical results with $g_{x y}=0.024$, using $Z^{*}=0.569 \mathrm{meV}$ obtained with ALDA, and $\alpha=1.4 \mathrm{meV} \AA$ and $\beta=3.0 \mathrm{meV} \AA$ obtained by fitting $E_{0}$ and $E_{1}$. The red lines follow from the fully numerical solution of Eq. (3.60), the dashed blue lines follow from the analytical formulas (3.6) and (3.5).118

E.2 Same as Fig. E.1, but using $Z^{*}=0.63 \mathrm{meV}, \alpha=2.1 \mathrm{meV} \AA$, and $\beta=3.5$ meVA obtained from a best fit to the experimental data. . . 119 


\begin{abstract}
Quasi-two-dimensional (2D) systems, such as an electron gas confined in a quantum well, are important model systems for many-body theories, and we are interested in studying collective excitations in such systems using a linear-response approach based on time-dependent density-functional theory.

In the second chapter, we consider a non-spin-polarized electron gas confined in a quantum well, and we study three- to two-dimensional crossover in time-dependent density-functional theory. Earlier studies of the crossover from 3D to 2D in groundstate density-functional theory showed that local and semilocal exchange-correlation functionals which are based on the 3D electron gas are appropriate for wide quantum wells, but eventually break down as the 2D limit is approached. We now consider the dynamical case and study the performance of various linear-response exchange kernels in time-dependent density-functional theory. We compare approximate local, semilocal, and orbital-dependent exchange kernels, and analyze their performance for inter- and intrasubband plasmons as the quantum wells approach the 2D limit. 3D (semi)local exchange functionals are found to fail for quantum well widths comparable to the 2D Wigner-Seitz radius $r_{s}^{2 D}$, which implies in practice that $3 \mathrm{D}$ local exchange remains valid in the quasi-2D dynamical regime for typical quantum well parameters, except for very low densities.

In the third chapter, we consider a partially spin-polarized electron gas in a semiconductor quantum well in the presence of Rashba and Dresselhaus spin-orbit coupling. Larmor's theorem holds for magnetic systems that are invariant under spin rotation. In the presence of spin-orbit coupling this invariance is lost and Larmor's
\end{abstract}


theorem is broken: for systems of interacting electrons, this gives rise to a subtle interplay between the spin-orbit coupling acting on individual single-particle states and Coulomb many-body effects. Using a linear-response approach based on timedependent density-functional theory in our system, we calculate the dispersions of spin-flip waves. We obtain analytic results for small wave vectors and up to second order in the Rashba and Dresselhaus coupling strengths $\alpha$ and $\beta$. Comparison with experimental data from inelastic light scattering allows us to extract $\alpha$ and $\beta$ as well as the spin-wave stiffness very accurately. We find significant deviations from the local density approximation for spin-dependent electron systems.

In the last chapter, we consider a two-dimensional electron gas (2DEG) with equal-strength Rashba and Dresselhaus spin-orbit coupling. This system sustains persistent helical spin-wave states, which have remarkably long lifetimes. In the presence of an in-plane magnetic field, there exist single-particle excitations that have the character of propagating helical spin waves. For magnon-like collective excitations, the spin-helix texture reemerges as a robust feature, giving rise to a decoupling of spin-orbit and electronic many-body effects. We prove that the resulting spin-flip wave dispersion is the same as in a magnetized 2DEG without spin-orbit coupling, apart from a shift by the spin-helix wave vector. The precessional mode about the persistent spin-helix state is shown to have an energy given by the bare Zeeman splitting, in analogy with Larmor's theorem. We also discuss ways to observe the spin-helix Larmor mode experimentally. 


\section{Chapter 1}

\section{Introduction}

In this thesis, we study the collective charge and spin excitations of itinerant electronic systems in quasi-two-dimensional semiconductor nanostructures. Our main focus is on the fundamental properties of a two-dimensional electron gas (2DEG) in a doped semiconductor quantum well because it is a paradigm of an electronic many-body system. The 2DEG has been thoroughly studied for many decades [1, 2], and it still is a subject of great fundamental and practical interest.

Let us begin by discussing some basic concepts of semiconductor quantum wells $[3,4]$. A semiconductor quantum well is illustrated on the left-hand side of Fig. 1.1,

where material $\mathrm{A}$, with a smaller band gap $E_{g}$, is sandwiched between two layers of material B (with a larger band gap). We only consider semiconductors with direct band gaps, such as GaAs, where the energy dispersions of the valence band and conduction band are aligned with maxima and minima, respectively, at the Brillouin zone center; electrons can then directly emit photons when undergoing interband transitions. 


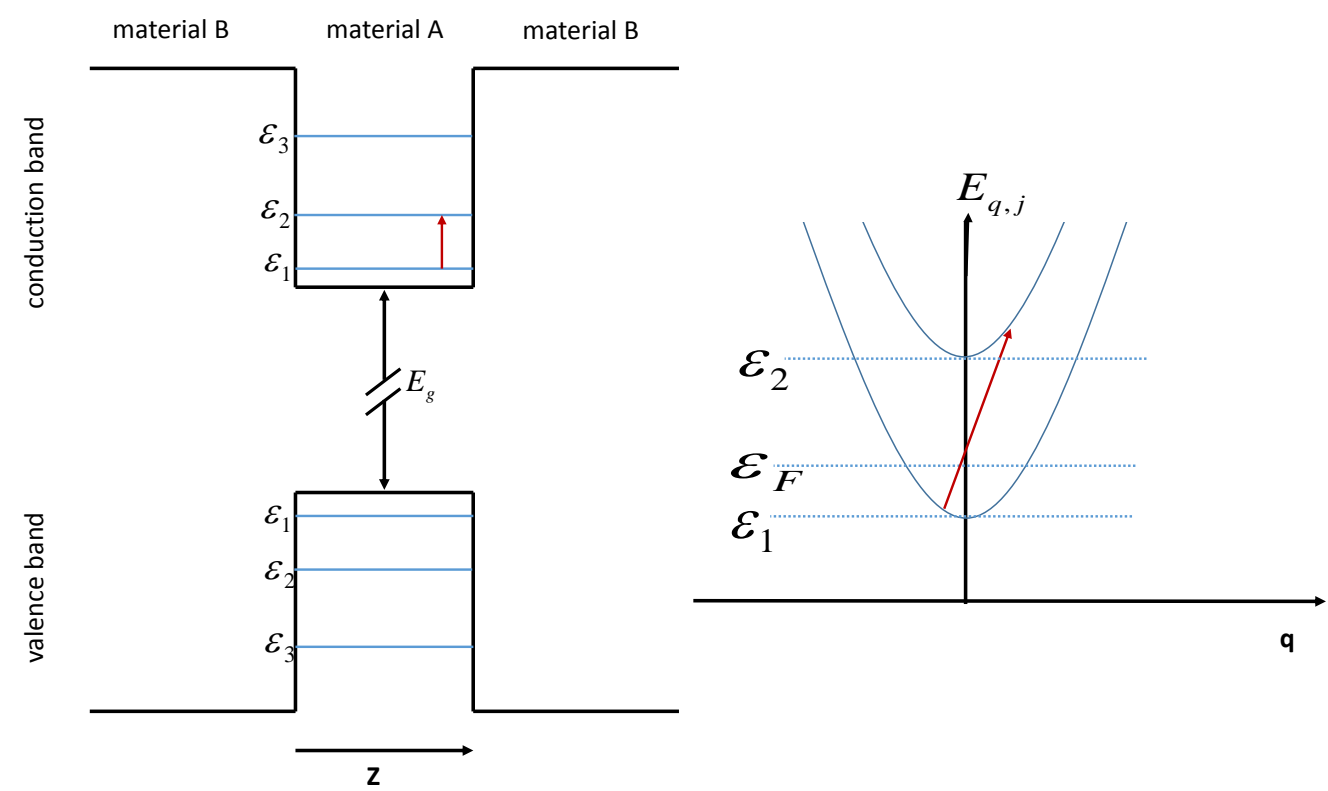

Figure 1.1: Left: Combining two semiconductor materials, A and B, with different band gaps, gives rise to a potential well in conduction and valence band. The electronic levels within the well are quantized into so-called subbands. Here, three subband levels $\varepsilon_{1}, \varepsilon_{2}$, and $\varepsilon_{3}$ are shown. Right: intersubband excitations where the first subband is occupied up to the conduction band Fermi level $\varepsilon_{F}$.

Here we are interested in $n$-doped systems where the electrons live in the conduction band of the quantum well (material A in Fig. 1.1). Since the electronic structure has a periodically-repeating environment, the electronic states, according to Bloch's theorem, have the form $\psi_{n \mathbf{q}}(\mathbf{r})=e^{i \mathbf{q r}} u_{n \mathbf{q}}(\mathbf{r})$, where $u_{n \mathbf{q}}(\mathbf{r})$ is a function with lattice periodicity. It can be proven that the electrons that are placed in the bottom of the conduction band of the crystalline semiconductor behave as free electrons, except that the electron mass must be replaced with the effective mass, $m^{*}$. Within the so-called effective mass approximation, the energy dispersion of the the conduction subbands are parabolic, see the right-hand side of Fig. 1.1. Transitions between different subbands are called intersubband transitions. 

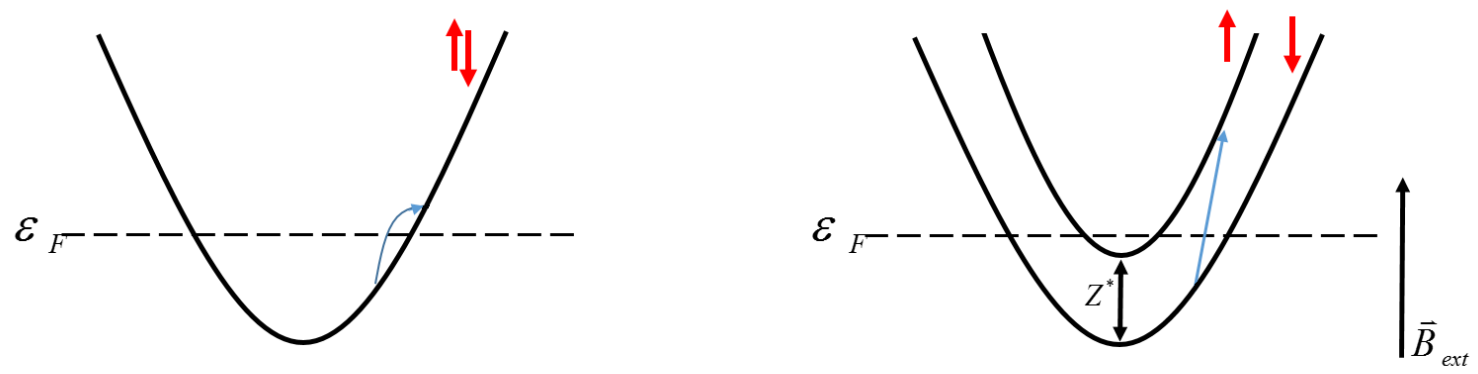

Figure 1.2: Left: Single-particle excitation of a 2DEG which is not spin polarized. The excitation is between states inside the Fermi circle $\varepsilon_{F}$ to states outside. Right: a 2DEG in the presence of a magnetic field, which lifts the spin degeneracy and splits the bands by the effective Zeeman energy $Z^{*}$. The thin blue arrow indicates a single-particle spin-flip transition.

If only the lowest subband is occupied, and the Fermi energy is not too high (so that the subband remains parabolic), we have the idealized situation of a 2DEG. The left part of Fig. 1.2 shows excitations in a conduction band where the lowest subband is occupied up to the Fermi level, and we have excitations from occupied states to unoccupied states within the first subband. The Fermi surface of a 2DEG is a circle, so these excitations are between filled states within the Fermi circle to states outside. We also refer to these excitations as intrasubband transitions.

In the absence of magnetic fields, spin-up and spin-down subbands are exactly on top of each other. An external in-plane magnetic field, $B_{\text {ext }}$, causes a Zeeman splitting of the subbands, where spin-up and spin-down subbands will be shifted apart by the effective Zeeman energy $Z^{*}$ which is proportional to an effective magnetic field, $B_{\text {eff }}$ As we will discuss in more detail in Chapter 3, the effective magnetic field, $B_{\text {eff }}=B_{\text {ext }}+B_{\mathrm{xc}}$, includes an exchange and correlation contribution $B_{\mathrm{xc}}$ caused by Coulomb many-body effects. In this case, we can distinguish between spin-conserving 
and spin-flip excitations. The example shown in Fig. 1.2 is of the spin-flip type.

In many semiconductor materials, spin-orbit coupling effects are playing an important role $[5,6]$. In recent years, spin-orbit coupling has attracted much interest in the context of spintronics [7], as well as in novel materials such as graphene [8] and topological insulators $[9,10,11]$. Spin-orbit coupling is present in all matter, and with various consequences for the electronic structure. Here, our interest is in those spin-orbit effects that are a consequence of the breaking of inversion symmetry of the system: the Dresselhaus and the Rashba effect. The Dresselhaus effect is related to the breaking of the crystalline inversion symmetry; the Rashba effect is caused by the breaking of symmetry in a structure or device, such as a quantum well in an electric field (which causes a linear potential).

Spin-orbit coupling is a relativistic effect, where moving electrons in motion experience electric fields as magnetic fields in their rest frame, which then interact with their spin. Hence, we can describe the effects of spin-orbit coupling in terms of effective crystal magnetic fields $\mathbf{B}_{\mathrm{SO}}(\mathbf{k})$, where $\mathbf{k}$ is the wavevector. In other words, Bloch electrons will experience different spin-orbit magnetic fields depending on their state of motion. Figure 1.3 indicates the symmetry of the Rashba and Dresselhaus fields, given, in a $2 \mathrm{DEG}$, by

$$
\mathbf{B}_{\mathrm{SO}}^{\text {Rashba }}(\mathbf{k})=\frac{2 \alpha}{g^{*} \mu_{B}}\left(\begin{array}{c}
k_{y} \\
-k_{x}
\end{array}\right), \quad \quad \mathbf{B}_{\mathrm{SO}}^{\text {Dresselhaus }}(\mathbf{k})=\frac{2 \beta}{g^{*} \mu_{B}}\left(\begin{array}{c}
k_{x} \\
-k_{y}
\end{array}\right) .
$$

Here, $\alpha$ and $\beta$ are the coupling strengths, $g^{*}$ is the effective $g$-factor, and $\mu_{B}$ is the Bohr magneton. As can be seen, the Rashba field has a vortex-like structure, whereas the Dresselhaus field is anti-vortex-like. 


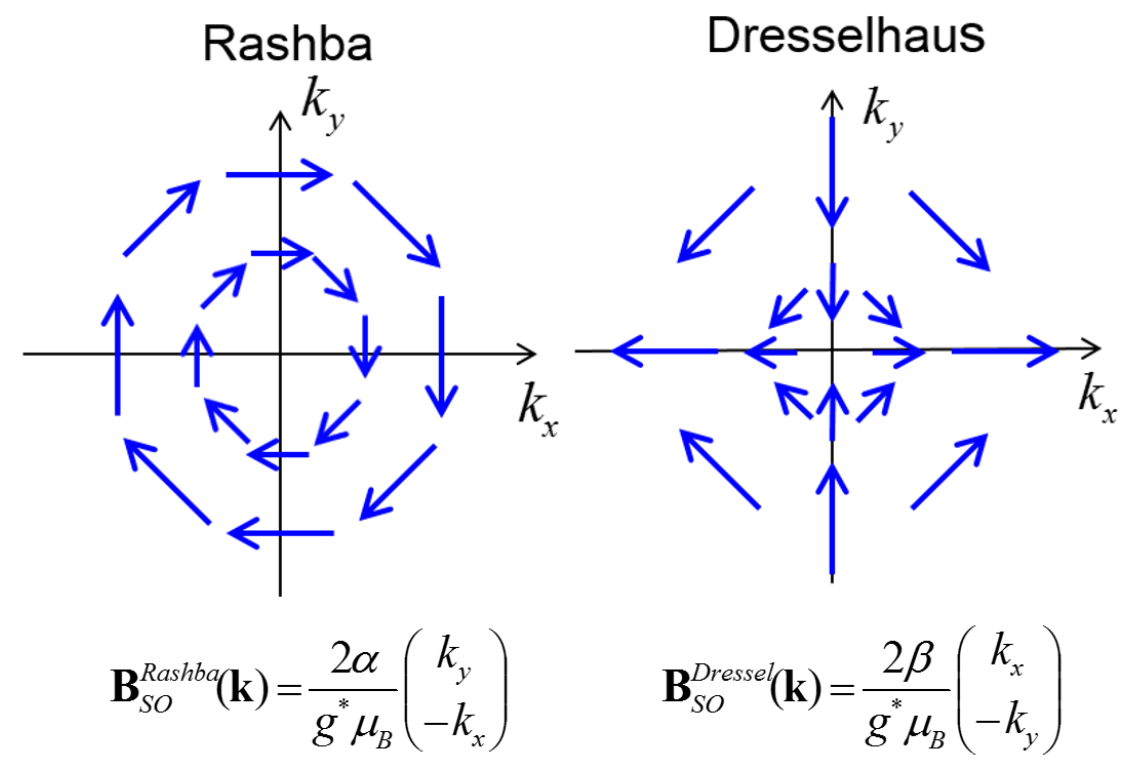

Figure 1.3: Symmetry of the Rashba and Dresselhaus spin-orbit effective magnetic fields. Note that if both effects are present, the net spin-orbit field strength has elliptic symmetry.

The central theme of this thesis will be those situations where Coulomb interactions have important consequences, causing new forms of collective behavior. Most notably, Coulomb interactions are responsible for the formation of plasmons. Another example is spin waves in the spin-polarized 2DEG, which can be thought of as a collective spin precession propagating through the system [12]. Electronic spin waves are the itinerant-electron counterpart of magnons, which are collective precessions in lattices of localized spins. Magnon spintronics [13, 14], a new and promising subfield of spintronics, is based on the idea that information can be encoded and transported by spin waves. The spin-orbit interaction plays an important role in magnon spintronics since it provides a coupling mechanism between spin dynamics and electrical signals $[15,16]$. However, so far, the interplay between spin-orbit coupling and Coulomb many-body effects has been relatively little explored. 
In this thesis, we will investigate the following questions:

- What happens when a system of electrons in a quantum well becomes more and more confined, so that it goes through a cross-over between a $3 \mathrm{D}$ to a $2 \mathrm{D}$ state [17]? In particular, can we find theoretical methods to calculate plasmon modes in the system that are robust under such a cross-over? We will answer these questions in Chapter 2.

- How are collective spin-wave excitations in a spin-polarized 2DEG affected by the presence of Rashba and Dresselhaus spin-orbit interactions [18]? Spin waves are well-ordered collective modes of the electron system; will their order be destroyed by the spin-orbit effects, or will it be modified in more subtle ways? How do our theoretical methods hold up under comparison with experimental results? We will study this in Chapter 3.

- There is an intriguing special situation in which the Rashba and Dresselhaus fields have the same strengths, $\alpha=\beta$. From the recent literature, it is known that this leads to so-called spin-helical states. What happens to collective spin waves in this situation? The answer will be given in Chapter 4, including a proposal for an experimental setup to observe a new exact dynamical manybody state which we call the spin-helix Larmor mode.

Each Chapter is self-contained, with additional technical details given in several Appendices. However, several cross-references and connections between the Chapters will be pointed out. In the final Chapter 5, a summary and an outlook are given. 


\section{Chapter 2}

\section{Three- to two-dimensional crossover in time-dependent density-functional theory}

The key concept of density-functional theory (DFT) [19] is that all electronic manybody systems can be uniquely characterized by their electron density $n(\mathbf{r})$. The density can be obtained in principle exactly via the Kohn-Sham equation (here and in the following we use atomic units) [20],

$$
\left[-\frac{\nabla^{2}}{2}+v_{0}(\mathbf{r})+v_{\mathrm{H}}[n](\mathbf{r})+v_{\mathrm{xc}}[n](\mathbf{r})\right] \varphi_{j}(\mathbf{r})=\varepsilon_{j} \varphi_{j}(\mathbf{r}),
$$

where $v_{0}(\mathbf{r})$ is a given external potential, $v_{\mathrm{H}}[n](\mathbf{r})=\int d^{3} r^{\prime} n\left(\mathbf{r}^{\prime}\right) /\left|\mathbf{r}-\mathbf{r}^{\prime}\right|$ is the Hartree potential, and $v_{\mathrm{xc}}[n](\mathbf{r})$ is the exchange-correlation (xc) potential. The density is obtained from the self-consistent solution of Eq. (2.1) as $n(\mathbf{r})=\sum_{j=1}^{N}\left|\varphi_{j}(\mathbf{r})\right|^{2}$, where $N$ is the number of electrons, and all physical observables follow therefrom.

The xc potential is defined as the functional derivative $v_{\mathrm{xc}}[n](\mathbf{r})=\delta E_{\mathrm{xc}}[n] / \delta n(\mathbf{r})$. 
The xc energy $E_{\mathrm{xc}}[n]$ is a universal functional of the density: this means that there is one and only one exact density functional of the xc energy that is valid for all electronic systems with a given form of the electron-electron interaction, for any $N$. If this exact xc functional were known, it would give exact ground-state results, via Eq. (2.1), for all conceivable forms of matter, including atoms, molecules, and periodic or non-periodic solids.

In real matter, $v_{0}(\mathbf{r})$ consists of the Coulomb potentials of positively charged atomic nuclei. But the universality of $E_{\mathrm{xc}}[n]$ and $v_{\mathrm{xc}}[n](\mathbf{r})$ extends beyond real matter, and includes all mathematically reasonable forms of $v_{0}(\mathbf{r})$, whether they exist in nature or not. In particular, it includes systems of lower dimensionality, for instance electrons confined in a two-dimensional (2D) plane [21].

A stringent test for approximate $\mathrm{xc}$ functionals is their performance during a dimensional crossover. The crossover from $3 \mathrm{D}$ to $2 \mathrm{D}$ has been previously studied in the DFT literature $[22,23,24,25]$. It was found that local and semilocal functionals such as the local-density approximation (LDA) and generalized gradient approximations (GGAs) fail badly at this task. To see this, consider the LDA exchange energy

$$
E_{\mathrm{x}, 3 \mathrm{D}}^{\mathrm{LDA}}[n]=-\frac{3}{4}\left(\frac{3}{\pi}\right)^{1 / 3} \int d^{3} r n(\mathbf{r})^{4 / 3} .
$$

What happens if we try to evaluate $E_{\mathrm{x}, 3 \mathrm{D}}^{\mathrm{LDA}}[n]$ for a $2 \mathrm{D}$ system? Let the density be $n_{2 \mathrm{D}}(\mathbf{r})=n\left(\mathbf{r}_{\|}\right) \delta(z)$, where $\mathbf{r}_{\|}=(x, y)$ denotes a $2 \mathrm{D}$ position vector. Using the delta function in the form $\delta(z)=\lim _{\epsilon \rightarrow 0^{+}}(4 \pi \epsilon)^{-1 / 2} e^{-z^{2} / 4 \epsilon}$, one finds

$$
E_{\mathrm{x}, 3 \mathrm{D}}^{\mathrm{LDA}}\left[n_{2 \mathrm{D}}\right]=\lim _{\epsilon \rightarrow 0^{+}} \frac{3^{11 / 6}}{4^{5 / 3} \sqrt{\pi} \epsilon^{1 / 6}} \int d^{2} r_{\|} n\left(\mathbf{r}_{\|}\right)^{4 / 3}
$$


This clearly shows that the 3D form of the LDA exchange energy diverges in the $2 \mathrm{D}$ limit, instead of approaching the proper form of the 2D LDA [2],

$$
E_{\mathrm{x}, 2 \mathrm{D}}^{\mathrm{LDA}}[n]=-\frac{4}{3} \sqrt{\frac{2}{\pi}} \int d^{2} r_{\|} n\left(\mathbf{r}_{\|}\right)^{3 / 2}
$$

All standard 3D GGAs will exhibit a similar divergence in the 2D limit.

To capture the 3D-2D crossover correctly, nonlocal xc functionals are needed. Some improvement over LDA and GGAs can be achieved with meta-GGA and hyperGGA xc functionals $[24,25]$, but only fully nonlocal xc functionals such as the average density approximation [23] or the inhomogeneous STLS [26, 27] show a proper behavior as the 2D limit is approached.

Here we extend the study of the dimensional crossover into the domain of timedependent density-functional theory (TDDFT) $[28,29,30]$. However, we will not explore the full dynamical range of TDDFT, which allows one to study electronic systems under the influence of arbitrary external time-dependent potentials, $v(\mathbf{r}, t)$; instead, we will limit ourselves to the linear-response regime and consider electronic excitation energies [31, 32]. Furthermore, in this chapter we will only consider exchange, but not correlation effects.

The main questions are the following. What characteristic effects or signatures occur in the excitation spectrum of a system as it crosses over from three to two dimensions, and how will the expected failure of LDA and GGA manifest itself? Will the breakdown be as drastic as in ground-state DFT, or will it perhaps be less severe, under some circumstances? How do nonlocal orbital functionals perform under the 3D-2D crossover?

Apart from the inherent fundamental interest, there are important practical rea- 

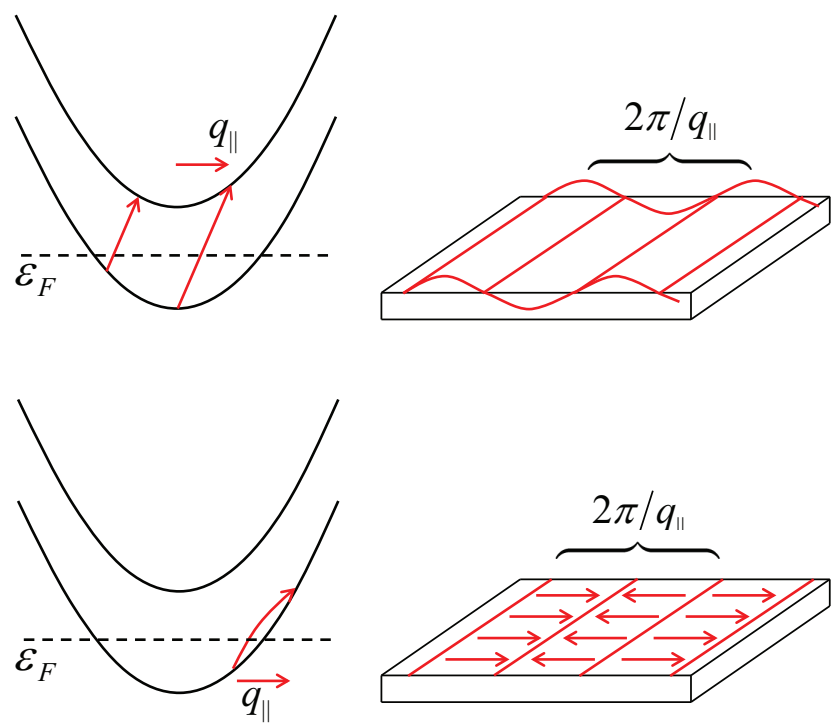

Figure 2.1: Illustrations of intersubband (top) and intrasubband (bottom) plasmon excitations with wavevector $q_{\|}$in a quantum well with conduction band Fermi level $\varepsilon_{F}$ in the lowest subband. Intersubband plasmons involve collective transitions between two subbands, leading to density oscillations of the quasi-2D electron system perpendicular to the quantum well plane. Intrasubband plasmons (collective transitions within the lowest subband) are characterized by density oscillations and currents flowing along the plane.

sons that motivate such a study. Quasi-2D [33] electron gases (2DEGs) can be prepared in very high quality along interfaces and in heterostructures of a wide range of materials (most notably semiconductors and oxides), with many practical applications $[3,4]$. It is important to be able to model the electronic structure and dynamics in these systems accurately and numerically efficiently. Since no DFT method beats the LDA in terms of simplicity and efficiency, one would like to know whether the 3D LDA is reliable in the quasi-2D regime, and under what circumstances it starts to fail. We will answer these questions in the following.

Figure 2.1 illustrates the two types of collective excitations that we will study in 
this chapter. In a quantum well, electrons are free to move in the plane, but the levels are quantized into subbands due to quantum confinement perpendicular to the plane. Intersubband plasmons involve transitions from occupied to empty subbands; since different subbands have different envelope functions, this implies density oscillations perpendicular to the well plane. By contrast, intrasubband plasmons involve transitions within a subband; the accompanying currents and density oscillations are parallel to the plane. We will study what happens to these excitations as the quantum well becomes more and more narrow, approaching the strictly 2D limit.

This chapter is organized as follows. In Section 2.1 we discuss the necessary theoretical background: we introduce our quantum well model, review the TDDFT linear-response formalism for collective excitations in quantum wells, and list various exchange functionals. In Section 2.2 we present our results, and Section 2.3 gives conclusions. Some technical details are given in the Appendix.

\subsection{Theoretical background}

\subsubsection{Quantum well model}

We consider n-doped semiconductor quantum wells of width $L$ in which the electrons are confined along the $z$ direction and free to move in the $x-y$ plane. The number of electrons per unit area (the sheet density) is denoted by $N_{s}$. In the following, we assume that the material of the quantum well is GaAs, with effective mass $m^{*}=$ $0.067 m$ and effective charge $e^{*}=e / \sqrt{13}(m$ and $e$ are the free electron mass and charge). We choose units in which $e^{*}=m^{*}=\hbar=1$. The effective Hartree unit of 
energy is $10.8 \mathrm{meV}$; the effective Bohr radius is $103 \AA$.

The quantum well is assumed to be confined within infinitely high barriers at $z=0$ and $z=L$. We further assume that the solutions of the Kohn-Sham equation for the quantum well envelope functions $[3,4]$ have the standard particle-in-a-box form,

$$
\varphi_{j}(z)=\sqrt{\frac{2}{L}} \sin \left(\frac{j \pi z}{L}\right), \quad j=1,2,3, \ldots,
$$

with Kohn-Sham energies

$$
\varepsilon_{j}=\frac{1}{2}\left(\frac{j \pi}{L}\right)^{2}
$$

The Kohn-Sham potential $v_{s}(z)=v_{\mathrm{ext}}(z)+v_{\mathrm{H}}(z)+v_{\mathrm{xc}}(z)$ that gives rise to these solutions is an infinitely deep square-well potential. This means that for each $L$ and $N_{s}$ the external quantum well potential $v_{\text {ext }}(z)$ is chosen such that, if added to the Hartree and xc potentials $v_{\mathrm{H}}(z)$ and $v_{\mathrm{xc}}(z)$, the resulting sum is a constant for $0<z<L$. Thanks to the Hohenberg-Kohn theorem [19], a unique choice of such a $v_{\text {ext }}(z)$ is always possible in principle; further details of the ground-state potentials do not need to be specified in the following.

We emphasize that the particle-in-a-box form of the Kohn-Sham eigenstates is only a matter of convenience, and does not lead to a loss of generality of the results of the 3D-2D crossover that we study here.

The ground-state density in the well is given by

$$
n_{0}(z)=\frac{1}{\pi} \sum_{\substack{j \\ \varepsilon_{j}<\varepsilon_{F}}} \varphi_{j}^{2}(z)\left(\varepsilon_{F}-\varepsilon_{j}\right),
$$

where the factor $\left(\varepsilon_{F}-\varepsilon_{j}\right) / \pi$ comes from the summation of all occupied single-particle 
states in the $j$ th subband. To determine the Fermi energy $\varepsilon_{F}$, we integrate the density over $z$ :

$$
\int_{0}^{L} d z n_{0}(z)=N_{s}=\frac{1}{\pi} \sum_{j=1}^{N_{\text {occ }}}\left(\varepsilon_{F}-\varepsilon_{j}\right)
$$

where $N_{\text {occ }}$ is the number of occupied subbands. Hence,

$$
\varepsilon_{F}=\frac{\pi N_{s}}{N_{\mathrm{occ}}}+\frac{1}{N_{\mathrm{occ}}} \sum_{j=1}^{\mathrm{N}_{\mathrm{occ}}} \varepsilon_{j}
$$

and $N_{\text {occ }}$ is fixed by requiring $\varepsilon_{N_{\text {occ }}}<\varepsilon_{F}<\varepsilon_{N_{\text {occ }}+1}$.

\subsubsection{Excitations within linear-response TDDFT}

In the following, we are interested in the frequency-dependent spin-density response in a quantum well. Because of the translational symmetry in the $x-y$ plane, we Fourier transform with respect to the in-plane position vector $\mathbf{r}_{\|}=(x, y)$; this introduces the in-plane wavevector $\mathbf{q}_{\|}$. The TDDFT linear-response equation [34] then becomes

$$
n_{1 \sigma}\left(\mathbf{q}_{\|}, z, \omega\right)=\int d z^{\prime} \chi_{s \sigma \sigma}\left(\mathbf{q}_{\|}, z, z^{\prime}, \omega\right) v_{s 1 \sigma}\left(\mathbf{q}_{\|}, z^{\prime}, \omega\right)
$$

The noninteracting response function is diagonal in the spin $\sigma$ :

$$
\chi_{s, \sigma \sigma^{\prime}}\left(\mathbf{q}_{\|}, z, z^{\prime}, \omega\right)=\delta_{\sigma \sigma^{\prime}} \sum_{j=1}^{N_{\text {occ }}} \sum_{l=1}^{\infty} F_{l j}\left(\mathbf{q}_{\|}, \omega\right) \varphi_{j}(z) \varphi_{l}(z) \varphi_{j}\left(z^{\prime}\right) \varphi_{l}\left(z^{\prime}\right),
$$


where

$$
\begin{aligned}
F_{l j}\left(\mathbf{q}_{\|}, \omega\right)= & \int \frac{d^{2} k_{\|}}{(2 \pi)^{2}}\left[\frac{\theta\left(\varepsilon_{F}-\varepsilon_{j}-k_{\|}^{2} / 2\right)}{\omega-\omega_{l j}-\mathbf{q}_{\|} \mathbf{k}_{\|}-q_{\|}^{2} / 2+i \eta}\right. \\
& \left.-\frac{\theta\left(\varepsilon_{F}-\varepsilon_{j}-k_{\|}^{2} / 2\right)}{\omega+\omega_{l j}+\mathbf{q}_{\|} \mathbf{k}_{\|}+q_{\|}^{2} / 2+i \eta}\right] .
\end{aligned}
$$

Here, $\omega_{l j}=\varepsilon_{l}-\varepsilon_{j}$, and $\eta$ is a positive infinitesimal. The linearized effective potential, $v_{s 1 \sigma}=v_{1 \sigma}+v_{\mathrm{Hxc} 1 \sigma}$, consists of an external scalar perturbation plus a linearized Hartree-xc contribution:

$$
v_{\mathrm{Hxc} 1 \sigma}\left(\mathbf{q}_{\|}, z, \omega\right)=\sum_{\sigma^{\prime}} \int d z^{\prime}\left[\frac{2 \pi}{q_{\|}} e^{-q_{\|}\left|z-z^{\prime}\right|}+f_{\mathrm{xc}, \sigma \sigma^{\prime}}\left(\mathbf{q}_{\|}, z, z^{\prime}, \omega\right)\right] n_{1 \sigma^{\prime}}\left(\mathbf{q}_{\|}, z^{\prime}, \omega\right)
$$

The xc kernel $f_{\mathrm{xc}, \sigma \sigma^{\prime}}$ will be discussed in more detail below.

The following external perturbation triggers both single-particle and collective excitations with a finite in-plane wave vector $\mathbf{q}_{\|}$:

$$
v_{1 \sigma}\left(\mathbf{q}_{\|}, z, \omega\right)=S_{\sigma}^{ \pm} E_{0} e^{q_{\|} z}
$$

which couples to the charge $(+)$ and the spin $(-)$ channel via $S_{\sigma}^{ \pm}=\delta_{\sigma, \uparrow} \pm \delta_{\sigma, \downarrow}$, respectively. Having solved the response equation (2.10) self-consistently, we obtain the absorption cross section [35] as

$$
\sigma\left(\mathbf{q}_{\|}, \omega\right)=-\frac{2 \omega}{E_{0} q_{\|}^{2}} \Im \sum_{\sigma} S_{\sigma}^{ \pm} \int d z e^{q_{||} z} n_{1 \sigma}\left(\mathbf{q}_{\|}, z, \omega\right)
$$

This expression can be viewed as a generalization of the so-called reflection amplitude, which determines the infrared absorption of quantum wells in the presence of a grating 
coupler $[36,37]$.

The absorption cross section (2.14), when plotted as a function of frequency, has peaks at those frequencies that are resonant with an excitation energy of the system; the peak height is a measure of the oscillator strength.

The alternative to calculating the absorption cross section is to directly calculate the excitation energies of the system. The idea is that an electronic excitation can be viewed as an electronic eigenmode, i.e., a dynamical response of the system that is self-sustained and does not require an external perturbation. The characteristic eigenmode frequencies are thus obtained as those frequencies $\Omega$ where the linearresponse equation has a nontrivial solution in the absence of an external perturbation $[30,31]$. The resulting general formalism for calculating excitation energies in TDDFT has the form of an eigenvalue equation [29, 32]:

$$
\left(\begin{array}{cc}
\mathrm{A} & \mathrm{K} \\
\mathrm{K} & \mathrm{A}
\end{array}\right)\left(\begin{array}{c}
\mathrm{X} \\
\mathrm{Y}
\end{array}\right)=\Omega\left(\begin{array}{cc}
-1 & 0 \\
0 & 1
\end{array}\right)\left(\begin{array}{l}
\mathrm{X} \\
\mathrm{Y}
\end{array}\right)
$$

where the matrix elements of $\mathbf{A}$ and $\mathbf{K}$ are given by

$$
\begin{gathered}
A_{i a \sigma, i^{\prime} a^{\prime} \sigma^{\prime}}(\omega)=\delta_{i i^{\prime}} \delta_{a a^{\prime}} \delta_{\sigma \sigma^{\prime}} \omega_{a i \sigma}+K_{i a \sigma, i^{\prime} a^{\prime} \sigma^{\prime}}(\omega) \\
K_{i a \sigma, i^{\prime} a^{\prime} \sigma^{\prime}}(\omega)=\int d^{3} r \int d^{3} r^{\prime} \varphi_{i}^{*}(\mathbf{r}) \varphi_{a}(\mathbf{r})\left\{\frac{1}{\left|\mathbf{r}-\mathbf{r}^{\prime}\right|}+f_{\mathrm{xc} \sigma \sigma^{\prime}}\left(\mathbf{r}, \mathbf{r}^{\prime}, \omega\right)\right\} \varphi_{i^{\prime}}\left(\mathbf{r}^{\prime}\right) \varphi_{a^{\prime}}^{*}\left(\mathbf{r}^{\prime}\right)
\end{gathered}
$$

and $i, i^{\prime}$ and $a, a^{\prime}$ run over occupied and unoccupied Kohn-Sham orbitals, respectively. In almost all applications of this formalism one uses frequency-independent approximations for the xc kernel. 
Equation (2.15) can be adapted in a rather straightforward manner to calculate inter- and intrasubband charge and spin plasmon frequencies in quantum wells; all one needs to do is use the explicit form $\varphi_{j}(\mathbf{r})=A^{-1 / 2} \varphi_{j}(z) e^{i \mathbf{k}_{||} \cdot \mathbf{r}_{\| \mid}}$of the single-particle wave functions and then Fourier transform with respect to $\mathbf{r}_{\|}$.

Rather than giving the general formalism, let us consider the much simpler (but very important) quasi-2D case. Assume that only the lowest subband is occupied, and consider the lowest intersubband plasmon modes at wavevector $\mathbf{q}_{\|}=0$. Ignoring the influence of the third and higher subbands, the intersubband charge and spin plasmon frequencies are given by

$$
\Omega_{c, s}^{2}=\omega_{21}^{2}+\omega_{21} N_{s}\left(K_{\uparrow \uparrow} \pm K_{\uparrow \downarrow}\right)
$$

where $\omega_{21}=\varepsilon_{2}-\varepsilon_{1}$ and

$$
K_{\sigma \sigma^{\prime}}=\int d z \int d z^{\prime} \varphi_{1}(z) \varphi_{2}(z)\left[-2 \pi\left|z-z^{\prime}\right|+f_{\mathrm{xc}, \sigma \sigma^{\prime}}\left(z, z^{\prime}\right)\right] \varphi_{1}\left(z^{\prime}\right) \varphi_{2}\left(z^{\prime}\right)
$$

For finite wavevectors, analytic expressions for the plasmon frequencies can be obtained for small values of $q_{\|}$by Taylor expansion of the response function; however, it is easier to determine the plasmon frequencies numerically.

\subsubsection{Exchange kernels}

The main purpose of this chapter is to compare the performance of different approximate xc kernels in the crossover from 3D to 2D. In the following we shall limit ourselves to the exchange-only case. For systems that are not spin polarized, the spin-resolved exchange kernel $f_{\mathrm{x}, \sigma \sigma^{\prime}}$ is obtained from the spin-unresolved exchange 
kernel $f_{\mathrm{x}}$ as

$$
f_{\mathrm{x}, \sigma \sigma^{\prime}}=2 \delta_{\sigma \sigma^{\prime}} f_{\mathrm{x}} .
$$

We compare three different frequency-independent exchange kernels: ALDA, PBE, and PGG. The ALDA exchange kernel is defined as follows:

$$
f_{\mathrm{x}}^{\mathrm{ALDA}}\left(\mathbf{r}, \mathbf{r}^{\prime}\right)=\left.\frac{d^{2} e_{\mathrm{x}}^{h}(\bar{n})}{d \bar{n}^{2}}\right|_{\bar{n}=n(\mathbf{r})} \delta\left(\mathbf{r}-\mathbf{r}^{\prime}\right),
$$

where $e_{\mathrm{x}}^{h}(n)$ is the exchange energy density of a homogeneous electron liquid of density $n$.[2] Hence, the 3D and 2D ALDA exchange kernels are given by

$$
\begin{aligned}
f_{\mathrm{x}, 3 \mathrm{D}}^{\mathrm{ALDA}}\left(\mathbf{r}, \mathbf{r}^{\prime}\right) & =-\left[9 \pi n^{2}(\mathbf{r})\right]^{-1 / 3} \delta\left(\mathbf{r}-\mathbf{r}^{\prime}\right) \\
f_{\mathrm{x}, 2 \mathrm{D}}^{\mathrm{ALDA}}\left(\mathbf{r}_{\|}, \mathbf{r}_{\|}^{\prime}\right) & =-\left[\pi n_{2 \mathrm{D}}\left(\mathbf{r}_{\|}\right) / 2\right]^{-1 / 2} \delta\left(\mathbf{r}_{\|}-\mathbf{r}_{\|}^{\prime}\right)
\end{aligned}
$$

The PBE functional [38] is probably the most widely used GGA; it is defined only for 3D systems. The explicit expression for the PBE exchange kernel turns out to be quite lengthy, and is given in Appendix A.

In contrast with ALDA and PBE, the so-called PGG functional [31, 39] is a nonlocal orbital functional, given by

$$
f_{\mathrm{x}}^{\mathrm{PGG}}\left(\mathbf{r}, \mathbf{r}^{\prime}\right)=-2 \frac{\left|\sum_{j=1}^{N_{\text {occ }}} \varphi_{j}(\mathbf{r}) \varphi_{j}^{*}\left(\mathbf{r}^{\prime}\right)\right|^{2}}{\left|\mathbf{r}-\mathbf{r}^{\prime}\right| n(\mathbf{r}) n\left(\mathbf{r}^{\prime}\right)},
$$

where the sum runs over $N_{\text {occ }}$ doubly occupied orbitals. PGG can be viewed as an approximation to the exact exchange kernel [29]. We give the explicit form of the PGG kernel for quasi-2D systems and for the 2D limit in Appendix B, and discuss 
its relation to exchange-only ISTLS in Appendix C.

\subsection{Results and Discussions}

\subsubsection{Plasmons: from bulk to quasi-2D}

Plasmons in homogeneous electron liquids have been thoroughly studied for many

decades [40]. The plasmon dispersions in 2D and 3D follow from the exact conditions

$$
\begin{aligned}
{\left[\frac{4 \pi}{q^{2}}+f_{\mathrm{xc}, 3 \mathrm{D}}\left(q, \Omega_{3 \mathrm{D}}\right)\right] \chi_{0}^{3 \mathrm{D}}\left(q, \Omega_{3 \mathrm{D}}\right) } & =1 \\
{\left[\frac{2 \pi}{q_{\|}}+f_{\mathrm{xc}, 2 \mathrm{D}}\left(q_{\|}, \Omega_{2 \mathrm{D}}\right)\right] \chi_{0}^{2 \mathrm{D}}\left(q_{\|}, \Omega_{2 \mathrm{D}}\right) } & =1,
\end{aligned}
$$

where $\chi_{0}^{3 \mathrm{D}}(q, \Omega)$ and $\chi_{0}^{2 \mathrm{D}}\left(q_{\|}, \Omega\right)$ are the 3D and 2D Lindhard functions [2]. In the limit of small wavevectors, one obtains

$$
\Omega_{3 \mathrm{D}}(q \rightarrow 0)=\omega_{\mathrm{pl}}\left[1+\left(\frac{3\left(k_{F}^{3 \mathrm{D}}\right)^{2}}{10 \omega_{\mathrm{pl}}^{2}}+\frac{f_{\mathrm{xc}, 3 \mathrm{D}}\left(0, \omega_{\mathrm{pl}}\right)}{8 \pi}\right) q^{2}\right]
$$

where $\omega_{\mathrm{pl}}=\sqrt{4 \pi n}$ is the classical plasma frequency of a 3D electron liquid of density $n$, and $k_{F}^{3 \mathrm{D}}$ is the associated Fermi wavevector. The corresponding relation in 2D is

$$
\Omega_{2 \mathrm{D}}\left(q_{\|} \rightarrow 0\right)=k_{F}^{2 \mathrm{D}} \sqrt{q_{\|}}\left[1+\frac{q_{\|}}{2 \pi} f_{\mathrm{xc}, 2 \mathrm{D}}(0,0)\right]^{1 / 2} .
$$

$\Omega_{3 \mathrm{D}}(q)$ and $\Omega_{2 \mathrm{D}}\left(q_{\|}\right)$both describe charge plasmons (i.e., collective oscillations of the charge density $n$ ). There are no corresponding 3D and 2D spin plasmons (i.e., collective oscillations of the spin density) as long as the system is not magnetic: the 
conditions for spin plasmons in homogeneous systems, $f_{\mathrm{xc}, 3 \mathrm{D}}\left(q, \Omega_{s}\right) \chi_{0}^{3 \mathrm{D}}\left(q, \Omega_{s}\right)=1$ and $f_{\mathrm{xc}, 2 \mathrm{D}}\left(q_{\|}, \Omega_{s}\right) \chi_{0}^{2 \mathrm{D}}\left(q_{\|}, \Omega_{s}\right)=1$, cannot be satisfied, i.e., there is no real or complex frequency $\Omega_{s}$ which makes the left-hand side equal to one.

Suppose now that we start from a homogeneous 3D system and let one of its dimensions, say $z$, become confined: this defines a neutral jellium slab [36, 37]. Let us consider a jellium slab that corresponds to the quantum well model with hard boundaries that we described in Section 2.1.1. What happens to the plasmon mode as the width $L$ of this system shrinks down to the quantum limit?

As soon as $L$ becomes finite, the collective excitations are described using the formalism of intersubband plasmons. We consider the case where the average 3D density $\bar{n}$ in the well is constant, letting

$$
\bar{n}=N_{s} / L
$$

If $L$ is very large, the difference between two consecutive energy levels $\varepsilon_{j}$ and $\varepsilon_{j+1}$, see Eq. (2.6), is very small, and a large number of subbands is occupied. As $L$ shrinks, the level spacing increases and fewer and fewer subbands are occupied. Let $L_{\nu}$ be that width where the Fermi energy $\varepsilon_{F}$ coincides with the $\nu$ th level $\varepsilon_{\nu}$. From Eqs. (2.6) and (2.9) it is straightforward to show that

$$
L_{\nu}^{3}=\frac{\pi \nu}{12 \bar{n}}\left(4 \nu^{2}-3 \nu-1\right),
$$

where we used $\sum_{j=1}^{\nu} j^{2}=\nu(\nu+1)(2 \nu+1) / 6$. In particular, for $\nu=2$ we have

$$
L_{2}=\left(\frac{3 \pi}{2 \bar{n}}\right)^{1 / 3}
$$



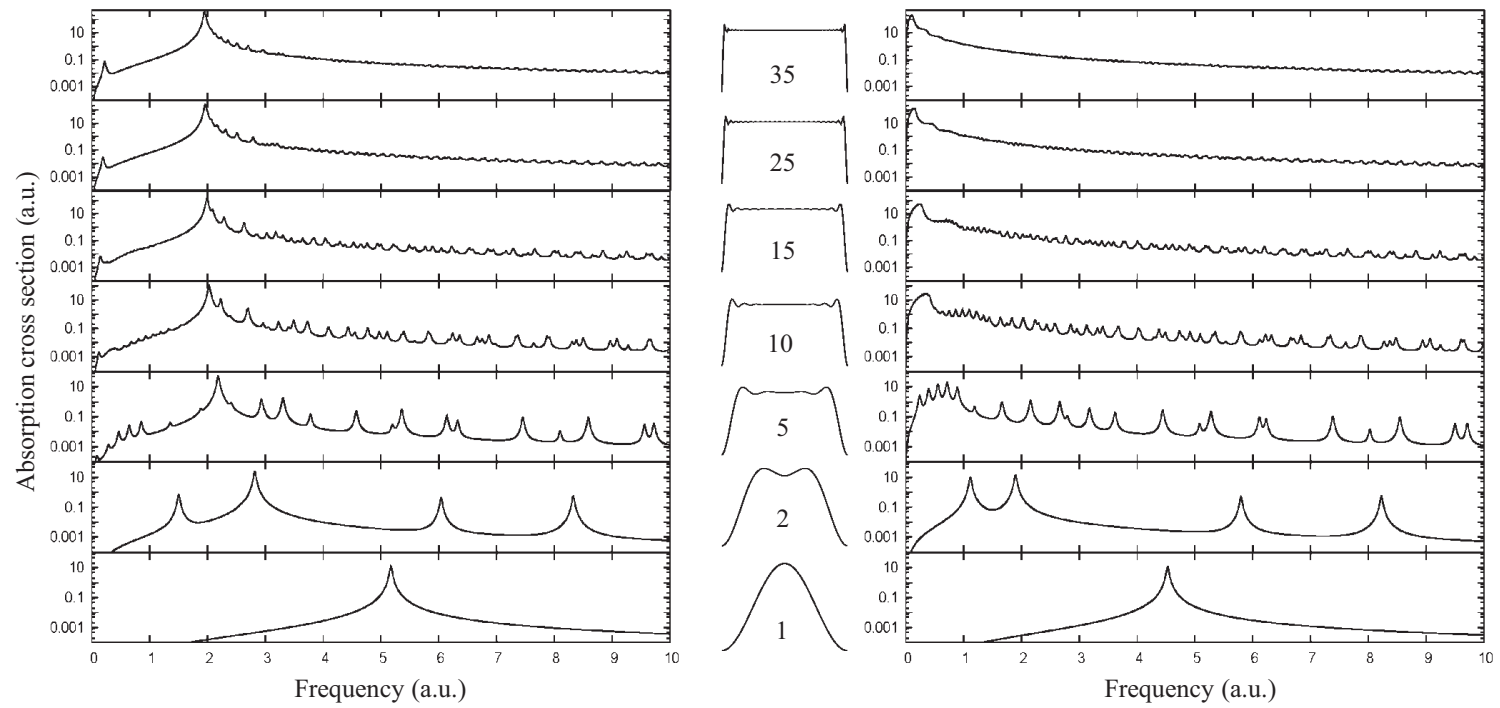

Figure 2.2: Photoabsorption cross section for $q_{\|}=0$ intersubband excitations in quantum wells. Left panels: charge-density excitations. Right panels: spin-density excitations. Insets: density profiles at given values of $N_{\text {occ }}$. The calculations were done with Eq. (2.14), setting $q_{\|}=0$ and using the 3D ALDA exchange kernel to obtain $n_{1 \sigma}$.

For $L<L_{2}$, only the lowest subband is occupied (the quantum limit). Equation (2.31) can also be rewritten in terms of the 2D Wigner-Seitz radius $r_{s}^{2 \mathrm{D}}$ as [22]

$$
L_{2}=\sqrt{\frac{3 \pi}{2 N_{s}}}=\pi r_{s}^{2 \mathrm{D}} \sqrt{\frac{3}{2}} \approx 3.85 r_{s}^{2 \mathrm{D}}
$$

Figure 2.2 shows ALDA intersubband excitation spectra at $q_{\|}=0$, in the charge and spin channel, for quantum wells with different numbers of occupied subbands, ranging from $N_{\text {occ }}=1$ to $35 . L$ and $N_{s}$ are chosen such that the average density remains constant at $\bar{n}=0.30 a_{0}^{*-3}$. The insets in the middle show how the density profile becomes more and more square shaped as $N_{\text {occ }}$ increases.

In the quasi-2D limit where $N_{\text {occ }}=1$, the spectra only show a single peak in 
the energy range below 10 a.u.: the intersubband charge plasmon at 5.17 a.u. (left bottom panel) and spin plasmon at 4.53 a.u. (right bottom panel). As more subbands become occupied, the spectra acquire more and more peaks, and eventually approach very simple bulk limits for large $N_{\text {occ }}$.

At $N_{\text {occ }}=35$, the charge-density excitation spectrum is dominated by a single peak at 1.94 a.u., which is the bulk plasmon frequency $\omega_{\text {pl }}$ corresponding to $\bar{n}$. There is also a small peak around 0.22 a.u., which corresponds to the surface plasmon of a large jellium slab with a sharp density profile [41]. On the other hand, the spindensity excitation spectrum has become essentially featureless; in other words, the spin plasmon is seen to disappear in the bulk limit, as expected.

Thus, there is a seamless transition between the 3D bulk plasmon and the intersubband plasmons as the $2 \mathrm{D}$ limit is approached. In this regime, the 3D ALDA (or any $3 \mathrm{D}$ semilocal functional) is appropriate.

\subsubsection{D Limit of intersubband plasmons}

We now focus on the situation where only the first subband is occupied $\left(N_{\text {occ }}=1\right)$, i.e., we consider quantum wells of width $L<L_{2}$. Figure 2.3 shows the intersubband charge and spin plasmon dispersions for quantum wells with $N_{s}=10^{12} \mathrm{~cm}^{-2}$ and $L=100$ and $40 \AA$, respectively, calculated with RPA, ALDA, PBE and PGG. In all cases, the charge plasmon dispersion lies above the spin plasmon dispersion (except for RPA, which has no spin plasmon). However, the position of the intersubband plasmon dispersions relative to the particle-hole ( $\mathrm{p}-\mathrm{h})$ continuum varies.

For the $100 \AA$ wide quantum well we find that the charge plasmon branches are above the p-h continuum and spin plasmon branches are below. For the $40 \AA$ well, 


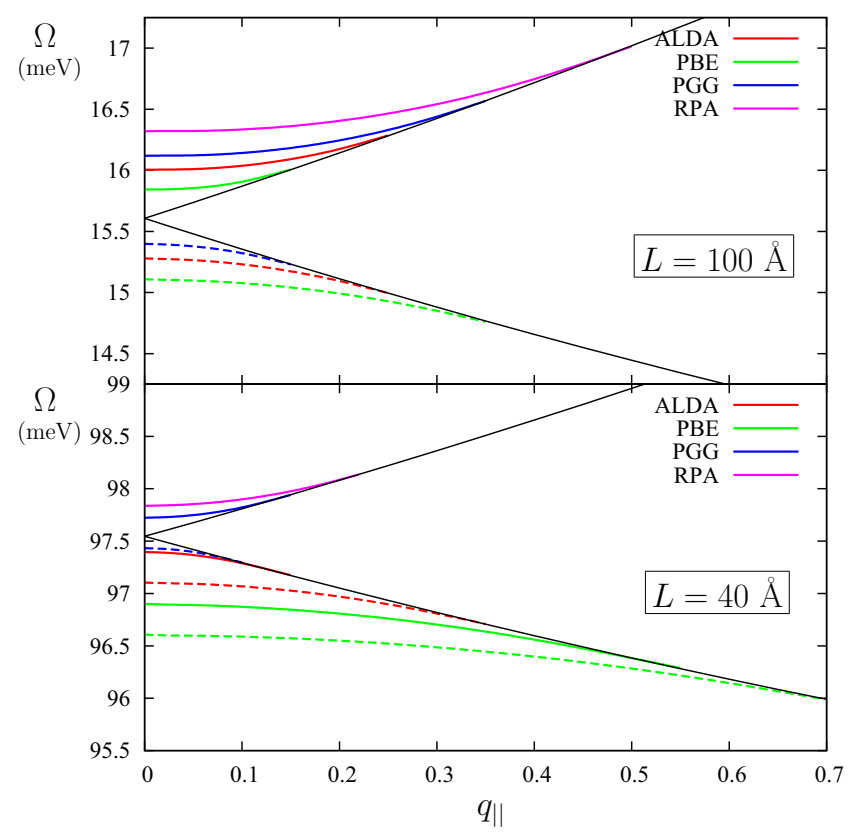

Figure 2.3: Intersubband plasmon dispersions $\Omega\left(q_{\|}\right)$, for $N_{s}=10^{12} \mathrm{~cm}^{-2}$ and well widths $100 \AA$ and $40 \AA$. The black full lines indicate the intersubband p-h continuum. The RPA only gives intersubband charge plasmons; ALDA, PBE and PGG give both charge (full lines) and spin plasmons (dashed lines). ALDA and PBE break down when their charge plasmons falls below the p-h continuum.

however, the charge plasmon branch has moved below [42] the p-h continuum for ALDA and PBE, but not for RPA and PGG. This is a remarkable difference between semilocal and orbital-dependent exchange functionals, and we will now investigate this effect in more detail.

Let us consider the case $q_{\|}=0$ and keep the sheet density $N_{s}$ fixed. As $L \rightarrow 0$, the system transitions from quasi-2D to strictly 2D [33]. In this limit, the intersubband excitation energies become infinitely large, because the system is so strongly confined in the plane that density fluctuations perpendicular to the quantum well plane (see Fig. 2.1) become impossible. However, it is interesting to observe how the 


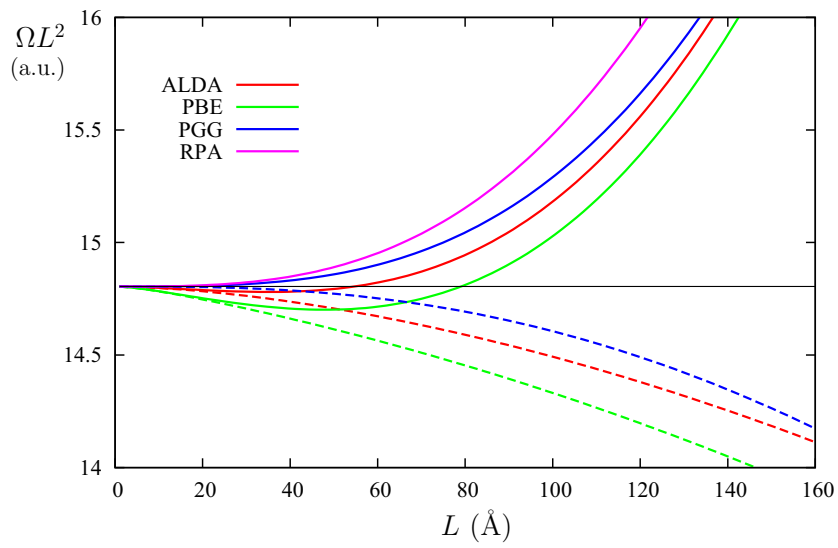

Figure 2.4: Intersubband plasmon energies, at $q_{\|}=0$, versus well width $L$, for $N_{s}=10^{12} \mathrm{~cm}^{-2}$. The horizontal line indicates the lowest p-h transition $\omega_{21}$ (all energies are scaled by $L^{2}$ ). The RPA only gives intersubband charge plasmons; ALDA, PBE and PGG give both charge (full lines) and spin plasmons (dashed lines). ALDA and $\mathrm{PBE}$ break down when the charge plasmon falls below the $\mathrm{p}-\mathrm{h}$ line.

intersubband plasmons behave as this limit is approached. This is shown in Fig. 2.4.

We have calculated the $q_{\|}=0$ intersubband charge and spin plasmon energies with RPA (charge plasmon only), ALDA, PBE, and PGG. According to Eq. (2.6) the lowest $\mathrm{p}$ - $\mathrm{h}$ transition energy is $\omega_{21}=3 \pi^{2} / 2 L^{2}$. Hence, $\omega_{21} L^{2}$ is constant, as indicated by the thin horizontal line in Fig. 2.4. As $L$ becomes smaller, the plasmon energies (scaled by $L^{2}$ ) approach and eventually merge with the p-h line.

The RPA plasmon energy follows from Eq. (2.18) as

$$
\left(\Omega_{c}^{\mathrm{RPA}} L^{2}\right)^{2}=\frac{9 \pi^{4}}{4}+\frac{20 \pi N_{s} L^{3}}{3}
$$

where the Hartree part of the intersubband matrix element (2.19) is given by

$$
-2 \pi \int d z \int d z^{\prime} \varphi_{1}(z) \varphi_{2}(z)\left|z-z^{\prime}\right| \varphi_{1}\left(z^{\prime}\right) \varphi_{2}\left(z^{\prime}\right)=\frac{20 L}{9 \pi}
$$


Hence, the RPA charge plasmons are always shifted above the p-h line, but the separation vanishes as $L \rightarrow 0$.

In ALDA, we find

$$
\begin{aligned}
& \left(\Omega_{c}^{\mathrm{ALDA}} L^{2}\right)^{2}=\frac{9 \pi^{4}}{4}+\frac{20 \pi N_{s} L^{3}}{3}-c_{1}\left(48 \pi^{2} N_{s} L^{5}\right)^{1 / 3} \\
& \left(\Omega_{s}^{\mathrm{ALDA}} L^{2}\right)^{2}=\frac{9 \pi^{4}}{4}-c_{1}\left(48 \pi^{2} N_{s} L^{5}\right)^{1 / 3}
\end{aligned}
$$

where $c_{1}=\int_{0}^{\pi} d x \sin ^{2}(2 x) \sin ^{2 / 3}(x)=1.20027$. For the PBE and PGG plasmon energies no simple analytic expressions exist; however, numerical evaluation is straightforward using the formulas in Appendix B.

As can be seen from Fig. 2.4, the ALDA and PBE charge plasmons cross over the p-h line: this happens at $L=54.6 \AA$ in ALDA and at $L=79 \AA$ in PBE. No such crossover is observed for PGG.

The critical width $L_{\text {crit }}^{\text {inter }}$ at which the crossover occurs in ALDA and PBE is plotted in Fig. 2.5 as a function of the sheet density $N_{s}$. In ALDA we can use Eq. (2.35) to find the analytical result

$$
L_{\text {crit }}^{\text {inter }}=\frac{3 c_{1}^{3 / 4}}{5 \sqrt{N_{s}}}\left(\frac{5}{4 \pi}\right)^{1 / 4}=\frac{0.546}{\sqrt{N_{s}}} \text { a.u. }
$$

For PBE, we obtain numerically $L_{\text {crit }}^{\text {inter }}=0.79 / \sqrt{N_{s}}$ a.u. In terms of the $2 \mathrm{D}$ WignerSeitz radius, this becomes $L_{\text {crit }}^{\text {inter }}=0.975 r_{s}^{2 \mathrm{D}}$ and $1.40 r_{s}^{2 \mathrm{D}}$ for ALDA and PBE, respectively. In the case of ALDA, this is about 4 times smaller than $L_{2}$ [Eq. (2.32)], the width of the quantum well below which only the lowest subband is occupied; in the case of PBE, it is about 3 times smaller.

In PGG, we find that the charge and spin plasmons always lie above and below 


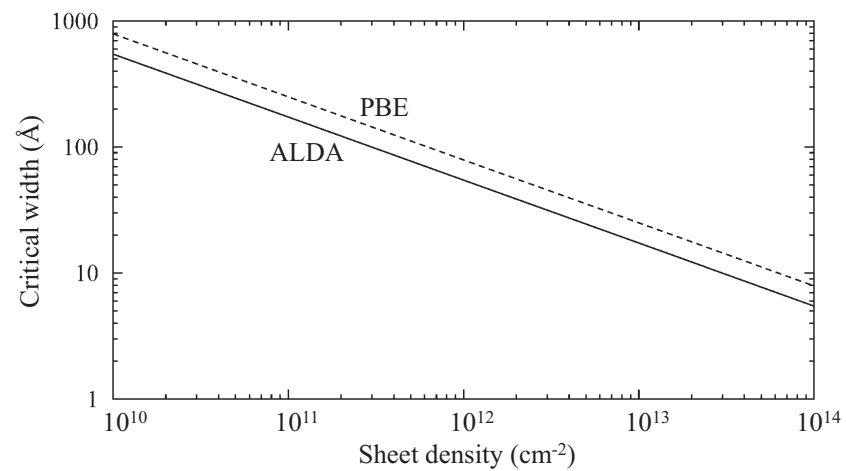

Figure 2.5: Critical width $L_{\text {crit }}^{\text {inter }}$ at which the intersubband plasmon breakdown occurs, as a function of sheet density $N_{s}$. Full line: ALDA, dashed line: PBE.

the $\mathrm{p}$-h continuum, respectively. This is similar to the case of excitation energies in atoms, where the bare Kohn-Sham exictations are found to lie between the singlet and triplet excitations $[43,44,45]$. Hence, the crossover of ALDA and PBE indicates a general failure of semilocal functionals in the 2D limit of intersubband transitions.

However, it is important to note that this failure does not appear to be a catastrophic breakdown, as in the case of the diverging exchange energy that we discussed in the Introduction. The intersubband plasmons may have a wrong position with respect to the $\mathrm{p}-\mathrm{h}$ continuum, but they still exist as collective modes, and deviate not too far from the PGG results. Furthermore, the separation between charge and spin plasmons (the analog of the singlet-triplet splitting in atoms) remains well described in ALDA and PBE for all $L$.

In practice, the width of quantum wells is limited by the underlying material (for GaAs, the lattice constant is $5.65 \AA$ ). Typical semiconductor quantum wells have widths of several hundreds of $\AA$, so that one is usually sufficiently far away from the critical widths where the ALDA breaks down for the intersubband dynamics, except for situations where $N_{s}$ is very small. 


\subsubsection{D limit of intrasubband plasmons}

Let us now consider the intrasubband plasmons in a quantum well with $N_{\text {occ }}=1$, in the limit where $L \rightarrow 0$. For convenience, we shift the bottom of the quantum well potential such that the lowest subband level $\varepsilon_{1}=0$. Assuming, furthermore, that the second and higher subband levels are energetically well separated from the lowest subband, the response function (2.11) is given by

$$
\chi_{s, \sigma \sigma^{\prime}}\left(\mathbf{k}_{\|}, z, z^{\prime}, \omega\right)=\delta_{\sigma \sigma^{\prime}} \Phi\left(z, z^{\prime}\right) \chi_{0}^{2 \mathrm{D}}\left(k_{\|}, \omega\right),
$$

where $\chi_{0}^{2 \mathrm{D}}\left(k_{\|}, \omega\right)$ is the 2D Lindhard function, and where we abbreviate $\Phi\left(z, z^{\prime}\right)=$ $\varphi_{1}^{2}(z) \varphi_{1}^{2}\left(z^{\prime}\right)$. The response equation (2.10) for the eigenmodes then becomes

$$
n_{1}\left(q_{\|}, z^{\prime}, \Omega\right)=\int d z_{1} \Phi\left(z^{\prime}, z_{1}\right) \chi_{0}^{2 \mathrm{D}}\left(q_{\|}, \Omega\right) \int d z_{2} f_{\mathrm{Hxc}}\left(q_{\|}, z_{1}, z_{2}\right) n_{1}\left(q_{\|}, z_{2}, \Omega\right) .
$$

Multiply both sides with $\varphi_{1}^{2}(z) f_{\mathrm{Hxc}}\left(q_{\|}, z, z^{\prime}\right)$ and integrate over $z$ and $z^{\prime}$. Then, $n_{1}$ cancels out and we are left with the condition

$$
1=\int d z \int d z^{\prime} \Phi\left(z, z^{\prime}\right)\left[\frac{2 \pi}{q_{\|}} e^{-q_{\|}\left|z-z^{\prime}\right|}+f_{\mathrm{xc}}\left(q_{\|}, z, z^{\prime}\right)\right] \chi_{0}^{2 \mathrm{D}}\left(q_{\|}, \Omega\right) .
$$

The intrasubband plasmons of the quasi-2D quantum well are those frequencies $\Omega$ where Eq. (2.40) is satisfied. The question is now this: if $L \rightarrow 0$, will Eq. (2.40) turn into Eq. (2.26) for the 2D plasmons?

A straightforward calculation shows that this is indeed the case for the Hartree 
part, as expected. Using the particle-in-a-box wave function (2.5) we obtain

$$
\begin{aligned}
& \int d z \int d z^{\prime} \Phi\left(z, z^{\prime}\right) e^{-q_{\|}\left|z-z^{\prime}\right|}=\frac{q_{\|} L}{\left(q_{\|}^{2} L^{2}+4 \pi^{2}\right)^{2}} \\
& \quad \times\left\{3 q_{\|}^{2} L^{2}+20 \pi^{2}+\frac{32 \pi^{4}}{q_{\|}^{3} L^{3}}\left(e^{-q_{\|} L}-1+q_{||} L\right)\right\} \\
& \quad \longrightarrow 1 \text { for } L \rightarrow 0 .
\end{aligned}
$$

For the PGG exchange kernel, it is straightforward to show that

$$
\int d z \int d z^{\prime} \Phi\left(z, z^{\prime}\right) f_{\mathrm{x}}^{\mathrm{PGG}}\left(q_{\|}, z, z^{\prime}\right) \longrightarrow f_{\mathrm{x}, 2 \mathrm{D}}^{\mathrm{PGG}}\left(q_{\|}\right)
$$

for $L \rightarrow 0$, where $f_{\mathrm{x}}^{\mathrm{PGG}}\left(q_{\|}, z, z^{\prime}\right)$ and $f_{\mathrm{x}, 2 \mathrm{D}}^{\mathrm{PGG}}\left(q_{\|}\right)$are given in Appendix $\mathrm{B}$, see Eqs. (B.4) and (B.6). Thus, the PGG exchange kernel behaves correctly in the 2D limit.

However, it is hardly surprising to find that the ALDA does not give the correct 2D limit. We have

$$
\int d z \int d z^{\prime} \Phi\left(z, z^{\prime}\right) f_{\mathrm{x}, 3 \mathrm{D}}^{\mathrm{ALA}}\left(z, z^{\prime}\right)=-\frac{2 c_{2}}{3 \pi}\left(\frac{6}{\pi L}\right)^{1 / 3} n_{2 \mathrm{D}}^{-2 / 3}
$$

where $c_{2}=\int_{0}^{\pi} d x \sin ^{8 / 3}(x)=1.4003$. This clearly disagrees with the form of $f_{\mathrm{x}, 2 \mathrm{D}}^{\mathrm{ALDA}}=$ $-\sqrt{2 / \pi n_{2 \mathrm{D}}}$, and in fact diverges as $L \rightarrow 0$. Other semilocal functionals such as PBE show similar trends.

Figure 2.6 shows the plasmon dispersions in the strictly 2D limit, calculated by solving Eq. (2.26). The ALDA and PGG calculations were done with the 2D exchange kernels $f_{\mathrm{x}, 2 \mathrm{D}}^{\mathrm{ALDA}}$ and $f_{\mathrm{x}, 2 \mathrm{D}}^{\mathrm{PGG}}$, respectively. The upper boundary of the particle-hole continuum is given by the relation $\tilde{\Omega}={\tilde{q_{\|}}}^{2} / 2+\tilde{q_{\|}}$, where $\tilde{q_{\|}}=q_{\|} / k_{F}^{2 \mathrm{D}}$ and $\tilde{\Omega}=\Omega /\left(k_{F}^{2 \mathrm{D}}\right)^{2}$. One observes that the RPA plasmon dispersion always lies above ALDA and PGG, 

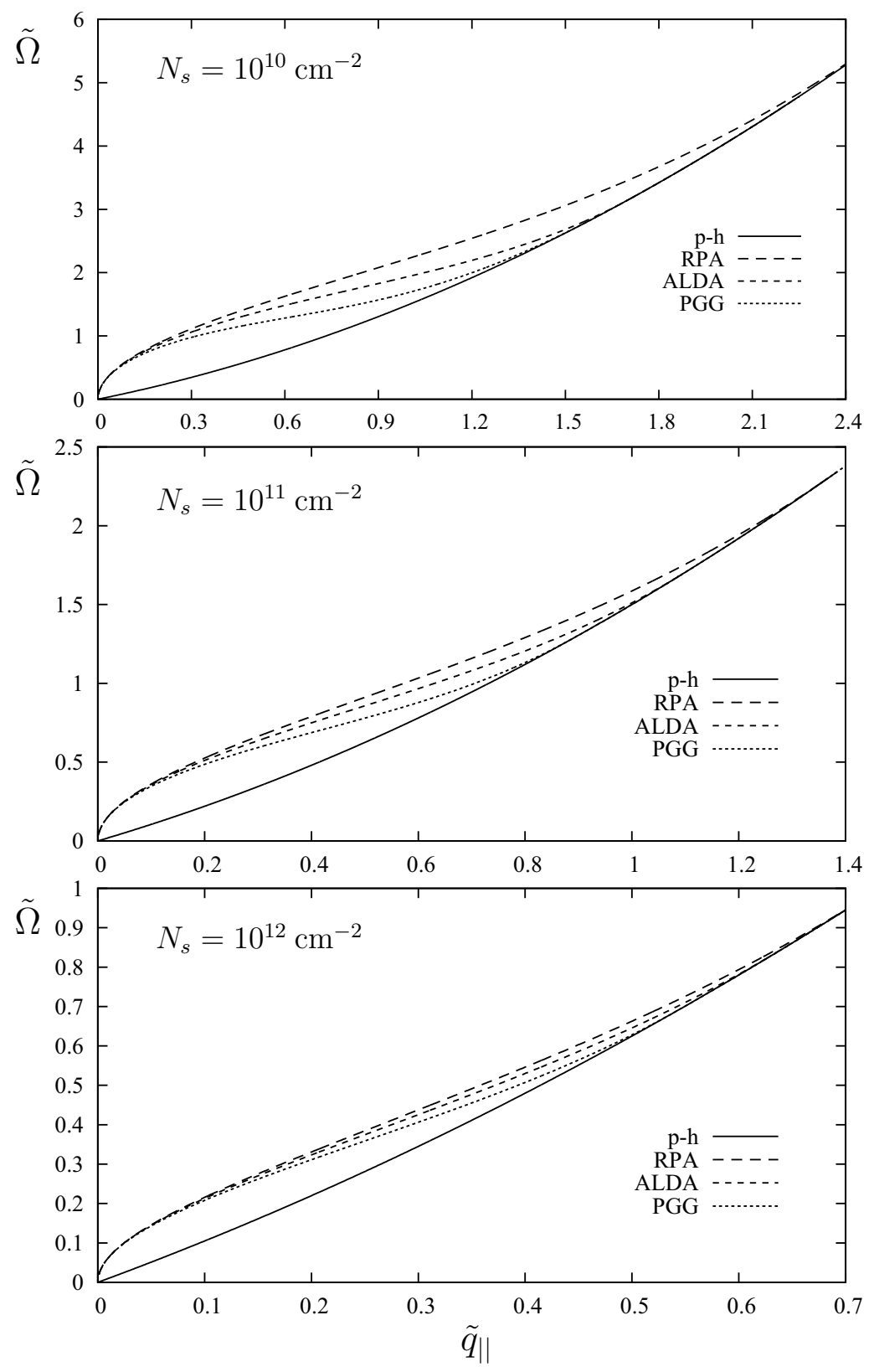

Figure 2.6: Plasmon dispersions $\Omega\left(q_{\|}\right)$for strictly 2D systems with sheet densities $N_{s}=10^{10}, 10^{11}$, and $10^{12} \mathrm{~cm}^{-2}$, calculated with RPA, 2D ALDA and PGG. The full lines denote the upper boundaries of the particle-hole $(\mathrm{p}-\mathrm{h})$ continuum. Here, $\tilde{q_{\|}}=q_{\|} / k_{F}^{2 \mathrm{D}}$ and $\tilde{\Omega}=\Omega /\left(k_{F}^{2 \mathrm{D}}\right)^{2}$. 
reflecting the downshift of excitation energies caused by exchange.

Figure 2.7 compares the intrasubband plasmon dispersions of PGG and 3D ALDA for well widths $L=\lambda L_{2}$, where we let the scaling parameter $\lambda$ take on values between 1 and 0.001 [recall that $L_{2}$, Eq. (2.31), is the maximum well width for which only the lowest subband is occupied for a given $N_{s}$. The sheet density is $N_{s}=10^{10} \mathrm{~cm}^{-2}$, and we have $L_{2}=217 \mathrm{~nm}$.

As expected, PGG nicely approaches the 2D limit that was shown in Fig. 2.6. For $\lambda<0.01$, the intrasubband plasmon dispersion becomes indistinguishable from the strictly $2 \mathrm{D}$ limit.

The situation is drastically different for the ALDA. As $\lambda$ decreases from 1 to 0.1 , the intrasubband dispersion appears to approach the 2D limit. However, below $\lambda=0.1$ the 3D ALDA starts to fail. The performance at small values of $q_{\|}$is still good, but the plasmon branch enters the particle-hole continuum too soon, and the trend of the entry point as a function of $q_{\|}$is reversed. Eventually, as $\lambda \rightarrow 0$, the intrasubband plasmon completely disappears, rather than approaching the 2D plasmon shown in Fig. 2.6.

We have repeated these calculations for several different values of the sheet density $N_{s}$, focusing on the wavevector $\tilde{q}_{\| p-h}$ where the intrasubband plasmon enters the p-h continuum, as indicated by the blue squares in Fig. 2.7.

Figure 2.8 shows $\tilde{q}_{\| p-h}$ versus the well width scaling factor $\lambda$ for $N_{s}=10^{10}, 10^{11}$, $10^{12}$, and $10^{13} \mathrm{~cm}^{-2}$, calculated with ALDA and PGG. For PGG we see in each case that $\tilde{q}_{|| p-h}$ smoothly approaches its limiting value for the strictly 2D plasmon, shown by the dashed line. The ALDA initially approaches the $2 \mathrm{D}$ limit as $\lambda$ decreases from 1. However, around $\lambda=0.1$ all ALDA curves turn around and rapidly drop off, 


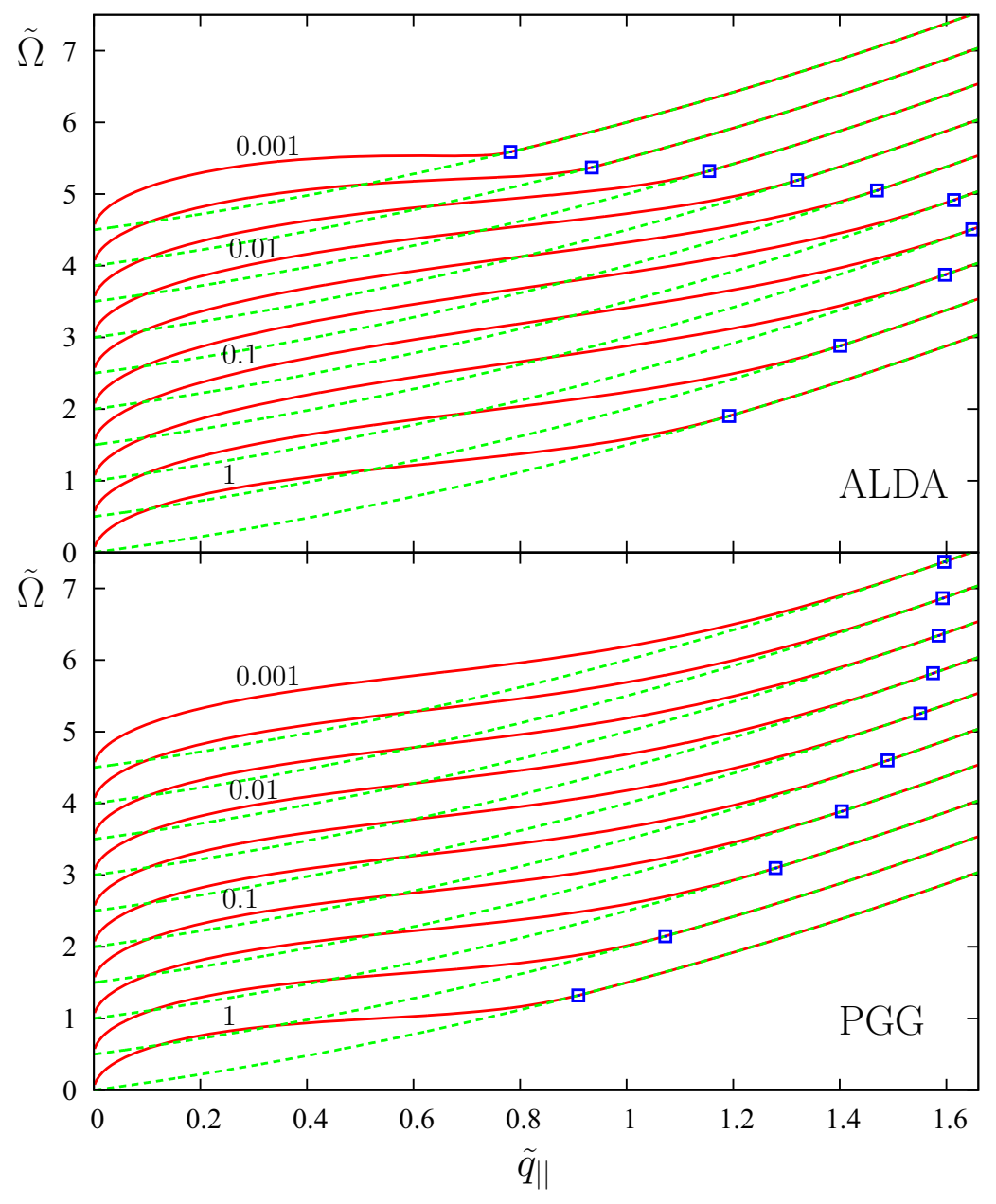

Figure 2.7: Intrasubband plasmon dispersions for quantum wells with sheet density $N_{s}=10^{10} \mathrm{~cm}^{-2}$, for different widths $L=\lambda L_{2}$, where $\lambda$ takes on the values $1,0.5,0.2$, $0.1,0.05,0.02,0.01,0.005,0.002$, and 0.001. $L_{2}=217 \mathrm{~nm}$ is the largest width for which only the lowest subband is occupied. The individual plasmon dispersions are offset for clarity. The dashed lines are the upper boundaries of the $\mathrm{p}$-h continuum. The squares indicate the wavevector $\tilde{q}_{\mid p-h}$ where the plasmons enter the p-h continuum. Top panel: 3D ALDA. Bottom panel: PGG. 


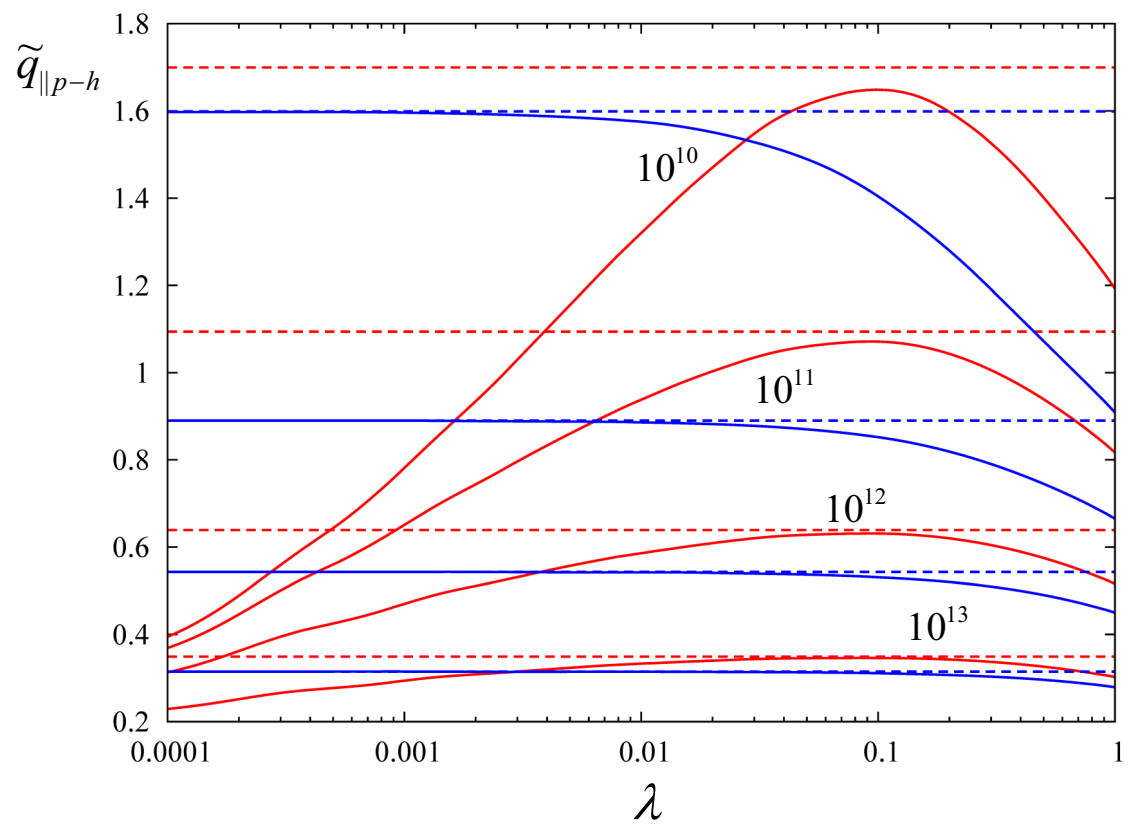

Figure 2.8: Wavevector $\tilde{q}_{\| p-h}$ at which the intrasubband plasmon merges with the p-h continuum, plotted versus well width scaling factor $\lambda$, calculated with PGG (blue) and ALDA (red). The dashed lines indicate the respective limits for the strictly 2D case. The calculations were done for sheet densities $N_{s}=10^{10}, 10^{11}, 10^{12}$, and $10^{13} \mathrm{~cm}^{-2}$, as indicated. The breakdown of the 3D ALDA occurs around $\lambda=0.1$ for all $N_{s}$.

moving away from the 2D limit.

Thus, we find that the 3D ALDA exchange kernel behaves reasonably as long as the well width is sufficiently large. The breakdown for intrasubband (in-plane) dynamics occurs for $L_{\text {crit }}^{\text {intra }} \approx 0.1 L_{2} \approx 0.4 r_{s}^{2 \mathrm{D}}$. Interestingly, this is significantly smaller than the critical intersubband width $L_{\text {crit }}^{\text {inter }} \approx r_{s}^{2 \mathrm{D}}$, see Section 2.2.2. 


\subsection{Conclusions}

In this chapter we have carried out systematic numerical studies of the electron dynamics in quantum wells whose width $L$ crosses over from the $3 \mathrm{D}$ to the quasi-2D regime (where only the lowest subband is occupied, but the finite size is still relevant) and finally to the strictly $2 \mathrm{D}$ limit (where $L=0$ ). The purpose was a comparison of different classes of exchange kernels in TDDFT: standard semilocal kernels (such as ALDA and PBE) and nonlocal kernels (such as PGG and ISTLS). ALDA and PBE are based on the electron gas as reference system, whereas PGG and ISTLS are orbital functionals, whose definition does not invoke any reference system.

The main conclusion does not come as a surprise: ALDA and PBE fail in the 3D-2D crossover, PGG succeeds. This is already well known for the ground state $[22,23,24,25]$, and there was no reason to expect otherwise for the dynamical case. However, the details are interesting and of practical relevance.

First of all, we discover a universal behavior of the breakdown of the inter- and intrasubband dynamics in 3D ALDA. At a critical well width of $L_{\text {crit }}^{\text {inter }} \approx r_{s}^{2 \mathrm{D}}$, intersubband plasmons are no longer qualitatively correctly described (the charge plasmon falls below the single-particle excitation $\left.\omega_{21}\right)$. For well widths below $L_{\text {crit }}^{\text {intra }} \approx 0.4 r_{s}^{2 \mathrm{D}}$, intrasubband plasmon dispersions start to become suppressed compared to the $2 \mathrm{D}$ limit. The interesting finding is thus that $L_{\text {crit }}^{\text {intra }}<L_{\text {crit }}^{\text {inter }}$, so the in-plane dynamics appears to be well described using the 3D ALDA down to much smaller widths than the out-of-plane dynamics.

Compared to the ground state, the failure of the (semi)local xc functionals in the dynamical case is of a different nature. In fact, while the exchange energy diverges for $L \rightarrow 0$, intersubband plasmons can still be reasonably described (apart from 
the fact that they drop below the p-h continuum [42], which is an artifact of these functionals). In turn, intrasubband plasmon dispersions become suppressed and cease to exist, instead of approaching the limit of 2D plasmons.

In practice, it is important to know for what quantum well widths the 3D ALDA is still applicable. For instance, if $N_{s}=10^{11} \mathrm{~cm}^{-2}$ (which is a very typical value for many semiconductor quantum well samples), we find $L_{\text {crit }}^{\text {inter }}=17 \mathrm{~nm}$ for GaAs, which is rather narrow. Higher sheet densities allow one to push this limit to even narrower wells; and the breakdown for intrasubband dynamics occurs at even smaller well widths, as low as a few $\AA$. This is certainly good news, considering the popularity of the ALDA and its ease of implementation. We also find that these values can be significantly higher for the PBE; in other words, using gradient-corrected xc functionals for quantum wells does not seem to pay off [46].

Clearly, the best option to describe the dynamics in strongly confined systems is using nonlocal orbital functionals such as PGG or ISTLS, since these are not tied to a particular choice of reference system (such as the $2 \mathrm{D}$ or $3 \mathrm{D}$ ALDA) and hence have no problem with dimensional crossover.

Finally, let us say a few words about correlation. In the ground state [22, 23, $24,25]$, it was observed that local and semilocal correlation functionals break down in a similar manner as exchange functionals. This will also be the case for the dynamics. However, nonlocal, orbital-dependent correlation functionals are much more complicated than exchange functionals; for instance, implementing the ISTLS beyond exchange in linear response will remain a task for the future.

There is another aspect of correlation that is unique to the dynamical case, namely, it leads to dissipation of plasmon excitations even outside the particle-hole continuum. 
Plasmon damping in quantum wells has been studied within time-dependent currentDFT $[47,48,49,50]$, using the complex and frequency-dependent xc kernel of Vignale and Kohn $[51,52,53]$. This xc kernel is a local approximation of the current, and can lead to overdamping of charge plasmons if the system becomes too inhomogeneous $[48,54]$. The effect is even more dramatic for spin plasmons, where the damping due to the spin Coulomb drag effect is significantly overestimated using a local approximation [55]. Again, it is found that the cure to this overdamping is provided by orbital functionals [56]. 


\section{Chapter 3}

\section{Spin precession and spin waves in a chiral electron gas: beyond Larmor's theorem}

Larmor's theorem $[57,58]$ states that in a system of charges, all with the same chargemass ratio $q / m$, moving in a centrally symmetric electrostatic potential and in a sufficiently weak magnetic field $\mathbf{B}$, the charges precess about the direction of the magnetic field with the frequency

$$
\Omega_{L}=g \frac{q B}{2 m}
$$

(in SI units), where $g$ is the gyromagnetic ratio or $g$-factor.

In condensed-matter physics, Larmor's theorem applies to the long-wavelength limit of spin-wave excitations in magnetic systems which are invariant under spin rotation [59]. In particular, the electrons in a two-dimensional electron gas (2DEG) in the presence of a constant uniform magnetic field carry out a precessional motion 
at the single-particle Larmor frequency, despite the presence of Coulomb interactions.

If spin-rotational invariance is broken - for instance, in the presence of spin-orbit coupling (SOC) - Larmor's theorem is no longer guaranteed to hold, and there will be corrections to $\Omega_{L}$. This was experimentally observed over three decades ago for a 2DEG in a GaAs/AlGaAs heterostructure, using electron spin resonance (ESR) [60]. Subsequently, several theoretical studies addressed Larmor's theorem in collective spin excitations in 2DEGs $[61,62,63,64,65,66,67]$. The corrections to $\Omega_{L}$ are caused by a subtle interplay between SOC and Coulomb many-body effects, which poses significant formal and computational challenges; on the other hand, this offers interesting opportunities for the experimental determination of SOC parameters and the study of many-body interactions.

In this chapter, we present a joint experimental and theoretical study of the spinwave dispersions of a partially spin-polarized 2DEG in a semiconductor quantum well. The influence of Rashba and Dresselhaus SOC on collective electronic modes in quantum wells was first theoretically predicted to cause an angular modulation of the intersubband plasmon dispersion $[68,69]$. The effect was later experimentally confirmed [70], and then extended to spin-wave dispersions [71, 72, 73, 74].

In the absence of SOC, the real part of the spin-wave dispersion of a paramagnetic 2DEG has the following form for small wave vectors [75]:

$$
\hbar \omega_{\mathrm{sw}}(\mathbf{q})=Z+\frac{1}{2} S_{\mathrm{sw}} q^{2}
$$

where $Z$ is the bare Zeeman energy, and $S_{\text {sw }}$ is the spin-wave stiffness, which depends on Coulomb many-body effects (explicit expressions for $Z$ and $S_{\mathrm{sw}}$ will be given in Section 3.1). Note that for a partially spin polarized 2DEG the spin-wave stiffness 
$S_{\mathrm{sw}}$ is negative; by contrast, for ferromagnetic systems one finds $S_{\mathrm{sw}}>0$ [59].

It was recently discovered in our group [74] that, to first order in the Rashba and Dresselhaus spin-orbit coupling strengths $\alpha$ and $\beta$, the spin-wave dispersion is unchanged apart from a chiral shift by a constant wave vector $\mathbf{q}_{0}$ (defined in Sec. 3.2) which depends on $\alpha, \beta$ and the angle $\varphi$ between the magnetization direction and the [010] crystalline axis (see Fig. 3.1). In other words, to quadratic order in the wave vector, we find

$$
\hbar \omega_{\mathrm{sw}}^{\mathrm{SO}}(\mathbf{q})=Z+\frac{1}{2} S_{\mathrm{sw}}\left|\mathbf{q}+\mathbf{q}_{0}\right|^{2}+\mathcal{O}\left(\alpha^{2}, \beta^{2}\right)
$$

The spin-wave stiffness $S_{\mathrm{sw}}$ remains unchanged, to leading order in $\alpha, \beta$. The physical interpretation is that the spin wave behaves as if it were transformed into a spin-orbit twisted reference frame. This opens up new possibilities for manipulating spin waves, which may lead to new applications in spintronics.

To account for higher-order SOC effects in the spin-wave dispersion, it is sensible to rewrite Eq. (3.3) in a more general manner:

$$
\omega_{\mathrm{sw}}^{\mathrm{SO}}(\mathbf{q})=E_{0}(\varphi)+E_{1}(\varphi) q+E_{2}(\varphi) q^{2}
$$

where the coefficients $E_{0}, E_{1}$ and $E_{2}$ depend on the propagation direction $\varphi$ (see Fig. 3.1). From Eq. (3.3), the linear coefficient is given to leading order in SOC by $E_{1}(\varphi)=S_{\mathrm{sw}} \mathbf{q} \cdot \mathbf{q}_{0} / q$, which can be expressed as $[74]$

$$
E_{1}(\varphi)=-\frac{2}{\zeta} \frac{Z}{\left(Z^{*}-Z\right)}(\alpha+\beta \sin 2 \varphi)
$$

where $\zeta$ is the spin polarization of the $2 \mathrm{DEG}$, and $Z^{*}$ is the renormalized Zeeman splitting, to be defined below in Section 3.1.2. 
We will present a linear-response approach based on time-dependent densityfunctional theory (TDDFT) which allows us to obtain analytical results for $E_{0}$, to second order in $\alpha, \beta$, and numerical results for $E_{1}$ and $E_{2}$ to all orders in SOC. The breaking of Larmor's theorem is expressed in the coefficient $E_{0}$, which has $\varphi$ dependent corrections to $Z$. In Section 3.3 we will obtain the following result to leading order in SOC:

$$
E_{0}(\varphi)=Z+\frac{2 \pi N_{s}}{Z^{*} f_{T}}\left[\left(\alpha^{2}+\beta^{2}\right)\left(3 f_{T}+2\right)+2 \alpha \beta \sin (2 \varphi)\left(f_{T}+2\right)\right]
$$

where $f_{T}=\left(Z-Z^{*}\right) / Z^{*}$.

Our analytical and numerical results will be compared with experimental results, obtained via inelastic light scattering in a CdMnTe quantum well sample. The experiment was carried out by our collaborators, Florent Perez and Florent Baboux, at the Universit Pierre et Marie Curie in Paris [76]. By fitting $E_{0}, E_{1}$ and $E_{2}$ we are able to extract values for $Z^{*}, \alpha$ and $\beta$ and present evidence for the $\varphi$ dependence of $E_{0}$ and $E_{2}$, which had not been considered in the earlier work done by our group [74]. Comparison to theory shows significant deviations from the standard approximation in TDDFT, the adiabatic local-density approximation (ALDA). This provides new incentives to search for better exchange-correlation functionals for transverse spin excitations of electronic systems.

This chapter is organized as follows. In Section 3.1 we discuss Larmor's theorem without SOC: first, for completeness, we present a general proof for interacting manybody systems, and then we discuss Larmor's theorem from a TDDFT perspective. This will lead to a new constraint for the exchange-correlation kernel of linear-response TDDFT. In Section 3.2 we consider the electronic states in a quantum well with SOC 
and an in-plane magnetic field. Section 3.3 contains the derivation of the spin-wave dispersions from linear-response TDDFT, in the presence of SOC. In Section 3.4 we compare our theory with experimental results and discuss our findings. Section 3.5 gives our conclusions.

\subsection{Larmor's theorem}

In this section we consider Larmor's theorem in a 2DEG, from a general many-body perspective (the proof given in Sec. 3.1.1 is not new [58] but included here to keep the chapter self-contained), and from the perspective of TDDFT. This will set the stage for the discussions in the following sections where the effects of SOC are included.

\subsubsection{Long-wavelength limit of spin waves a 2DEG}

Let us consider a 2DEG in the presence of a uniform magnetic field $\mathbf{B}=B \hat{e}_{z}$, where $\hat{e}_{z}$ is a unit vector lying in the plane of the 2DEG. The Hamiltonian is

$$
\hat{H}=\sum_{i}\left[\frac{\hat{\mathbf{p}}_{i}^{2}}{2 m}+\frac{Z}{2} \hat{\sigma}_{z, i}\right]+\frac{e^{2}}{2} \sum_{i j} \frac{1}{\left|\mathbf{r}_{i}-\mathbf{r}_{j}\right|}
$$

Here, $m$ and $e$ are the electron mass and charge, $Z=g \mu_{B} B$ is the Zeeman energy (the splitting between the spin-up and spin-down bands), and $\mu_{B}=|e| \hbar / 2 m$ is the Bohr magneton. For a 2DEG embedded in a semiconductor, $m, e$, and $g$ are replaced by the effective mass, charge and g-factor, $m^{*}, e^{*}$ and $g^{*}$, where $g^{*}$ could be a positive or negative number.

Since the magnetic field is applied in the plane of the 2DEG (in this section, we 
assume for simplicity that the 2DEG has zero thickness), its only effect is on the electron spin and there is no Landau level quantization. Later on, when we discuss quantum wells of finite width, we will exclude situations where the magnetic length $l_{B}=\sqrt{\hbar /|e B|}$ is smaller than the well width, and hence continue to disregard any orbital angular momentum contributions.

Let us define the spin-wave operator $[58,77,78,79]$

$$
\hat{S}_{+, \mathbf{q}}=\frac{1}{2} \sum_{i} \hat{\sigma}_{+, i} e^{-i \mathbf{q} \cdot \mathbf{r}_{i}}
$$

where $\hat{\sigma}_{+}=\hat{\sigma}_{x}+i \hat{\sigma}_{y}$. This operator satisfies the Heisenberg equation of motion

$$
\frac{d}{d t} \hat{S}_{+, \mathbf{q}}=\frac{1}{i \hbar}\left[\hat{S}_{+, \mathbf{q}}, \hat{H}\right]=i \omega_{\mathrm{sw}}(\mathbf{q}) \hat{S}_{+, \mathbf{q}},
$$

where $\omega_{\text {sw }}(\mathbf{q})$ is the spin-wave frequency dispersion of the 2 DEG. We are interested in the special case $\mathbf{q}=0$, and abbreviate $\omega_{\mathrm{sw}}(\mathbf{q}=0)=\omega_{\mathrm{sw}, 0}$. The operator $\hat{S}_{+, 0}=$ $\frac{1}{2} \sum_{i} \hat{\sigma}_{+, i}$ commutes with the kinetic and electron-electron interaction parts of $\hat{H}$, and we obtain

$$
\left[\hat{S}_{+, 0}, \hat{H}\right]=\frac{Z}{4} \sum_{i}\left[\hat{\sigma}_{+, i}, \hat{\sigma}_{z, i}\right]=-Z \hat{S}_{+, 0}
$$

where we used the standard commutation relations between the Pauli matrices $\hat{\sigma}_{x}$, $\hat{\sigma}_{y}$ and $\hat{\sigma}_{z}$. Together with Eq. (3.9), this yields

$$
\frac{d}{d t} \hat{S}_{+, 0}=\frac{i}{\hbar} Z \hat{S}_{+, 0}
$$

and hence

$$
\hbar \omega_{\mathrm{sw}, 0}=Z
$$


Larmor's theorem thus says that the long-wavelength limit of the spin-wave dispersion of a 2DEG is given by the bare Zeeman energy, regardless of the presence of Coulomb interactions. By comparison with Eq. (3.1) we have $\Omega_{L}=Z / \hbar$.

\subsubsection{TDDFT perspective}

TDDFT is a formally exact approach to calculate excitations in electronic systems $[29,34]$. In the most general case of a magnetic system, TDDFT can be formulated using the spin-density matrix $\underline{n}$ as basic variable, whose elements are defined as

$$
n_{\sigma \sigma^{\prime}}(\mathbf{r}, t)=\left\langle\Psi(t)\left|\hat{\psi}_{\sigma^{\prime}}^{\dagger}(\mathbf{r}) \hat{\psi}_{\sigma}(\mathbf{r})\right| \Psi(t)\right\rangle
$$

where $\Psi(t)$ is the time-dependent many-body wave function, and $\hat{\psi}_{\sigma}(\mathbf{r}), \psi_{\sigma^{\prime}}^{\dagger}(\mathbf{r})$ are fermionic field operators for spins $\sigma$ and $\sigma^{\prime}$, respectively. The spin-density matrix is diagonal for spatially uniform magnetic fields if the spin quantization axis is along the direction of the field. However, spin-flip excitations involve the transverse (offdiagonal) spin-density matrix response.

The frequency- and momentum-dependent linear-response equation for a 2DEG has the following form:

$$
n_{\sigma \sigma^{\prime}}^{(1)}(\mathbf{q}, \omega)=\sum_{\tau \tau^{\prime}} \chi_{\sigma \sigma^{\prime}, \tau \tau^{\prime}}^{\mathrm{int}}(\mathbf{q}, \omega) v_{\tau \tau^{\prime}}^{(1)}(\mathbf{q}, \omega)
$$

where $v_{\tau^{\prime} \tau^{\prime}}^{(1)}(\mathbf{q}, \omega)$ is a spin-dependent perturbation, and $\chi_{\sigma \sigma^{\prime}, \tau \tau^{\prime}}^{\text {int }}(\mathbf{q}, \omega)$ is the spindensity matrix response function of the interacting many-body system. 
The TDDFT counterpart of Eq. (3.13) is

$$
n_{\sigma \sigma^{\prime}}^{(1)}(\mathbf{q}, \omega)=\sum_{\tau \tau^{\prime}} \chi_{\sigma \sigma^{\prime}, \tau \tau^{\prime}}(\mathbf{q}, \omega) v_{\tau \tau^{\prime}}^{(1) \mathrm{eff}}(\mathbf{q}, \omega)
$$

where $\chi_{\sigma \sigma^{\prime}, \tau \tau^{\prime}}(\mathbf{q}, \omega)$ is the response function of the corresponding noninteracting 2DEG, and the effective perturbation is

$$
\delta v_{\tau \tau^{\prime}}^{(1) \mathrm{eff}}(\mathbf{q}, \omega)=v_{\tau \tau^{\prime}}^{(1)}(\mathbf{q}, \omega)+\sum_{\lambda \lambda^{\prime}}\left[\frac{2 \pi}{q}+f_{\tau \tau^{\prime}, \lambda \lambda^{\prime}}^{\mathrm{xc}}(\mathbf{q}, \omega)\right] n_{\lambda \lambda^{\prime}}^{(1)}(\mathbf{q}, \omega)
$$

Here, $f_{\tau \tau^{\prime}, \lambda \lambda^{\prime}}^{\mathrm{xc}}(\mathbf{q}, \omega)$ is the exchange-correlation $(\mathrm{xc})$ kernel for the spin-density matrix response of the $2 \mathrm{DEG}$.

Let us now consider a noninteracting spin-polarized 2DEG with the Kohn-Sham Hamiltonian

$$
\hat{h}=\sum_{i}\left[\frac{\hat{\mathbf{p}}_{i}^{2}}{2 m}+\frac{Z^{*}}{2} \hat{\sigma}_{z, i}\right]
$$

which produces two parabolic, spin-split energy bands $\hbar^{2} k^{2} / 2 m+\varepsilon_{\uparrow, \downarrow}$ (spin-up and spin-down are taken with respect to the $z$ axis). In the following let us assume that $\varepsilon_{\uparrow}-\varepsilon_{\downarrow}>0$, so $\zeta<0$. The renormalized Zeeman energy is therefore given by

$$
Z^{*}=\varepsilon_{\uparrow}-\varepsilon_{\downarrow}=Z+v_{\mathrm{xc} \uparrow}-v_{\mathrm{xc} \downarrow}
$$

From the xc energy per particle of a spin-polarized 2DEG [80], $e_{\mathrm{xc}}(n, \zeta)$ (where $n$ and $\zeta$ are the density and spin polarization, respectively), the spin-dependent xc potentials 
are obtained as

$$
\begin{aligned}
& v_{\mathrm{xc} \uparrow}=e_{\mathrm{xc}}+n \frac{\partial e_{\mathrm{xc}}}{\partial n}+(1-\zeta) \frac{\partial e_{\mathrm{xc}}}{\partial \zeta} \\
& v_{\mathrm{xc} \downarrow}=e_{\mathrm{xc}}+n \frac{\partial e_{\mathrm{xc}}}{\partial n}-(1+\zeta) \frac{\partial e_{\mathrm{xc}}}{\partial \zeta}
\end{aligned}
$$

so the renormalized Zeeman energy is [78, 81]

$$
Z^{*}=Z+2 \frac{\partial e_{\mathrm{xc}}}{\partial \zeta}
$$

Now let us calculate the collective spin-flip excitations using linear response theory. Since the ground state of the 2DEG has no transverse spin polarization, the spindensity-matrix response decouples into longitudinal and transverse channels, and we can write the associated noninteracting response functions as

$$
\begin{aligned}
& \underline{\chi}_{L}(\mathbf{q}, \omega)=\left(\begin{array}{ll}
\chi_{\uparrow \uparrow, \uparrow \uparrow} & \chi_{\uparrow \uparrow, \downarrow \downarrow} \\
\chi_{\downarrow \downarrow, \uparrow \uparrow} & \chi_{\downarrow \downarrow, \downarrow \downarrow}
\end{array}\right) \\
& \underline{\chi}_{T}(\mathbf{q}, \omega)=\left(\begin{array}{ll}
\chi_{\uparrow \downarrow, \uparrow \downarrow} & \chi_{\uparrow \downarrow, \downarrow \uparrow} \\
\chi_{\downarrow \uparrow, \uparrow \downarrow} & \chi_{\downarrow \uparrow, \downarrow \uparrow}
\end{array}\right),
\end{aligned}
$$

and similar for the interacting case. The transverse part of the interacting response function is diagonal, and can be expressed via TDDFT as

$$
\underline{\chi}_{T}^{\text {int }}(\mathbf{q}, \omega)=\left(\begin{array}{cc}
\frac{\chi_{\uparrow \downarrow, \uparrow \downarrow}}{1-\chi_{\uparrow \downarrow, \uparrow \downarrow} f_{\uparrow \downarrow, \uparrow \downarrow}^{\mathrm{xc}}} & 0 \\
0 & \frac{\chi_{\downarrow \uparrow, \downarrow \uparrow}}{1-\chi_{\downarrow \uparrow, \downarrow \uparrow} f_{\downarrow \uparrow, \downarrow \uparrow}^{\mathrm{xc}}}
\end{array}\right) .
$$

We now consider the case $\mathbf{q}=0$, where the spin-flip Lindhard functions have the 
simple form

$$
\begin{aligned}
\chi_{\uparrow \downarrow, \uparrow \downarrow}(0, \omega) & =-\frac{n \zeta}{\omega-Z^{*}} \\
\chi_{\downarrow \uparrow, \downarrow \uparrow}(0, \omega) & =\frac{n \zeta}{\omega+Z^{*}}
\end{aligned}
$$

(for a comprehensive discussion of the Lindhard function - the response function of the noninteracting electron gas - see Ref. [2]). We get a collective excitation at that frequency where $\underline{\chi}_{T}^{\text {int }}$ is singular. We substitute Eqs. (3.24) and (3.25) into Eq. (3.23) and set the determinant of the $2 \times 2$ transverse response matrix $\underline{\chi}_{T}^{\text {int }}$ to zero. Furthermore, because the system has no transverse spin polarization in the ground state, we have

$$
f_{\uparrow \downarrow, \uparrow \downarrow}^{\mathrm{xc}}(q, \omega)=f_{\downarrow \uparrow, \downarrow \uparrow}^{\mathrm{xc}}(q, \omega) \equiv f_{T}^{\mathrm{xc}}(q, \omega) .
$$

This yields the $\mathbf{q}=0$ limit of the spin-flip wave of the 2DEG as

$$
\omega_{\mathrm{sw}, 0}=Z^{*}-n \zeta f_{T}^{\mathrm{xc}}\left(0, \omega_{\mathrm{sw}, 0}\right)
$$

This expression is formally exact. Comparing with the many-body result (3.11), and using Eq. (3.20), gives

$$
f_{T}^{\mathrm{xc}}(0, Z)=\frac{2}{n \zeta} \frac{\partial e_{\mathrm{xc}}}{\partial \zeta}
$$

Equation (3.28) is an exact constraint on the transverse xc kernel of the 2DEG, based on Larmor's theorem. It is not difficult to show that it is satisfied by the adiabatic local-density approximation (ALDA), where the xc kernel is frequency- and momentum-independent [78, 82].

Larmor's theorem in the 2DEG can be understood from rather simple physical 
arguments [78]. A collective excitation of the spin-polarized ground state by coherently flipping all spins does not change the overall exchange energy of the 2DEG; hence, the $q=0$ spin-wave mode has no Coulomb contributions, and $\omega_{\mathrm{sw}, 0}=Z$. By contrast, flipping the spin of a single electron with respect to all other spins causes an exchange energy penalty; the energy difference between collective and single-particle excitation is $Z^{*}-Z>0$.

For small but finite wave vectors, one obtains the long-wavelength spin-flip wave dispersion [78]:

$$
\omega_{\mathrm{sw}, q}=Z-\frac{1}{|\zeta|} \frac{Z}{Z^{*}-Z} \frac{\hbar}{2 m} q^{2}
$$

which yields the spin-wave stiffness $S_{\mathrm{sw}}=-\frac{1}{|\zeta|} \frac{Z}{Z^{*}-Z} \frac{\hbar}{m}$, see Eq. (3.2). Interestingly, and in contrast with magnon dispersions in ferromagnetic systems, $S_{\mathrm{sw}}$ is negative, except for very low densities $\left(r_{s} \gtrsim 25\right)$. To understand this, we use the expression $e_{0}=\pi n\left(1+\zeta^{2}\right) / 2$ for the noninteracting kinetic energy per particle of a spin-polarized 2DEG [2], and recast the spin-wave stiffness as $S_{\mathrm{sw}}=\left(e_{\mathrm{xc}}^{\prime}+e_{0}^{\prime}\right) /|\zeta| e_{\mathrm{xc}}^{\prime}$, where the prime is a shorthand for $\partial / \partial \zeta . S_{\mathrm{sw}}$ thus has kinetic and xc contributions, which have opposite signs, except for the low-density limit in which the 2DEG becomes ferromagnetic. The xc contribution tends to increase the Coulomb energy as $q$ increases, since more spins become antiparallel; however, the kinetic energy contribution becomes more negative, and turns out to be the dominant one. Therefore, the spin-wave energy decreases with $q$. 


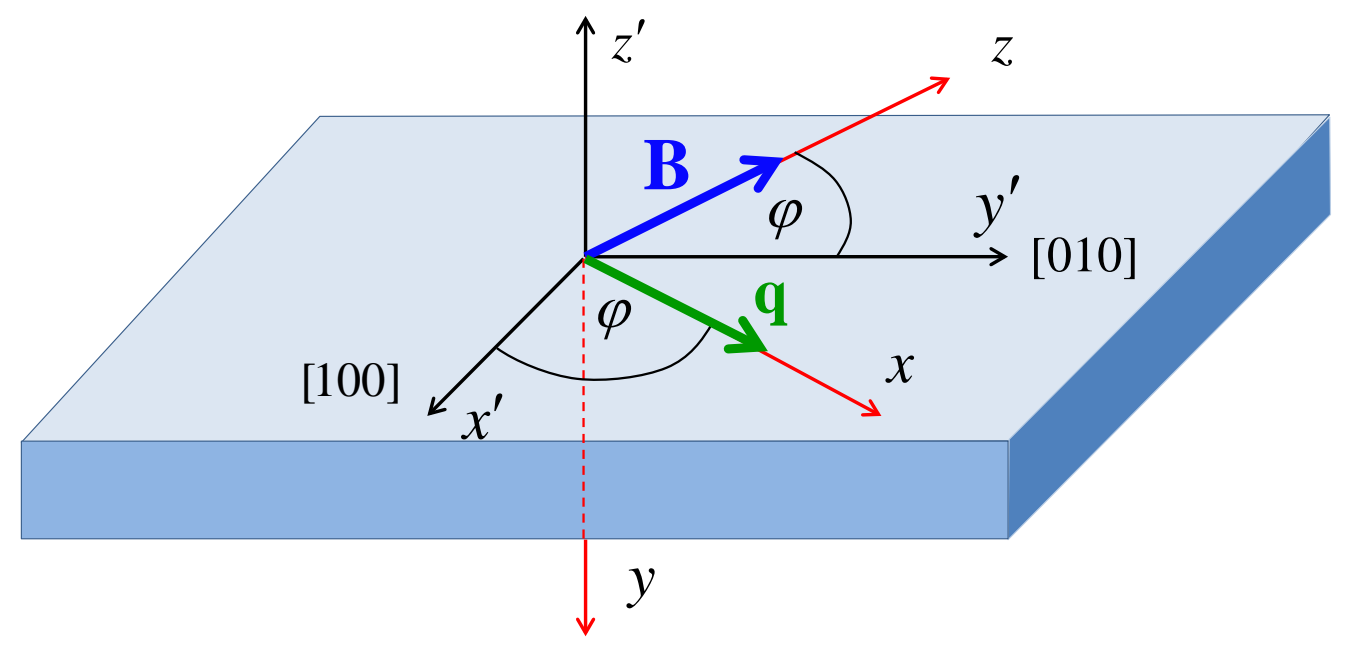

Figure 3.1: Reference frames $\mathcal{R}^{\prime}$ (black) and $\mathcal{R}$ (red) used to describe the electronic states in a quantum well with in-plane magnetic field $\mathbf{B}$ and spin-wave propagation direction $\mathbf{q}$.

\subsection{Quantum well with in-plane magnetic field and SOC}

In this Section we will consider the electronic ground state of an $n$-doped semiconductor quantum well with in-plane magnetic field and Rashba and Dresselhaus SOC, using DFT and the effective-mass approximation. The problem of interacting 2D electrons in the presence of SOC and external fields has been well-studied $[65,66,67,83,84,85,86,87,88,89]$; however, to our knowledge the results derived in this Section have not been given in the literature before.

The setup is illustrated in Figure 3.1, which defines two reference frames. The reference frame $\mathcal{R}^{\prime}$ is fixed with respect to the quantum well: the quasi-2DEG lies in the $x^{\prime}-y^{\prime}$ plane, where the $x^{\prime}$-axis points along the crystallographic [100] direction and the $y^{\prime}$-axis points along the [010] direction. The $z^{\prime}$-axis is along the direction of quantum confinement of the well. 
The coordinate system $\mathcal{R}$ is oriented such that its $x-z$ plane lies in the quantum well plane, and the z-axis points along the in-plane magnetic field $\mathbf{B}$. In the inelastic light scattering experiments that we will discuss below, $\mathbf{B}$ is always perpendicular to the wave vector $\mathbf{q}$ of the spin waves. Here, $\mathbf{q}$ is along the $x$-axis, which is at an angle $\varphi$ with respect to the $x^{\prime}$-axis.

The single-particle states in the reference frame $\mathcal{R}^{\prime}$ can be written as

$$
\Psi_{j \mathbf{k}}^{\prime}\left(\mathbf{r}^{\prime}\right)=e^{i \mathbf{k} \cdot \mathbf{r}^{\prime}} \psi_{j \mathbf{k}}^{\prime}\left(z^{\prime}\right)
$$

Here, $\mathbf{k}=\left(k_{x^{\prime}}, k_{y^{\prime}}, 0\right)$ is the in-plane wave vector and $j$ is the subband index; in the following, we are only interested in the lowest spin-split subband, so the subband index $j$ will be replaced by the index $p= \pm 1$. The two-component spinors $\psi_{p \mathbf{k}}^{\prime}\left(z^{\prime}\right)$ are obtained from the following Kohn-Sham equation:

$$
\left[h_{0} \hat{\sigma}_{0}+h_{x^{\prime}} \hat{\sigma}_{x^{\prime}}+h_{y^{\prime}} \hat{\sigma}_{y^{\prime}}\right] \psi_{p \mathbf{k}}^{\prime}\left(z^{\prime}\right)=E_{p \mathbf{k}} \psi_{p \mathbf{k}}^{\prime}\left(z^{\prime}\right)
$$

where $\hat{\sigma}_{0}$ is the $2 \times 2$ unit matrix. The spin-independent, diagonal part of the singleparticle Hamiltonian is

$$
h_{0}=\frac{k^{2}}{2}-\frac{1}{2} \frac{d^{2}}{d z^{\prime 2}}+v_{\mathrm{conf}}\left(z^{\prime}\right)+v_{\mathrm{H}}\left(z^{\prime}\right)+v_{\mathrm{xc}}^{+}\left(z^{\prime}\right) .
$$

Here, $v_{\text {conf }}\left(z^{\prime}\right)$ is the quantum well confining potential (an asymmetric square well), $v_{\mathrm{H}}\left(z^{\prime}\right)$ is the Hartree potential, and we define $v_{\mathrm{xc}}^{ \pm}\left(z^{\prime}\right)=\left[v_{\mathrm{xc} \uparrow}\left(z^{\prime}\right) \pm v_{\mathrm{xc} \downarrow}\left(z^{\prime}\right)\right] / 2$.

The off-diagonal parts in Eq. (3.31) contain the Zeeman energy $Z$ plus xc and 
SOC contributions:

$$
\begin{aligned}
& h_{x^{\prime}}=-\left(\frac{Z}{2}+v_{\mathrm{xc}}^{-}\left(z^{\prime}\right)\right) \sin \varphi+\alpha k_{y^{\prime}}+\beta k_{x^{\prime}} \\
& h_{y^{\prime}}=\left(\frac{Z}{2}+v_{\mathrm{xc}}^{-}\left(z^{\prime}\right)\right) \cos \varphi-\alpha k_{x^{\prime}}-\beta k_{y^{\prime}},
\end{aligned}
$$

where $\alpha$ and $\beta$ are the standard Rashba and Dresselhaus coupling parameters.

To find the solutions of the Kohn-Sham system, it is convenient to transform into the reference system $\mathcal{R}$ of Fig. 3.1, whose $z$-axis is along the magnetic field direction. We introduce two in-plane vectors, $\mathbf{q}_{0}$ and $\mathbf{q}_{1}$, whose components (in the frame $\mathcal{R}^{\prime}$ ) are

$$
\begin{aligned}
& q_{0 x^{\prime}}=2(\alpha \cos \varphi+\beta \sin \varphi) \\
& q_{0 y^{\prime}}=2(\alpha \sin \varphi+\beta \cos \varphi)
\end{aligned}
$$

and

$$
\begin{aligned}
& q_{1 x^{\prime}}=2(-\alpha \sin \varphi+\beta \cos \varphi) \\
& q_{1 y^{\prime}}=2(\alpha \cos \varphi-\beta \sin \varphi) .
\end{aligned}
$$

With this, Eq. (3.31) transforms into

$$
\left[h_{0} \hat{\sigma}_{0}+\left(\frac{Z-\mathbf{k} \cdot \mathbf{q}_{0}}{2}+v_{\mathrm{xc}}^{-}\right) \hat{\sigma}_{z}+\frac{\mathbf{k} \cdot \mathbf{q}_{1}}{2} \hat{\sigma}_{x}\right] \psi_{p \mathbf{k}}=E_{p \mathbf{k}} \psi_{p \mathbf{k}}
$$

(the scalar products $\mathbf{k} \cdot \mathbf{q}_{0}$ and $\mathbf{k} \cdot \mathbf{q}_{1}$ are invariant under this coordinate transforma- 
tion). The solutions of Eq. (3.39) can be written as follows:

$$
E_{p \mathbf{k}}=\frac{k^{2}}{2}+\frac{\varepsilon_{\uparrow}+\varepsilon_{\downarrow}}{2}+\frac{p}{2} \sqrt{\left(Z^{*}-\mathbf{k} \cdot \mathbf{q}_{0}\right)^{2}+\left(\mathbf{k} \cdot \mathbf{q}_{1}\right)^{2}},
$$

where $Z^{*}=\varepsilon_{\uparrow}-\varepsilon_{\downarrow}$ and $p= \pm 1$. The associated eigenfunctions are

$$
\begin{aligned}
& \psi_{+, \mathbf{k}}(y)=\frac{1}{\sqrt{1+b^{2}}}\left(\begin{array}{c}
1 \\
b
\end{array}\right) \phi(y) \\
& \psi_{-, \mathbf{k}}(y)=\frac{1}{\sqrt{1+b^{2}}}\left(\begin{array}{c}
-b \\
1
\end{array}\right) \phi(y)
\end{aligned}
$$

and

$$
b=\frac{1}{\mathbf{k} \cdot \mathbf{q}_{1}}\left[\sqrt{\left(Z^{*}-\mathbf{k} \cdot \mathbf{q}_{0}\right)^{2}+\left(\mathbf{k} \cdot \mathbf{q}_{1}\right)^{2}}-Z^{*}+\mathbf{k} \cdot \mathbf{q}_{0}\right]
$$

The solutions (3.40)-(3.43) have been expressed in terms of the solutions in the absence of SOC, $\varepsilon_{\uparrow, \downarrow}$ and $\phi(y)$, which follow from

$$
\left[h_{0}-\frac{k^{2}}{2} \pm\left(\frac{Z}{2}+v_{\mathrm{xc}}^{-}\right)\right] \phi_{\uparrow, \downarrow}=\varepsilon_{\uparrow, \downarrow} \phi_{\uparrow \downarrow} .
$$

The spin-up and spin-down envelope functions $\phi_{\uparrow}$ and $\phi_{\downarrow}$ are practically identical for the systems considered here, which allowed us to use $\phi_{\uparrow} \approx \phi_{\downarrow} \equiv \phi$ to express the solutions (3.41) and (3.42) in a relatively compact form. This implies that the dependence of $v_{\mathrm{xc}}^{-}\left(z^{\prime}\right)$ on $z^{\prime}$ can be neglected in Eq. (3.44).

Finally, let us expand the solutions (3.40)-(3.43) in powers of the SOC coefficients 
$\alpha$ and $\beta$. We obtain to second order in SOC

$$
E_{p \mathbf{k}}=\frac{k^{2}}{2}+\frac{\varepsilon_{\uparrow}+\varepsilon_{\downarrow}}{2}+\frac{p}{2}\left(Z^{*}-\mathbf{k} \cdot \mathbf{q}_{0}+\frac{\left(\mathbf{k} \cdot \mathbf{q}_{1}\right)^{2}}{2 Z^{*}}\right)
$$

and

$$
\begin{aligned}
& \psi_{+}(y)=\left(\begin{array}{c}
1-\frac{\left(\mathbf{k} \cdot \mathbf{q}_{1}\right)^{2}}{8 Z^{* 2}} \\
\frac{\mathbf{k} \cdot \mathbf{q}_{1}}{2 Z^{*}}+\frac{\left(\mathbf{k} \cdot \mathbf{q}_{0}\right)\left(\mathbf{k} \cdot \mathbf{q}_{1}\right)}{2 Z^{* 2}}
\end{array}\right) \phi(y) \\
& \psi_{-}(y)=\left(\begin{array}{c}
-\frac{\mathbf{k} \cdot \mathbf{q}_{1}}{2 Z^{*}}-\frac{\left(\mathbf{k} \cdot \mathbf{q}_{0}\right)\left(\mathbf{k} \cdot \mathbf{q}_{1}\right)}{2 Z^{* 2}} \\
1-\frac{\left(\mathbf{k} \cdot \mathbf{q}_{1}\right)^{2}}{8 Z^{* 2}}
\end{array}\right) \phi(y) .
\end{aligned}
$$

We illustrate the energy dispersion (3.40) of the lowest spin-split subband in Fig. 3.2. Here, we consider an asymmetrically doped CdMn quantum well of width $20 \mathrm{~nm}$ and electron density $2.6 \times 10^{11} \mathrm{~cm}^{-1}$. An applied magnetic field of $B=4.18 \mathrm{~T}$ leads to the bare and renormalized Zeeman energies $Z=0.40 \mathrm{meV}$ and $Z^{*}=0.573 \mathrm{meV}$, respectively, using the LDA. Here, we use the effective-mass parameters $m^{*}=0.105 m$, $e^{*}=1 / \sqrt{10}$, and $g^{*}=-1.64$ for CdTe.

We choose the Rashba and Dresselhaus parameters $\alpha=2.2 \mathrm{meV} \AA$ and $\beta=3.9$ meVA (see below), which causes the two subband to be slightly displaced horizontally with respect to one another (in Fig. 3.2, we plot $k$ along the [110] direction, i.e., for $\left.\varphi=45^{\circ}\right)$. 


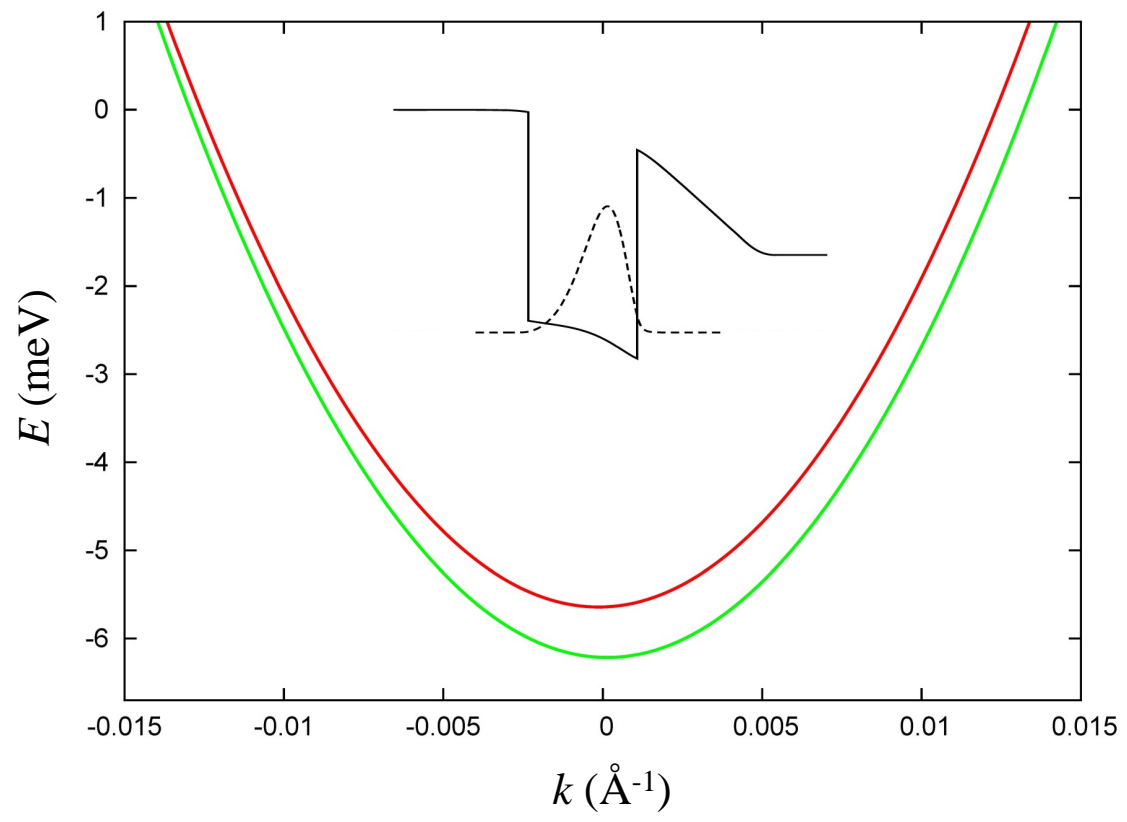

Figure 3.2: $\quad$ Spin-split lowest subband, Eq. (3.40), of an asymmetrically doped 20 nm CdTe quantum well with $B=4.18 \mathrm{~T}$, with $\alpha=2.2 \mathrm{meV} \AA$ and $\beta=3.9 \mathrm{meV} \AA$, taken at an angle $\varphi=45^{\circ}$ (i.e. along [110]). The inset shows the quantum well profile and the electronic density distribution.

\subsection{Spin-flip waves dispersion}

\subsubsection{Linear-response formalism}

In the following, we are interested in the collective spin-flip modes in a quantum well with in-plane magnetic field and SOC. Based on the translational symmetry in the $x-z$ plane, one can Fourier transform with respect to the in-plane position vector $\mathbf{r}=(x, z)$; this introduces the in-plane wave vector $\mathbf{q}$. The TDDFT linear-response equation (3.14) then becomes

$$
n_{\sigma \sigma^{\prime}}^{(1)}(\mathbf{q}, y, \omega)=\sum_{\tau \tau^{\prime}} \int d y^{\prime} \chi_{\sigma \sigma^{\prime}, \tau \tau^{\prime}}\left(\mathbf{q}, y, y^{\prime}, \omega\right) v_{\tau \tau^{\prime}}^{(1) \mathrm{eff}}\left(\mathbf{q}, y^{\prime}, \omega\right)
$$


where the noninteracting response function is given by

$$
\begin{aligned}
& \chi_{\sigma \sigma^{\prime}, \tau \tau^{\prime}}\left(\mathbf{q}, y, y^{\prime}, \omega\right)= \\
& -\sum_{p p^{\prime}}^{ \pm 1} \int \frac{d^{2} k}{(2 \pi)^{2}} \frac{\psi_{p \sigma}(\mathbf{k}, y) \psi_{p^{\prime} \sigma^{\prime}}^{*}(\mathbf{k}-\mathbf{q}, y) \psi_{p \tau}^{*}\left(\mathbf{k}, y^{\prime}\right) \psi_{p^{\prime} \tau^{\prime}}\left(\mathbf{k}-\mathbf{q}, y^{\prime}\right)}{\omega-E_{p \mathbf{k}}+E_{p^{\prime} \mathbf{k}-\mathbf{q}}+i \eta} \theta\left(E_{F}-E_{p \mathbf{k}}\right) \\
& +\sum_{p p^{\prime}}^{ \pm 1} \int \frac{d^{2} k}{(2 \pi)^{2}} \frac{\psi_{p^{\prime} \sigma}(\mathbf{k}+\mathbf{q}, y) \psi_{p \sigma^{\prime}}^{*}(\mathbf{k}, y) \psi_{p^{\prime} \tau}^{*}\left(\mathbf{k}+\mathbf{q}, y^{\prime}\right) \psi_{p \tau^{\prime}}\left(\mathbf{k}, y^{\prime}\right)}{\omega+E_{p \mathbf{k}}-E_{p^{\prime} \mathbf{k}+\mathbf{q}}+i \eta} \theta\left(E_{F}-E_{p \mathbf{k}}\right) .
\end{aligned}
$$

The energy eigenvalues $E_{p \mathbf{k}}$ and the single-particle states $\psi_{p \sigma}(\mathbf{k}, y)$ are defined in Eqs. (3.45)-(3.47). $\theta$ is the step function, and the Fermi energy is given by $E_{F}=$ $\pi N_{s}-\left(\alpha^{2}+\beta^{2}\right)$, where $N_{s}$ is the electronic sheet density (the number of electrons per unit area). We assume here that both spin-split subbands are occupied, which is different from the situation considered in Refs. [87, 88, 89].

In the response function (3.49) we only consider spin-flip excitations within the lowest spin-split subband of the quantum well; contributions from higher subbands are ignored, because they will be irrelevant as long as the Zeeman splitting is small compared to the separation between the lowest and higher subbands, which is safely the case here.

An interesting property of the response equation (3.48) is that it is invariant under the simultaneous sign changes $\alpha \rightarrow-\alpha, \beta \rightarrow-\beta$, and $\mathbf{q} \rightarrow-\mathbf{q}$, as can easily be seen from the form of the response function (3.49). From this we conclude that an expansion of the coefficients $E_{0}$ and $E_{2}$ in Eq. (3.4) only has even orders of $\alpha, \beta$, while only odd orders of $\alpha, \beta$ contribute to $E_{1}$.

The $4 \times 4$ matrix response equation (3.48) can be solved numerically, within the 
ALDA, to yield the spin-wave dispersions [68, 69]. However, much physical insight can be gained by an analytic treatment, which can be done for small wave vectors q: the spin-wave dispersion then takes on the form of Eq. (3.4), and our goal is to determine the coefficients $E_{0}$ and $E_{2}$ and compare them to experiment. We have done this analytically for $E_{0}$ and numerically for $E_{2}$, as discussed below.

Instead of the spin-density-matrix response (3.48), it is convenient to work with the density-magnetization response (especially for the calculations carried out in Appendix D, where we obtain corrections to second order in SOC): we replace the spindensity matrix $n_{\sigma \sigma^{\prime}}$, defined in Eq. (3.12), with the total density $n \equiv m_{0}$ and the three components of the magnetization $m_{x, y, z}$ as basic variables. In the following, we replace the labels $(x, y, z)$ with $(1,2,3)$ to streamline the notation.

The connection between the two sets of variables is made via the Pauli matrices:

$$
m_{i}(\mathbf{r})=\operatorname{tr}\left\{\hat{\sigma}_{i} \underline{\underline{n}}(\mathbf{r})\right\}, \quad i=0, \ldots, 3 .
$$

We can also express this through a $4 \times 4$ transformation matrix $\underline{\underline{T}}$, connecting the elements $m_{i}$ and $n_{\sigma \sigma^{\prime}}$ arranged as column vectors: $\vec{m}=\underline{\underline{T}} \vec{n}$. In detail,

$$
\left(\begin{array}{c}
m_{0} \\
m_{1} \\
m_{2} \\
m_{3}
\end{array}\right)=\left(\begin{array}{cccc}
1 & 0 & 0 & 1 \\
0 & 1 & 1 & 0 \\
0 & i & -i & 0 \\
1 & 0 & 0 & -1
\end{array}\right)\left(\begin{array}{c}
n_{\uparrow \uparrow} \\
n_{\uparrow \downarrow} \\
n_{\downarrow \uparrow} \\
n_{\downarrow \downarrow}
\end{array}\right) .
$$

In a similar way, one can transform the spin-density-matrix response equation (3.48) 
into the response equation for the density-magnetization:

$$
m_{i}^{(1)}(\mathbf{q}, y, \omega)=\sum_{k=0}^{3} \int d y^{\prime} \Pi_{i k}\left(\mathbf{q}, y, y^{\prime}, \omega\right) V_{k}^{(1)}\left(\mathbf{q}, y^{\prime}, \omega\right)
$$

where $\underline{\underline{\Pi}}=2 \underline{\underline{T}} \underline{\underline{\chi}} \underline{\underline{T}}^{-1}$ is the noninteracting density-magnetization response function, and $\vec{V}^{(1)}=\frac{1}{2} \underline{\underline{T}} \vec{v}^{(1) \text { eff }}$ is the effective perturbing potential.

We are only interested in the spin-flip excitations, which are eigenmodes of the system: hence, no external perturbation is necessary. Furthermore, the Hartree contributions drop out in the spin channel, so the effective potential only consists of the xc part:

$$
V_{k}^{(1)}(\mathbf{q}, y, \omega)=\sum_{l=0}^{3} \int d y^{\prime} h_{k l}^{\mathrm{xc}}\left(\mathbf{q}, y, y^{\prime}, \omega\right) m_{l}^{(1)}\left(\mathbf{q}, y^{\prime}, \omega\right) .
$$

In the ALDA, the xc kernels $h_{k l}^{\mathrm{xc}}$ do not depend on frequency and wave vector [68]. Once we have the density-magnetization response, we can multiply it with the xc matrix. The xc matrix has a simple form, because in this reference frame the spin polarization direction is along $z$ :

$$
\underline{\underline{H}}^{\mathrm{xc}}=\left(\begin{array}{cccc}
h_{00}^{\mathrm{xc}} & 0 & 0 & h_{03}^{\mathrm{xc}} \\
0 & h_{11}^{\mathrm{xc}} & 0 & 0 \\
0 & 0 & h_{22}^{\mathrm{xc}} & 0 \\
h_{30}^{\mathrm{xc}} & 0 & 0 & h_{33}^{\mathrm{xc}}
\end{array}\right)
$$


where

$$
\begin{aligned}
h_{00}^{\mathrm{xc}} & =2 \frac{\partial e_{\mathrm{xc}}}{\partial n}+n \frac{\partial^{2} e_{\mathrm{xc}}}{\partial n^{2}}-2 \zeta \frac{\partial^{2} e_{\mathrm{xc}}}{\partial n \partial \zeta}+\frac{\zeta^{2}}{n} \frac{\partial^{2} e_{\mathrm{xc}}}{\partial n^{2}} \\
h_{03}^{\mathrm{xc}} & =h_{30}^{\mathrm{xc}}=\frac{\partial^{2} e_{\mathrm{xc}}}{\partial n \partial \zeta}-\frac{\zeta}{n} \frac{\partial^{2} e_{\mathrm{xc}}}{\partial \zeta^{2}} \\
h_{11}^{\mathrm{xc}} & =h_{22}^{\mathrm{xc}}=\frac{1}{n \zeta} \frac{\partial e_{\mathrm{xc}}}{\partial \zeta} \\
h_{33}^{\mathrm{xc}} & =\frac{1}{n} \frac{\partial^{2} e_{\mathrm{xc}}}{\partial \zeta^{2}} .
\end{aligned}
$$

All quantities are evaluated at the local density $n(y)$ and spin polarization $\zeta(y)$ and multiplied with $\delta\left(y-y^{\prime}\right)$. Here, $e_{\mathrm{xc}}$ is the xc energy per particle of the 3D electron gas $[90]$.

To find the collective modes, we can recast the response equation (3.52) in such a way that the $y$-dependence goes away; the xc kernels $h_{k l}^{\mathrm{xc}}$ are then replaced by their averages over $\phi^{4}(y)$. We need to determine those frequencies where the matrix

$$
\underline{\underline{M}}(\mathbf{q}, \omega)=\underline{\underline{H}}^{\mathrm{xc}}(\mathbf{q}, \omega) \underline{\underline{\Pi}}(\mathbf{q}, \omega)
$$

has the eigenvalue 1 . In other words, we solve the $4 \times 4$ eigenvalue problem

$$
\underline{\underline{M}}(\mathbf{q}, \omega) \vec{x}=\lambda(\mathbf{q}, \omega) \vec{x}
$$

and find the mode frequencies by solving $\lambda(\mathbf{q}, \omega)=1$ for $\omega$, where $\mathbf{q}$ is fixed. In general there will be 4 solutions. This is in principle exact, provided we know the exact Hxc matrix, which, in general, depends on $(\mathbf{q}, \omega)$. In ALDA, it is a constant (for given density and spin polarization). 


\subsubsection{Beyond Larmor's theorem: leading SOC corrections}

In the presence of SOC, the spin-wave dispersions are modified in an interesting and subtle manner. For small values of $\mathbf{q}$, the spin-wave dispersion has the quadratic form given in Eq. (3.4). Our goal is now to obtain the coefficient $E_{0}$ to leading order in the Rashba and Dresselhaus coupling strengths $\alpha$ and $\beta$. To do this, we carry out a perturbative expansion of the eigenvalue problem (3.60) in orders of SOC. At $q=0$, the matrix can be written as

$$
\underline{\underline{M}}(0, \omega)=\underline{\underline{M}}^{(0)}+\underline{\underline{M}}^{(2)}+\ldots
$$

where superscripts indicate the order of SOC (the linear order vanishes at $q=0$ ).

We first solve the zero-order eigenvalue problem $\underline{\underline{M}}^{(0)} \vec{x}^{(0)}=\lambda^{(0)} \vec{x}^{(0)}$. The zeroorder spin-flip response function is

$$
\underline{\underline{\Pi}}^{(0)}\left(0, y, y^{\prime}, \omega\right)=\frac{Z^{*} \phi^{2}(y) \phi^{2}\left(y^{\prime}\right)}{\pi\left(\omega^{2}-Z^{*^{2}}\right)}\left(\begin{array}{cccc}
0 & 0 & 0 & 0 \\
0 & Z^{*} & -i \omega & 0 \\
0 & i \omega & Z^{*} & 0 \\
0 & 0 & 0 & 0
\end{array}\right) \text {. }
$$

Defining

$$
f_{T}=\left.\int d y \frac{\phi^{4}(y)}{\pi n(y) \zeta(y)} \frac{\partial e_{\mathrm{xc}}}{\partial \zeta}\right|_{n(y), \zeta(y)}
$$


where $f_{T}<0$, we obtain

$$
\underline{\underline{M}}^{(0)}=\frac{Z^{*} f_{T}}{\omega^{2}-Z^{* 2}}\left(\begin{array}{cccc}
0 & 0 & 0 & 0 \\
0 & Z^{*} & -i \omega & 0 \\
0 & i \omega & Z^{*} & 0 \\
0 & 0 & 0 & 0
\end{array}\right)
$$

This matrix has eigenvalue 1 for

$$
\omega=Z^{*}+Z^{*} f_{T}=Z
$$

(we discard the negative-frequency solution) in accordance with Larmor's theorem. The associated eigenvector is $\vec{x}^{(0)}=2^{-1 / 2}(0,-i, 1,0)$.

To obtain the change of the collective spin precession caused by the presence of SOC, we need to determine $\lambda^{(2)}$. Using perturbation theory we obtain the secondorder correction of the eigenvalue as

$$
\lambda^{(2)}=\left[\vec{x}^{(0)}\right]^{\dagger} \underline{\underline{M}}^{(2)} \vec{x}^{(0)}
$$

To construct $\underline{\underline{M}}^{(2)}$ we need $\underline{\underline{\Pi}}^{(2)}(0, \omega)$, the spin-flip response matrix expanded to second order in $\alpha$ and $\beta$, which requires a rather lengthy calculation (see Appendix D). We end up with

$$
\lambda^{(2)}=\frac{2 \pi N_{s}}{Z^{* 2} f_{T}^{2}}\left[\left(\alpha^{2}+\beta^{2}\right)\left(3 f_{T}+2\right)+2 \alpha \beta \sin (2 \varphi)\left(f_{T}+2\right)\right]
$$

The condition $1=\lambda^{(0)}+\lambda^{(2)}$ gives the final result for $E_{0}$, see Eq. (3.6). 
Let us now turn to the other two coefficients in Eq. (3.4). The leading contribution to the linear coefficient $E_{1}$ is in first order in $\alpha$ and $\beta$, see Eq. (3.5), and was already obtained in Ref. [74]. The quadratic coefficient $E_{2}$ describes the renormalization of the spin-wave stiffness $S_{\mathrm{sw}}$ due to SOC. We did not attempt to derive an analytical expression for $E_{2}$, as it was done without SOC in Eq. (3.29), although this could in principle (and with much effort) be done along the same lines as for $E_{0}$. Instead, we extract $E_{2}$ from a fully numerical solution of the linear-response equation for the spin waves, which includes all orders of $\alpha$ and $\beta$.

\subsection{Results and discussion}

According to the theory presented above, the spin-flip excitations in a 2DEG in the presence of SOC depend on the direction of the applied magnetic field (direction $z$ in Fig. 3.1). Figure 3.3 depicts the spin-excitation spectra for $\varphi=45^{\circ}$ and $\varphi=135^{\circ}$, calculated using ALDA, for the same quantum well as in Fig. 3.2. Clearly, the spinwave dispersions and single-particle spin-flip continua differ drastically, depending on the direction of the in-plane momentum. In the following, we will compare our theory with experiment.

\subsubsection{Electronic Raman scattering}

We now describe the experimental work done by our collaborators in Paris: Electronic Raman scattering was used, whereby a well-controlled in-plane momentum $\mathbf{q}$ is transferred to the spin excitations of the 2DEG. Under the quasi-scattering geometry shown

in Fig. 2.4a, the transferred momentum is given by $\mathbf{q}=\boldsymbol{\kappa}_{i, \|}-\boldsymbol{\kappa}_{s, \|} \simeq \frac{4 \pi}{\lambda} \sin \theta \boldsymbol{e}_{x}$, where 

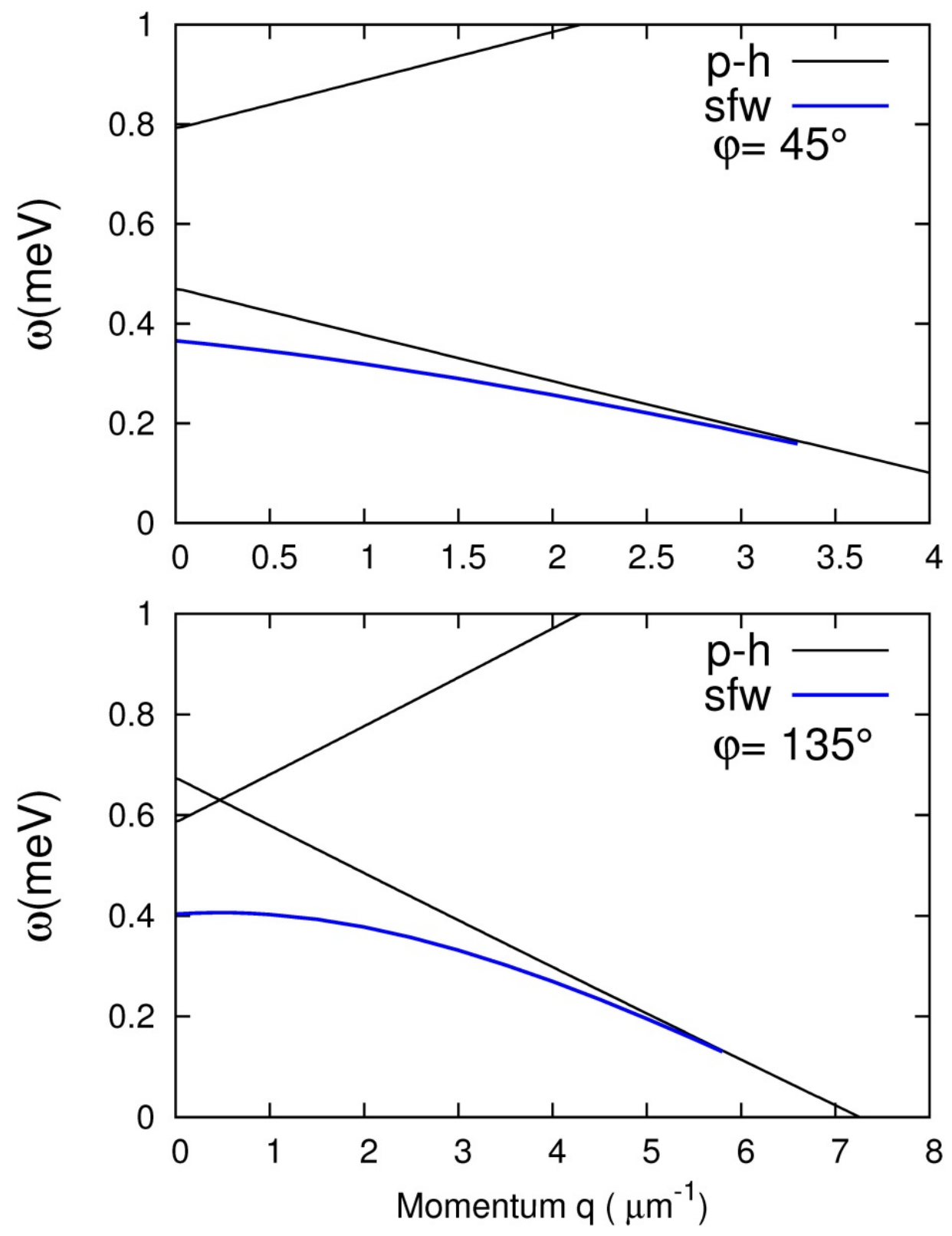

Figure 3.3: Spin-flip excitation spectra with SOC for $\varphi=45^{\circ}$ and $\varphi=135^{\circ}$, calculated using the ALDA for the same quantum well as in Fig. 3.2. Solid black lines: boundaries of the single-particle spin-flip continuum. Blue dashed lines: spin-wave dispersions. 
$\boldsymbol{\kappa}_{i}$ and $\boldsymbol{\kappa}_{s}$ are the wave vectors of the linearly cross-polarized incoming and scattered photons, and $\lambda$ is the incoming wavelength. Our setup allows us to vary $\mathbf{q}$ both in magnitude and in-plane orientation, while the magnetic field $\mathbf{B}_{\text {ext }}$ is applied in the plane of the well and always perpendicular to $\mathbf{q}$.

Our sample is an asymmetrically modulation-doped, 20 nm-thick $\mathrm{Cd}_{1-x} \mathrm{Mn}_{x} \mathrm{Te}$ $(x \simeq 0.13 \%)$ quantum well, grown along the [001] direction by molecular beam epitaxy, and immersed in a superfluid helium bath at temperature $2 \mathrm{~K}$. The density of the electron gas is $N_{s}=2.6 \times 10^{11} \mathrm{~cm}^{-2}$ and the mobility is $1.7 \times 10^{5} \mathrm{~cm}^{2} \mathrm{~V}^{-1} \mathrm{~s}^{-1}$. The small concentration of $\mathrm{Mn}$ introduces localized magnetic moments into the quantum well, which are polarized by the external $B$-field, and act to amplify it $[81,91]$.

Figure $3.4 \mathrm{~b}$ shows a series of spin-wave Raman lines obtained at fixed $B_{\text {ext }}=2 \mathrm{~T}$ and $q=0$, and for various in-plane angles $\varphi$. A clear modulation of the spin-wave energy with $\varphi$ was observed, evidencing the above predicted breakdown of Larmor's theorem.

To better understand the phenomenon, the full spin-wave dispersion by varying the transferred momentum $q$ was measured. Fig. 3.4c shows the dispersions for three different values of $\varphi$ : they exhibit a quadratic dependence with $q$, with a maximum shifted from the zone center. This shift from the zone center is well understood in the frame of the spin-orbit twist model [74]: SOC produces a rigid shift of the spin-wave dispersion by a momentum $-\mathbf{q}_{\mathbf{0}}$, see Eq. (3.35), which depends on $\varphi$. This produces the linear term in $q$ in the energy dispersion of Eq. (3.4).

The spin-wave dispersions for angles $\varphi$ between zero and $360^{\circ}$ have systematically been measured; for each angle, the data are fit to a parabola (as in Fig. 3.4c), which allows us to extract the coefficients $E_{0,1,2}(\varphi)$. The experimental results are shown in 
a
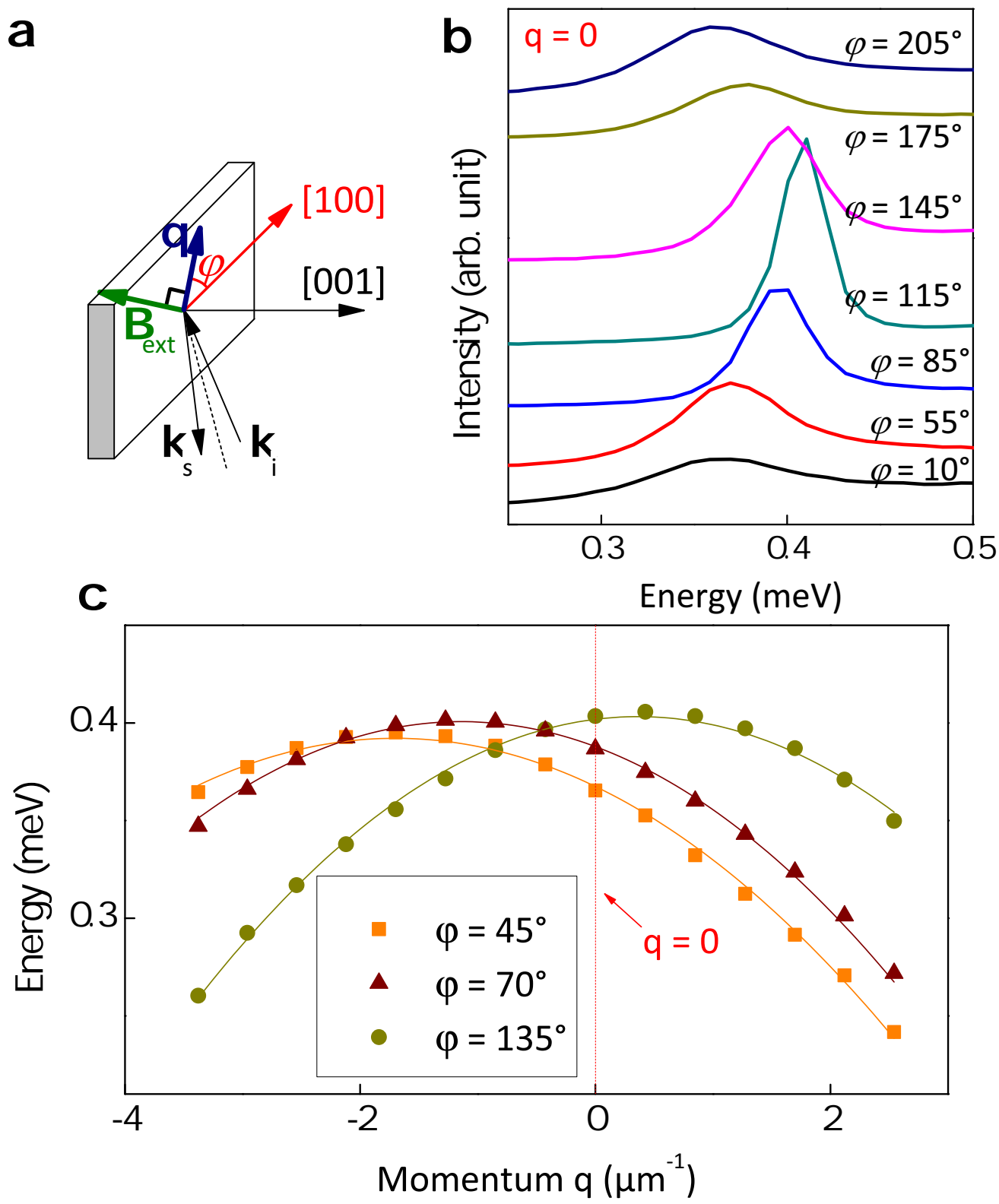

Figure 3.4: (a) Electronic Raman scattering geometry: $\boldsymbol{k}_{i}$ and $\boldsymbol{k}_{s}$ are the incoming and scattered light wave vectors, respectively; $\mathbf{q}$ is the transferred momentum, of in-plane orientation measured by the angle $\varphi$ from [100]. An external magnetic field $\mathbf{B}_{\text {ext }}$ is applied perpendicularly to $\mathbf{q}$. (b) Raman spectra of the spin wave, obtained at $B_{\text {ext }}=2 \mathrm{~T}$ and $q=0$, for a series of in-plane angles $\varphi$. (c) Momentum dispersion of the spin wave for different in-plane angles. 
both Figs. 3.5 and 3.6 (dots), clearly exhibiting the predicted sinusoidal modulations.

The modulation of $E_{0}$, with a relative amplitude of about $6 \%$, demonstrates the breakdown of Larmor's theorem. This effect is of second order in the SOC. By contrast, the modulation of $E_{1}$ is a first-order SOC effect. Another second-order SOC effect is the modulation of the curvature of the spin-wave dispersion, i.e. the spin-wave stiffness $S_{\mathrm{sw}}$. The bottom panels of Figs. 3.5 and 3.6 show the curvature $E_{2}=S_{\mathrm{sw}} / 2$ as a function the in-plane angle $\varphi$. Again, a sinusoidal variation is observed, with a relative amplitude of about $10 \%$; the phase of the modulation is opposite to that of $E_{0}$ and $E_{1}$.

\subsubsection{Comparison with theory}

In Figures 3.5 and 3.6, the experimental data for $E_{0}(\varphi), E_{1}(\varphi)$, and $E_{2}(\varphi)$ is compared with theory (lines). In our calculations, we consider, as before, a CdTe quantum well of width $20 \mathrm{~nm}$ and density $N_{s}=2.6 \times 10^{11} \mathrm{~cm}^{-2}$. The value of bare Zeeman splitting $Z$ is extracted from the data as follows. According to Eq. (3.6), $E_{0}$ can be written in the form $E_{0}(\varphi)=Z-a-b \sin (2 \varphi)$. For the range of input parameters $\alpha, \beta, Z$ and $Z^{*}$ under consideration (see below), the ratio $b / a \approx 1.5$ is almost constant. We temporary fix this ratio, and a fit with the data from the top panel of Fig. 3.5 then yields $Z=0.40 \mathrm{meV}$ and $b=0.024 \mathrm{meV}$ to within about $3 \mu \mathrm{eV}$. We can then calculate $Z^{*}$ using the ALDA xc kernel [see Eq. (3.65)], where $Z_{\mathrm{ALDA}}^{*}=Z /\left(1+f_{T}\right)=0.573$ meV. Now fixing $Z, Z^{*}$ and letting $b / a=1.5$, we fit $\alpha$ and $\beta$ from $E_{0}(\varphi)$ and $E_{1}(\varphi)$. An optimal agreement with the experimental results for $E_{0}$ and $E_{1}$ is achieved with $\alpha=1.6 \mathrm{meV} \AA$ and $\beta=3.1 \mathrm{meV} \AA$ [92].

Having determined the set of parameters $Z, Z^{*}, \alpha$ and $\beta$, we run the fully nu- 

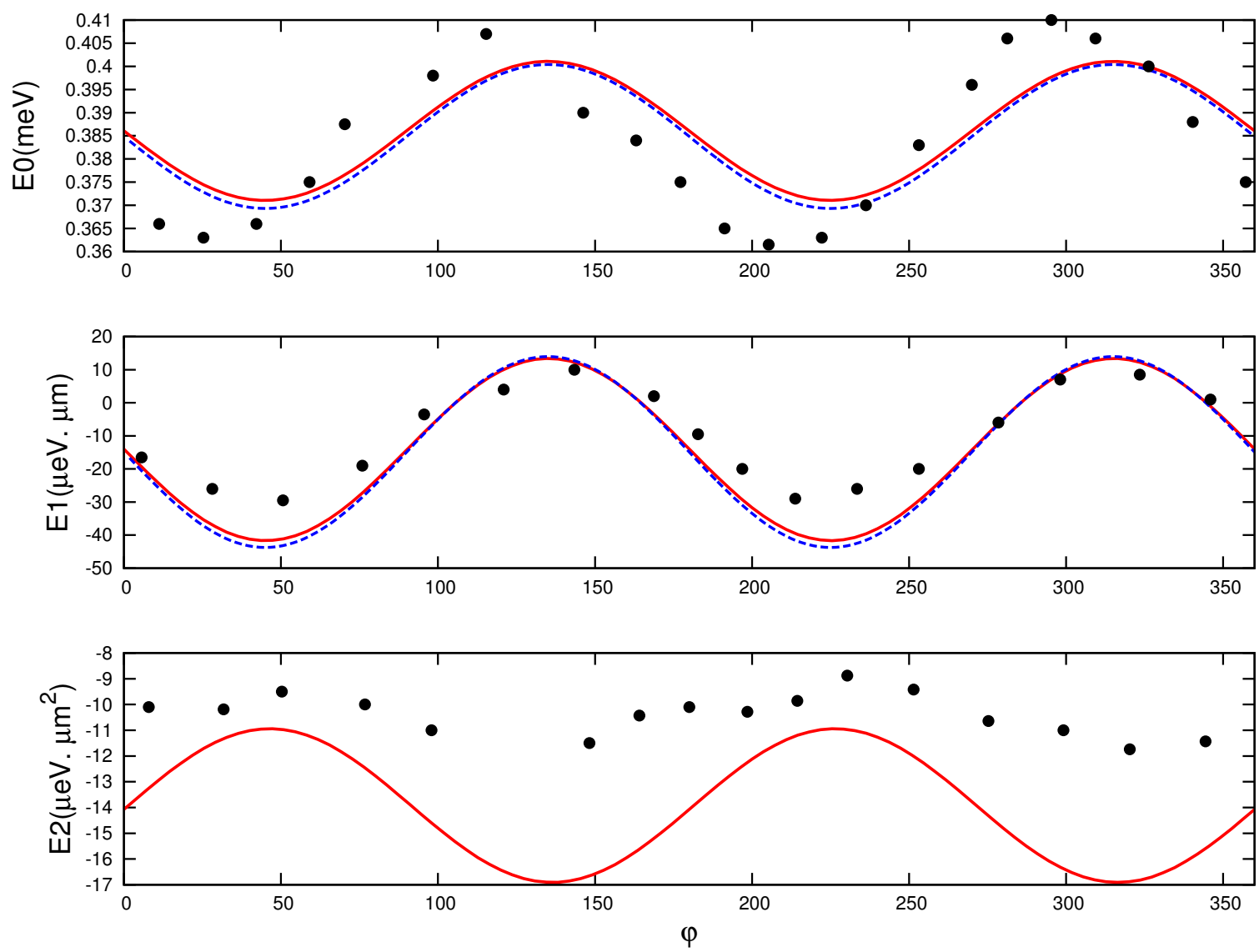

Figure 3.5: Coefficients $E_{0}, E_{1}$, and $E_{2}$ of the spin-wave dispersion, Eq. (3.4), as a function of angle $\varphi$. Dots: experimental data. Lines: theoretical results using $Z^{*}=0.573 \mathrm{meV}$ obtained with ALDA, and $\alpha=1.6 \mathrm{meV} \AA$ and $\beta=3.1 \mathrm{meV} \AA$ obtained by fitting $E_{0}$ and $E_{1}$. The red lines follow from the fully numerical solution of Eq. (3.60), the dashed blue lines follow from the analytical formulas (3.6) and $(3.5)$. 

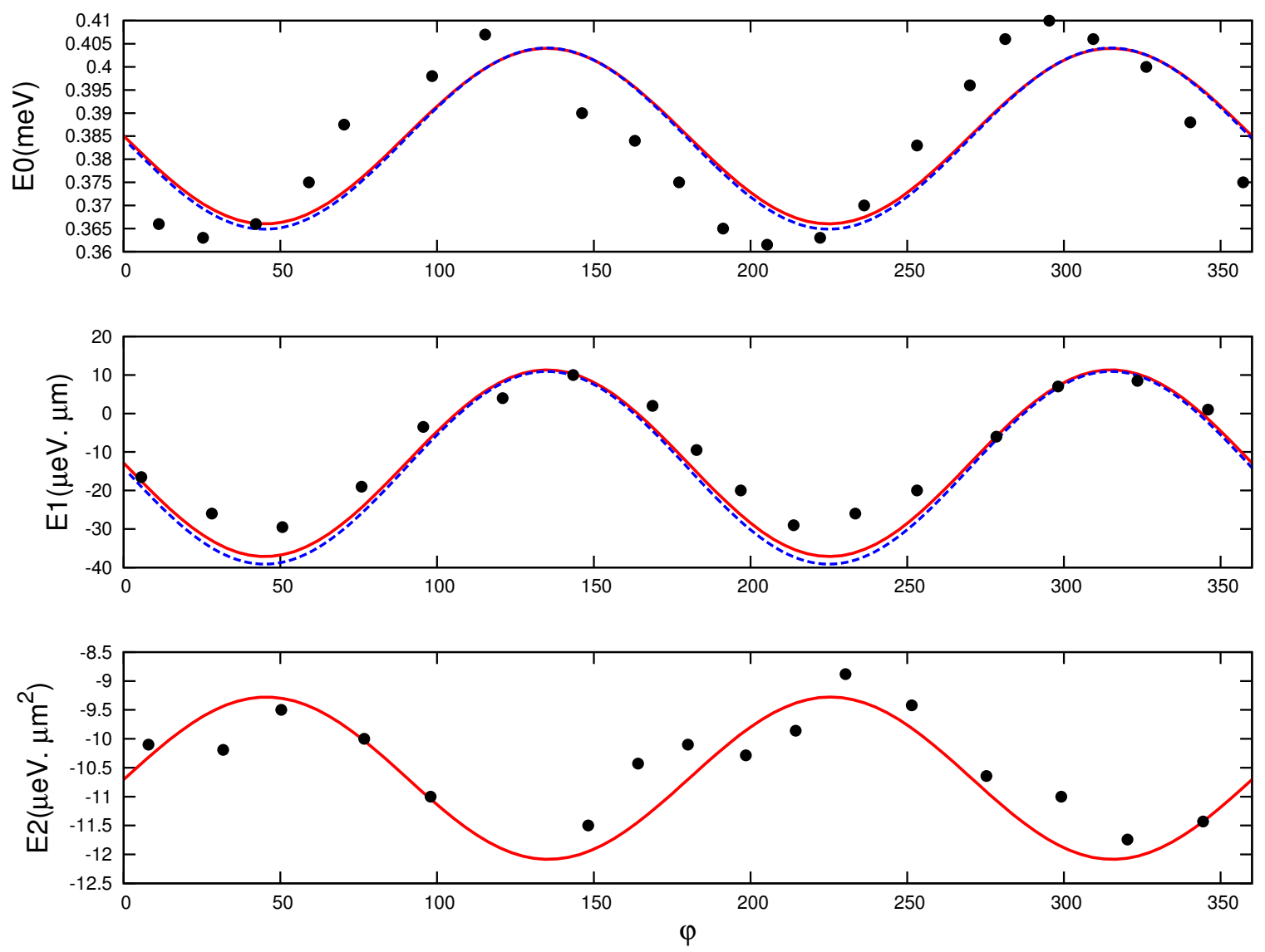

Figure 3.6: Same as Fig. 3.5, but using $Z^{*}=0.63 \mathrm{meV}, \alpha=2.2 \mathrm{meV} \AA$, and $\beta=3.9$ meVÅ obtained from a best fit to the experimental data. 
merical solution of the linear-response equation (3.60) for the spin-flip waves, and fit the small- $q$ dispersion to a parabola for a given angle $\varphi$ to extract $E_{0}, E_{1}$, and $E_{2}$. As shown in Fig. 3.5, both the analytical formulas of Eqs. (3.5) and (3.6) and the numerical solutions (the dashed blue and solid red lines, respectively) are in very good agreement with the experimental data for $E_{0}$ and $E_{1}$, apart from a shift in the phase of the experimental modulation of $E_{0}$, which is not accounted for by the theory.

An additional observation from Fig. 3.5 is that the analytical formulas and the numerical results for $E_{0}$ and $E_{1}$ are extremely close to each other. This is not surprising, since the next higher-order corrections to $E_{0}$ and $E_{1}$ are of fourth and third order in $\alpha, \beta$, respectively (as we showed in Section 3.3.1), and hence negligible.

On the other hand, the bottom panel of Fig. 3.5 shows that the calculation dramatically fails to reproduce $E_{2}$. Therefore, we repeated the calculations, but now using a renormalized Zeeman energy $Z^{*}$ that does not follow from the ALDA, but from a numerical fit. We fit the numerical solutions with $Z^{*}, \alpha$ and $\beta$ and then find that using $\alpha=2.2 \mathrm{meV} \AA, \beta=3.9 \mathrm{meV} \AA$ and $Z_{\text {fit }}^{*}=0.63 \mathrm{meV}$ we obtain an excellent agreement with the experimental results for all three modulation parameters, $E_{0}, E_{1}$, and $E_{2}$, as shown in Fig. 3.6.

The comparison between theory and experiment of the spin-wave modulation parameters thus demonstrates that the ALDA underestimates $Z^{*}$ by about $10 \%$, which seems to be a relatively minor deviation. However, $E_{0}, E_{1}$, and $E_{2}$ depend very sensitively on $Z^{*}$, which suggests a need for a more accurate description of dynamical xc effects beyond the ALDA.

We finally mention that additional contributions to the angular modulation of the spin-wave dispersion could arise from an in-plane anisotropy of the g-factor of the 
form $g_{x y} \sin (2 \varphi)$, where $g_{x y}$ is the off-diagonal component of the $g$ tensor $[93,94,95]$. However, by slightly varying the applied magnetic field around $2 \mathrm{~T}$, we have found that this effect contributes less that $15 \%$ of the modulations amplitude of $E_{0}$, and leads only to $\sim 7 \%$ changes of the parameters $\alpha$ and $\beta$ used to fit the data in Figs. 3.5 and 3.6; details are given in Appendix E.

\subsubsection{Density dependence of $E_{0}$}

To further test our theoretical prediction for the breakdown of Larmor's theorem [Eq. (3.6)], we will now explore the density dependence of the parameter $E_{0}$. In order to vary the electronic density in our sample, An additional continuous-wave green laser beam $(514.5 \mathrm{~nm})$ on the quantum well was shone. This illumination is above the band gap and generates electron-hole pairs in the barrier layer: the electrons neutralize some donor elements of the doping plane, while the holes migrate to the quantum well where they capture free electrons. This leads to a depopulation of the electron gas, which can be precisely controlled by the power of the above-gap illumination [72]. Using this technique, the density in our sample can be reproducibly reduced by up to a factor 2. $E_{0}(\varphi)$ for different values of $N_{s}$ was measured, and in Fig. 3.7 the amplitude of the $q=0$ modulation (solid circles), $\Delta E_{0}=\left(\operatorname{Max} E_{0}-\operatorname{Min} E_{0}\right) / 2$, as a function of the electron density was plotted.

Again, the data is well reproduced by the analytical result of Eq. (3.6) (blue line). The red circle represents the amplitude of $E_{0}$ for the reference density $N_{s}^{\text {ref }}=$ $2.6 \times 10^{11} \mathrm{~cm}^{-2}$, obtained from our numerical fit in the top panel of Fig. 3.6. To 


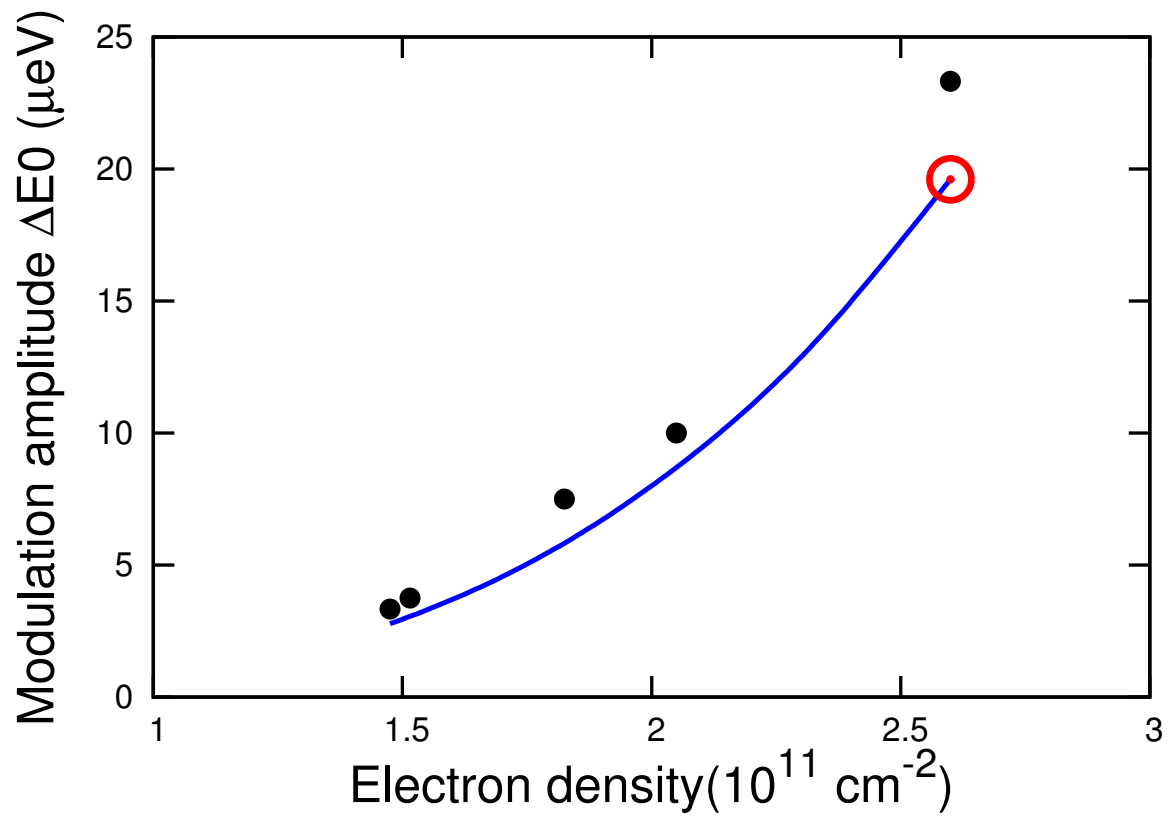

Figure 3.7: Amplitude of the modulation of the $q=0$ spin-wave energy, $\Delta E_{0}=$ $\left(\operatorname{Max} E_{0}-\operatorname{Min} E_{0}\right) / 2$, as a function of the sheet density $N_{s}$ of the electron gas in the quantum well. Black dots: experimental data. Blue line: analytical results using Eq. (3.6).

generate the blue line, we need $Z^{*}$ as a function of $N_{s}$, which we approximate as

$$
Z^{*}\left(N_{s}\right) \approx Z_{\mathrm{fit}}^{*}\left(N_{s}^{\mathrm{ref}}\right) \frac{Z_{\mathrm{ALDA}}^{*}\left(N_{s}\right)}{Z_{\mathrm{ALDA}}^{*}\left(N_{s}^{\mathrm{ref}}\right)}=1.10 Z_{\mathrm{ALDA}}^{*}\left(N_{s}\right)
$$

i.e., we approximate the density scaling using the ALDA. We also need the density dependence of the Rashba and Dresselhaus parameters $\alpha, \beta$. We approximate their density scaling using the $\mathbf{k} \cdot \mathbf{p}$ results of Ref. [72]. Both approximations are well justified by the excellent agreement between theory and experiment in Fig. 3.7. 


\subsection{Conclusions}

In this chapter, we presented a detailed theoretical and experimental study of spinwave dispersions in a 2DEG in the presence of Rashba and Dresselhaus SOC. In earlier work [74] we had limited ourselves to the leading (first-order) SOC effects, which causes a momentum-dependent shift of the spin-wave dispersions, but leaves the spin-wave stiffness as well as Larmor's theorem intact. We have now discovered some subtle corrections which arise when second-order SOC effects are taken into account: Larmor's theorem is broken, and the spin-wave stiffness is modified. Both corrections are relatively small (of order $10 \%$ or less) but experimentally detectable.

We presented a linear-response theory, based on TDDFT, to fully account for SOC effects to first, second and higher orders in SOC. A detailed comparison with experimental data, obtained using inelastic light scattering, confirmed the accuracy of the theory and allowed us to extract the SOC parameters $\alpha$ and $\beta$, as well as the renormalized Zeeman splitting $Z^{*}$.

A major outcome of our study is that we discovered that the ALDA does not lead to a satisfactory description of the second-order SOC modulation effects of the spin waves. At present, there are only few approaches in ground-state DFT for noncollinear magnetism that go beyond the LDA, such as the optimized effective potential (OEP) [96] or gradient corrections [97, 98, 99]. This provides motivation for the search for better xc functionals in TDDFT for noncollinear spins. In particular, as we mentioned in chapter 2, any such new xc functional should be well-behaved in the crossover between three-and two-dimensional systems.

The study of spin waves in electron gases confined in semiconductor quantum wells under the presence of SOC is also of practical interest. Manipulation of the 
Rashba and Dresselhaus coupling strengths can be used to control the spin-wave group velocity [74]. Since spin waves can be used as carriers of spin-based information, this may lead to applications in spintronics. Here we have provided a suitable theoretical framework to describe these effects. 


\section{Chapter 4}

\section{Spin-helix Larmor mode}

Spin-flip waves are collective excitations of magnetic systems [100, 101]: rather than flipping individual magnetic moments, which causes a large exchange energy penalty, the periodic reversal of magnetic moments extends as a precessional wave over the entire system, which is energetically favorable. There has been recent interest in spin waves in ferromagnetic thin films as an information carrier, which constitutes the basis for magnonics $[13,14]$ : Spin waves exist in systems with localized and itinerant magnetic moments. In the latter case, the precession of the interacting spins, the charge motion, and the spin-orbit coupling (SOC) due to inversion asymmetry are all interrelated and lead to novel phenomena. For example, chiral spin waves have been observed in asymmetric monolayers of iron [102, 103], helical spin waves have been predicted in a two-dimensional electron gas (2DEG) subject to Rashba SOC [87], and twisted spin waves have been predicted and observed in magnetized 2DEGs [74].

The fundamental and practical aspects of spin waves in the presence of SOC have drawn interest recently in the context of spintronics $[5,6,7,104]$. SOC provides 
the conversion of charge based information into the spin wave [15, 105]. However, the presence of both SOC and Coulomb interaction still poses interesting challenges, especially in the dynamical regime.

In this chapter, we will study spin waves in a $2 \mathrm{DEG}$ in the presence of in-plane magnetization and SOC. This system exhibits a rich interplay between Coulomb many-body effects, Rashba and Dresselhaus SOC, applied magnetic field, and electron density, which we have studied in chapter three and in several earlier publications by our group $[71,72,73,74]$. What we found is that the spin waves are modified by the SOC in a subtle manner: the spin waves get a boost of their group velocity whose magnitude and orientation depends on the crystallographic propagation direction in the quantum well plane. This interesting behavior of the spin waves can be understood via a transformation into a spin-orbit twisted reference frame; however, in general this only holds to lowest order in SOC [74].

In this chapter, we now consider a very special case in which exact results can be proved to all orders in SOC, namely, the case of a persistent spin helix $[106,107,108$, 109, 110, 111, 112, 113, 114]. The spin helix arises in a 2DEG in which the strengths of the Rashba and Dresselhaus fields, $\alpha$ and $\beta$, are equal (we here consider a 2DEG embedded in a zincblende quantum well grown along the [001] direction). SU(2) symmetry is then partially restored, and a helical spin texture can be sustained along the [110] direction. The main experimental signature of this state is that spin packet excitations are protected from decoherence, leading to extraordinarily long lifetimes $[109,112]$

If a magnetic field is applied in the plane of a 2DEG with $\alpha=\beta$, spin-packet excitations can sustain long-lived precessional motion [110]. Furthermore, some in- 
teresting magnetoelectronic effects can occur in 2DEGs $[115,116,117]$ or quantum

wires $[118,119]$ which reflect the special condition $\alpha=\beta$. However, to our knowledge the spin-wave dynamics under these circumstances, which involves Coulomb interactions between the electrons, has not been explicitly addressed before.

Our treatment of spin waves is based both on time-dependent density-functional theory (TDDFT) in the linear-response regime [29] and on an equation-of-motion approach featuring the full many-body Hamiltonian [74, 79]. We derive the exact form of the spin-wave dispersion for systems with a spin-helix texture in the absence of magnetic fields, and find that it is obtained from the dispersion without SOC by a simple wave vector shift. The spin-wave stiffness $S_{\mathrm{sw}}$ remains unchanged.

The main result of this chapter is that we identify an exact dynamical state of the 2DEG with $\alpha=\beta$, which can be characterized as a collective precession of the 2DEG about the spin-helix state. The precession occurs at the bare Zeeman frequency, and we therefore refer to it as the spin-helix Larmor mode. This mode will be characterized by its long lifetime, and we will discuss ways in which it can be experimentally observed.

\subsection{Results}

\subsubsection{DEG with spin-orbit coupling in a magnetic field: the spin helix}

We consider the electronic ground state of a 2DEG in an $n$-doped zincblende quantum well in the presence of an in-plane magnetic field and Rashba and Dresselhaus SOC. Since the magnetic field is parallel to the 2DEG, it only acts on the spin and there 


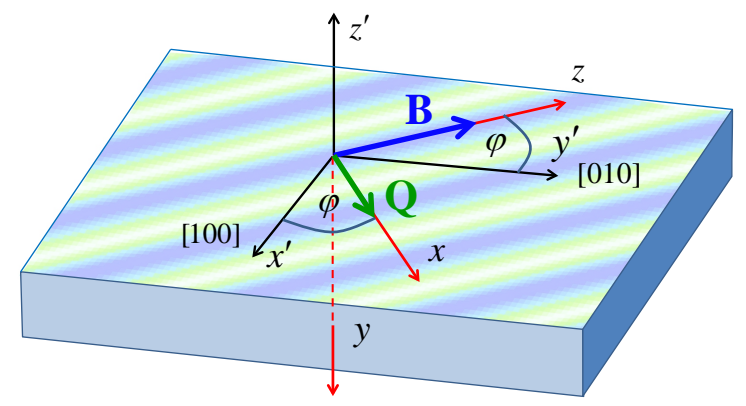

Figure 4.1: Reference frames $\mathcal{R}^{\prime}$ (black) and $\mathcal{R}$ (red) for the electronic states in a 2DEG with SOC and in-plane magnetic field $\mathbf{B}$. The striped pattern along the $\varphi=45^{\circ}$ direction indicates the persistent spin helix state of the 2DEG, with wave vector $\mathbf{Q}$, which forms in the absence of $\mathbf{B}$ if the Rashba and Dresselhaus coupling strengths are equal.

is no Landau level quantization (as long as the magnetic length $l_{B}=\sqrt{\hbar /|e B|}$ is not significantly smaller than the well width). We will use the effective-mass approximation and work in units where $\hbar=e^{*}=m^{*}=1$, where $e^{*}$ and $m^{*}$ are the effective charge and mass, respectively (This is a special case of what we did in chapter 3 , but we include the derivation here to keep chapter 4 self-contained).

Figure 4.1 defines two reference frames. The primed frame $\mathcal{R}^{\prime}$ is fixed with respect to the quantum well: the $x^{\prime}-, y^{\prime}$ - and $z^{\prime}$-axes point along the crystallographic [100], [010], and [001] directions, respectively; the 2DEG is in the $x^{\prime}-y^{\prime}$ plane. The Rashba and Dresselhaus SOC fields will be introduced in $\mathcal{R}^{\prime}$, but it will be convenient for the discussion of spin waves to work in a coordinate system $\mathcal{R}$ which is oriented such that its $x$ and $z$ axes lie in the quantum well plane, and the $z$-axis points along the in-plane magnetic field B. As shown in Fig. 4.1, the $x$-axis is at an angle $\varphi$ with respect to the $x^{\prime}$-axis. 


\section{Single-particle states}

Without magnetic field or SOC, the lowest electronic conduction subband in the quantum well is spin-degenerate. For simplicity, we will treat the electronic states as purely two-dimensional; however, the main results in this chapter will not change significantly if one takes the finite well width into account. The magnetic field lifts the degeneracy and splits the lowest subband into two, which we shall denote by the index $p= \pm 1$. In the reference frame $\mathcal{R}^{\prime}$, the associated single-particle states can be written as $\Phi_{p \mathbf{k}}^{\prime}\left(\mathbf{r}^{\prime}\right)=e^{i \mathbf{k} \cdot \mathbf{r}^{\prime}} \Psi_{p \mathbf{k}}^{\prime}$, where $\mathbf{r}^{\prime}=\left(x^{\prime}, y^{\prime}\right), \mathbf{k}=\left(k_{x^{\prime}}, k_{y^{\prime}}\right)$, and $\Psi_{p \mathbf{k}}^{\prime}$ is a two-component spinor of the form

$$
\Psi_{p \mathbf{k}}^{\prime}\left(z^{\prime}\right)=\left(\begin{array}{c}
\psi_{p \mathbf{k} \uparrow}^{\prime} \\
\psi_{p \mathbf{k} \downarrow}^{\prime}
\end{array}\right)
$$

Here, "spin-up" and "spin-down" ( $\uparrow$ and $\downarrow)$ refer to the spin quantization axis $z^{\prime}$.

The states $\Psi_{p \mathbf{k}}^{\prime}$ are obtained from the following single-particle Schrödinger equation:

$$
\left(\frac{k^{2}}{2} \hat{\sigma}_{0}+h_{x^{\prime}} \hat{\sigma}_{x^{\prime}}+h_{y^{\prime}} \hat{\sigma}_{y^{\prime}}\right) \Psi_{p \mathbf{k}}^{\prime}=E_{p \mathbf{k}} \Psi_{p \mathbf{k}}^{\prime}
$$

where $\hat{\sigma}_{0, x^{\prime}, y^{\prime}, z^{\prime}}$ are the usual Pauli matrices. The off-diagonal parts in Eq. involve

$$
\begin{aligned}
& h_{x^{\prime}}=-\frac{Z+Z_{\mathrm{xc}}}{2} \sin \varphi+\alpha k_{y^{\prime}}+\beta k_{x^{\prime}} \\
& h_{y^{\prime}}=\frac{Z+Z_{\mathrm{xc}}}{2} \cos \varphi-\alpha k_{x^{\prime}}-\beta k_{y^{\prime}} .
\end{aligned}
$$

Here, $Z=g^{*} \mu_{B} B$ is the bare Zeeman energy $\left(\mu_{B}\right.$ is the Bohr magneton, and $g^{*}$ is 
the effective $g$-factor), and $\alpha$ and $\beta$ are the usual Rashba and Dresselhaus coupling parameters. The presence of Coulomb many-body effects in the interacting 2DEG gives rise to the Zeeman exchange-correlation (xc) energy $Z_{\mathrm{xc}}$, which we discuss below.

It is convenient to change the reference frame for the spin, and go over to reference system $\mathcal{R}$, whose $z$-axis is along the magnetic field direction. We introduce two inplane vectors, $\mathbf{q}_{0}$ and $\mathbf{q}_{1}$, given in $\mathcal{R}^{\prime}$ by

$$
\begin{aligned}
& \mathbf{q}_{0}=(\alpha \cos \varphi+\beta \sin \varphi) \hat{e}_{x^{\prime}}+(\alpha \sin \varphi+\beta \cos \varphi) \hat{e}_{y^{\prime}} \\
& \mathbf{q}_{1}=(\beta \cos \varphi-\alpha \sin \varphi) \hat{e}_{x^{\prime}}+(\alpha \cos \varphi-\beta \sin \varphi) \hat{e}_{y^{\prime}} .
\end{aligned}
$$

With this, Eq. (4.2) transforms into

$$
\left[\frac{k^{2}}{2} \hat{\sigma}_{0}+\left(\frac{Z+Z_{\mathrm{xc}}}{2}-\mathbf{k} \cdot \mathbf{q}_{0}\right) \hat{\sigma}_{z}+\mathbf{k} \cdot \mathbf{q}_{1} \hat{\sigma}_{x}\right] \Psi_{p \mathbf{k}}=E_{p \mathbf{k}} \Psi_{p \mathbf{k}},
$$

where the scalar products $\mathbf{k} \cdot \mathbf{q}_{0}$ and $\mathbf{k} \cdot \mathbf{q}_{1}$ remain invariant under $\mathcal{R}^{\prime} \rightarrow \mathcal{R} . \Psi_{p \mathbf{k}}$ is now a two-component spinor whose spatial coordinates and spin quantization axes are defined with respect to $\mathcal{R}$.

Let us now discuss the xc contribution. The in-plane magnetic field causes the 2DEG to become uniformly magnetized. The xc energy per particle of a homogeneous 2DEG [80], $e_{\mathrm{xc}}(n, \zeta)$, can be written as a functional of the density $n$ and the spin polarization $\zeta$, where $n=n_{\uparrow}+n_{\downarrow}$ and $\zeta=\left(n_{\uparrow}-n_{\downarrow}\right) / n$ ( $\uparrow$ and $\downarrow$ are now defined with respect to $\hat{e}_{z}$ ). The Zeeman xc energy is then given by

$$
Z_{\mathrm{xc}}=2 \frac{\partial e_{\mathrm{xc}}}{\partial \zeta}
$$


The renormalized Zeeman energy [81] can now be defined as $Z^{*}=Z+Z_{\mathrm{xc}}$.

The general solution of Eq. (4.7) has been considered in chapter 3; instead, we concentrate here on the special case $\alpha=\beta$ and $\varphi=\pi / 4$ or $5 \pi / 4$. Under these circumstances, $\mathbf{q}_{1}=0$ and Eq. (4.7) simplifies considerably:

$$
\left[\frac{k^{2}}{2} \hat{\sigma}_{0}+\frac{Z^{*}-\mathbf{k} \cdot \mathbf{Q}}{2} \hat{\sigma}_{z}\right] \Psi_{p \mathbf{k}}=E_{p \mathbf{k}} \Psi_{p \mathbf{k}}
$$

where

$$
\mathrm{Q}= \pm 4 \alpha \hat{e}_{x}, \quad \varphi=\left\{\begin{array}{l}
\pi / 4 \\
5 \pi / 4 .
\end{array}\right.
$$

To keep the discussion a bit simpler, we will limit ourselves to the case $\varphi=\pi / 4$ in the following, so $\mathbf{Q}=4 \alpha \hat{e}_{x}$ (the $\varphi=5 \pi / 4$ case is essentially the same, just in the opposite direction).

The solution of Eq. (4.9) is straightforward. We obtain

$$
\Psi_{+, \mathbf{k}}=\left(\begin{array}{c}
1 \\
0
\end{array}\right), \quad \Psi_{-, \mathbf{k}}=\left(\begin{array}{c}
0 \\
1
\end{array}\right)
$$

where

$$
E_{ \pm, \mathbf{k}}=\frac{k^{2}}{2} \pm \frac{Z^{*}-\mathbf{k} \cdot \mathbf{Q}}{2}
$$

The single-particle energies (4.12) have the important property

$$
E_{+, \mathbf{k}+\mathbf{Q}}=E_{-, \mathbf{k}}+Z^{*},
$$

which is illustrated in Fig. 2.2b, using the parameters $Z^{*}=0.0381$ and $\alpha=0.05$. This 

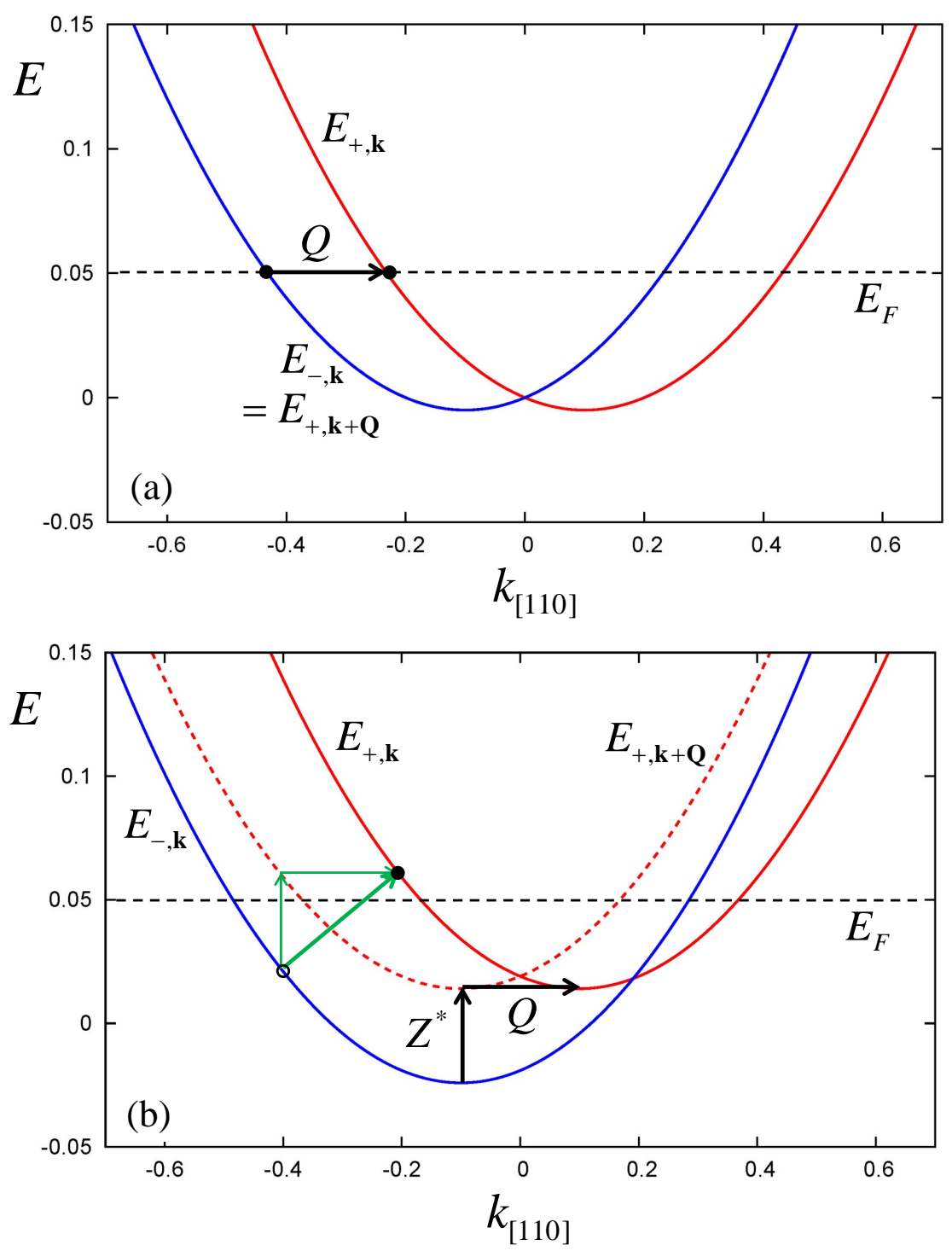

Figure 4.2: Single-particle energies $E_{-, \mathbf{k}}$ and $E_{+, \mathbf{k}}$ for $\alpha=0.05$ and $\mathbf{k}$ along the [110] direction, see Eq. (4.13). (a) No magnetic field $\left(Z^{*}=0\right)$. Linear combinations of $E_{-, \mathbf{k}}$ and $E_{+, \mathbf{k}+\mathbf{Q}}$ states have spin helix texture, but these cancel out if summed over all occupied states below $E_{F}$. A persistent spin helix appears if a quasiparticle is injected at the Fermi surface, as shown. (b) Finite magnetic field $\left(Z^{*}=0.0381\right)$. Single-particle excitations across the Fermi energy with momentum transfer Q (green arrow) give rise to propagating spin helices. 
large value of $\alpha$ (typical experimental values of $\alpha$ are about an order of magnitude smaller) was chosen for clarity of presentation.

\section{$\mathbf{B}=0:$ spin-helical single-particle eigenstates}

In the absence of external magnetic fields, i.e., for $Z^{*}=0$ (see Fig. 2.2a), the degeneracy of the two energy branches $E_{+, \mathbf{k}+\mathbf{Q}}$ and $E_{-, \mathbf{k}}$ gives rise to a persistent spin-helix state of the 2DEG, as illustrated by the stripe-like pattern in Fig. 4.1. This is easy to see: Due to the degeneracy, linear combinations of the eigenstates $\Psi_{-, \mathbf{k}}$ and $\Psi_{+, \mathbf{k}+\mathbf{Q}}$ are also solutions of Eq. (4.9). The single-particle wave functions for wave vector $\mathbf{k}$ can therefore be written as

$$
\Phi_{\mathbf{k}}^{ \pm}(\mathbf{r})=a\left(\begin{array}{c}
1 \\
0
\end{array}\right) e^{i(\mathbf{k}+\mathbf{Q}) \cdot \mathbf{r}} \pm b\left(\begin{array}{c}
0 \\
1
\end{array}\right) e^{i \mathbf{k} \cdot \mathbf{r}}
$$

where $|a|^{2}+|b|^{2}=1$. From the associated spin-density matrix it is straightforward to determine the magnetization in the $\mathcal{R}$ frame. We obtain $m_{z}=|a|^{2}-|b|^{2}$, and since the macroscopic magnetization must vanish (i.e., $m_{z}=0$ ), this implies $|a|=|b|$. Writing $a, b=e^{i \phi_{a, b}} / \sqrt{2}$ and defining $\delta_{a b}=\phi_{a}-\phi_{b}$, we get

$$
m_{x}= \pm \cos \left(\mathbf{Q} \cdot \mathbf{r}+\delta_{a b}\right), \quad m_{y}=\mp \sin \left(\mathbf{Q} \cdot \mathbf{r}+\delta_{a b}\right)
$$

Accordingly, the stripes in Fig. 4.1 indicate a periodic rotation of the electronic spin in and out of the plane, with a wave vector $\mathbf{Q}=4 \alpha \hat{e}_{x}$ oriented at a $45^{\circ}$ angle with respect to the $x^{\prime}$-axis (i.e., the [110] direction). This is the spin helix pattern $[107,114]$. 
The magnetizations $m_{x}$ and $m_{y}$ associated with the states $\Phi_{\mathbf{k}}^{+}$and $\Phi_{\mathbf{k}}^{-}$cancel out; therefore, adding up all occupied spin-helix states below the Fermi energy $E_{F}$ gives zero. This means that the ground state of the $N$-electron system has no spin texture. The persistent spin helix pattern can be observed if additional quasiparticles are injected at the Fermi level, as shown in Fig. 4.2a. Such states will have a very long lifetime [107, 109, 110].

\section{$\mathbf{B} \neq 0$ : spin-helical single-particle excitations}

For a finite magnetic field $\left(Z^{*}>0\right)$, the degeneracy of the two energy branches $E_{+, \mathbf{k}+\mathbf{Q}}$ and $E_{-, \mathbf{k}}$ is lifted, as shown in Fig. 4.2b. As a consequence, the spin-helix pattern (4.15) is not a property of the ground state anymore: instead, the spin helix becomes a nonequilibrium feature.

To see this, consider a single-particle excitation across the Fermi surface, with wave vector transfer $\mathbf{Q}$, as illustrated by the green arrow in Fig. 4.2b. First-order perturbation theory tells us that the time-dependent wave function has the form

$$
\Phi_{\mathbf{k} \rightarrow \mathbf{k}+\mathbf{Q}}(\mathbf{r}, t)=\gamma\left(\begin{array}{c}
1 \\
0
\end{array}\right) e^{i(\mathbf{k}+\mathbf{Q}) \cdot \mathbf{r}} e^{-i\left(E_{-, \mathbf{k}}+Z^{*}\right) t}+\left(\begin{array}{c}
0 \\
1
\end{array}\right) e^{i \mathbf{k} \cdot \mathbf{r}} e^{-i E_{-, \mathbf{k}} t}
$$

where $|\gamma| \ll 1$ and we made use of Eq. (4.13). In the $\mathcal{R}$ frame, the $x$ and $y$ components of the associated magnetization are given by

$$
\begin{aligned}
& m_{x}(t)=2|\gamma| \cos \left(\mathbf{Q} \cdot \mathbf{r}-Z^{*} t+\phi_{\gamma}\right) \\
& m_{y}(t)=-2|\gamma| \sin \left(\mathbf{Q} \cdot \mathbf{r}-Z^{*} t+\phi_{\gamma}\right)
\end{aligned}
$$


where $\phi_{\gamma}$ is the phase of $\gamma$. This defines a forward propagating spin helix (i.e., a spin-flip wave), with amplitude $2|\gamma|$ and group velocity $Z^{*} \mathbf{Q} / Q^{2}$. Single-particle excitations of this type, which are long-lived due to the property (4.13), were optically probed by Walser et al [110].

So far, our discussion of the excited states has ignored the effects of Coulomb interactions. In the following Section, we will consider a very special case of a collective mode, which we call the spin-helix Larmor mode, which can be viewed as a coherent superposition of left- and right-propagating single-particle spin helices considered above. As we will see, this gives rise to a collective, standing precessional wave with wave vector $\mathbf{Q}$ which is undamped. Propagating collective spin-flip waves and their wave vector dispersions will be considered in Methods.

\subsubsection{Spin-helix Larmor mode}

We consider a 2DEG in the presence of a uniform magnetic field $\mathbf{B}=B \hat{e}_{z}$, in the reference frame $\mathcal{R}$ of Fig. 4.1. The many-body Hamiltonian without SOC is

$$
\hat{H}_{0}=\sum_{i}\left(\frac{\hat{\mathbf{p}}_{i}^{2}}{2}+\frac{1}{2} Z \hat{\sigma}_{z, i}\right)+\frac{1}{2} \sum_{i j} \frac{1}{\left|\mathbf{r}_{i}-\mathbf{r}_{j}\right|}
$$

The SOC Hamiltonian for the spin-helix case is given by [see Eq. (4.9)]

$$
\hat{H}_{\mathrm{SOC}}=-\frac{1}{2} \sum_{i} \mathbf{Q} \cdot \hat{\mathbf{p}}_{i} \hat{\sigma}_{z, i} .
$$

The total Hamiltonian of the system is $\hat{H}=\hat{H}_{0}+\hat{H}_{\text {SOC }}$. We now show that the SOC contribution can be transformed away. We consider the spin-wave operator 
$[77,78,74]$

$$
\hat{S}_{+, \mathbf{q}}=\frac{1}{2} \sum_{i} \hat{\sigma}_{+, i} e^{i \mathbf{q} \cdot \mathbf{r}_{i}}
$$

$\left(\hat{\sigma}_{+}=\hat{\sigma}_{x}+i \hat{\sigma}_{y}\right)$, whose Heisenberg equation of motion is

$$
i \frac{d}{d t} \hat{S}_{+, \mathbf{q}}=\left[\hat{S}_{+, \mathbf{q}}, \hat{H}\right]=-\omega_{\mathrm{sw}}(\mathbf{q}) \hat{S}_{+, \mathbf{q}}
$$

Here, $\omega_{\text {sw }}(\mathbf{q})$ is the spin-wave dispersion, which in general has a real part and an imaginary part (related to the inverse of the lifetime). We now introduce the $\mathrm{SU}(2)$ unitary transformation $\hat{U}=\exp \left[-i \sum_{i} \mathbf{Q} \cdot \mathbf{r}_{i} \hat{\sigma}_{z, i} / 2\right]$, which leads to

$$
\hat{U} \hat{S}_{+, \mathbf{q}} \hat{U}^{\dagger}=\hat{S}_{+, \mathbf{q}-\mathbf{Q}}
$$

$\hat{U}$ causes a boost of the wave vector argument of the spin-wave operator, $\mathbf{q} \rightarrow \mathbf{q}-\mathbf{Q}$, which leaves the Coulomb and the Zeeman parts of $\hat{H}_{0}$ unchanged, and transforms the momentum operator of the $i$ th electron into $\hat{U} \hat{\mathbf{p}}_{i} \hat{U}^{\dagger}=\hat{\mathbf{p}}_{i}+\mathbf{Q} \hat{\sigma}_{z, i} / 2$. Thus, we obtain

$$
\hat{U} \hat{H} \hat{U}^{\dagger}=\hat{H}_{0}-\frac{Q^{2}}{8} \hat{\sigma}_{0}
$$

Since $Q^{2} \hat{\sigma}_{0} / 8$ commutes with $\hat{S}_{+, \mathbf{q}-\mathbf{Q}}$, the transformed equation of motion (4.22) becomes

$$
\left[\hat{S}_{+, \mathbf{q}-\mathbf{Q}}, \hat{H}_{0}\right]=-\omega_{\mathrm{sw}}(\mathbf{q}) \hat{S}_{+, \mathbf{q}-\mathbf{Q}} .
$$

Therefore, the spin waves of the system with $\alpha=\beta$ are those of the system without SOC (governed only by $\hat{H}_{0}$ ), but where the wave vector is shifted:

$$
\omega_{\mathrm{sw}}(\mathbf{q})=\omega_{\mathrm{sw}, 0}(\mathbf{q}-\mathbf{Q}) .
$$


Let us now consider the important special case $\mathbf{q}=\mathbf{Q}$. It is known [58] that, in the absence of SOC, the spin-polarized electron system carries out a collective precessional motion with $\omega_{\mathrm{sw}, 0}=\omega_{L}$. The Larmor frequency $\omega_{L}$ is equal to the bare Zeeman energy, $\omega_{L}=Z$. The Larmor mode has infinite lifetime (zero line width), because it can be represented as a superposition of two exact eigenstates of the system Hamiltonian $\hat{H}_{0}$ : the many-body ground state $|0\rangle_{0}$ (the subscript 0 indicates absence of SOC), with ground-state energy $E_{0}$, and the many-body eigenstate $\hat{S}_{+, 0}|0\rangle_{0}$, with energy $E_{0}+Z$.

Coming back to the case with SOC with the full Hamiltonian $\hat{H}$, we can now formulate the spin-helix Larmor theorem. If the spin wave has wave vector $\mathbf{Q}$, commensurate with the spin-helix texture, all Coulomb many-body contributions drop out and the frequency is given by the bare Zeeman energy:

$$
\omega_{\mathrm{sw}}(\mathbf{Q})=\omega_{\mathrm{sw}, 0}(0)=Z
$$

which follows directly from Eq. (4.26). The spin wave then has vanishing group velocity, $\left.\nabla_{\mathbf{q}} \omega_{\mathrm{sw}}(\mathbf{q})\right|_{\mathbf{q}=\mathbf{Q}}=0$, which means that it is a standing wave. All electronic spins precess about their local orientation, given by the spin helix configuration, with the Larmor frequency $\omega_{L}=Z$. The spin-helix Larmor mode is a superposition of two many-body eigenstates of $\hat{H}$ : the ground state $|0\rangle$ and the state $\hat{S}_{+, \mathbf{Q}}|0\rangle$.

An important feature of the Larmor's mode is that it does not carry any spin current in the plane. This is obvious in absence of SOC, where the exact equation of motion for $\hat{S}_{+, \mathbf{q}}$ reads $[79]$ :

$$
i \frac{d}{d t} \hat{S}_{+, \mathbf{q}}=\left[\hat{S}_{+, \mathbf{q}}, \hat{H}_{0}\right]=-\omega_{L} \hat{S}_{+, \mathbf{q}}-\mathbf{q} \cdot \hat{\mathbf{J}}_{\mathbf{q}}^{+}
$$


Here, $\hat{\mathbf{J}}_{\mathbf{q}}^{+}$is the transverse spin-current operator at wave vector $\mathbf{q}$. The second term on the right-hand side arises from the commutator of $\hat{S}_{+, \mathbf{q}}$ with the kinetic part of $\hat{H}_{0}$. Hence, for the Larmor's mode which occurs at $\mathbf{q}=0$, no current is induced by the homogenous precession. In the presence of SOC, the Larmor's mode occurs at $\mathbf{q}=\mathbf{Q}$ and is not a homogenous mode anymore; however, one still has the property that no spin-current is driven by the precession. Indeed, the exact equation of motion for $\hat{S}_{+, \mathbf{q}}$ in the presence of SOC is given by

$$
i \frac{d}{d t} \hat{S}_{+, \mathbf{q}}=\left[\hat{S}_{+, \mathbf{q}}, \hat{H}_{0}+\hat{H}_{\mathrm{SOC}}\right]=-\omega_{L} \hat{S}_{+, \mathbf{q}}+(-\mathbf{q}+\mathbf{Q}) \cdot \hat{\mathbf{J}}_{\mathbf{q}}^{+} .
$$

Hence, when $\mathbf{q}=\mathbf{Q}$ the total current term disappears. SOC induces spin currents opposite to the spin currents induced by the motion.

We illustrate the spin-wave dispersions with and without SOC in Fig. 4.3. The left panel shows $\omega_{\mathrm{sw}, 0}(\mathbf{q})$ (which is independent of the direction of $\mathbf{q}$ ), and the right panel shows $\omega_{\text {sw }}(\mathbf{q})$ for $\mathbf{q}$ parallel to $\mathbf{Q}$, i.e., along the [110] direction. For this particular case, $\omega_{\mathrm{sw}}(\mathbf{q})$ is simply obtained by a horizontal shift by $\mathbf{Q}$ of $\omega_{\mathrm{sw}, 0}(\mathbf{q})$ (likewise for the particle-hole continua). The spin-wave dispersions plotted in Fig. 4.3 are obtained from the numerical solution of Eq. (4.39), see below. The small-wave vector expansion (4.41) is very close to the exact result.

\subsubsection{Experimental schemes}

Experimental observation of the the spin-helix Larmor mode should be possible in specially designed doped semiconductor quantum well samples where the $\alpha=\beta$ condition is met. The spin-flip waves under an in-plane magnetic field can be detected 

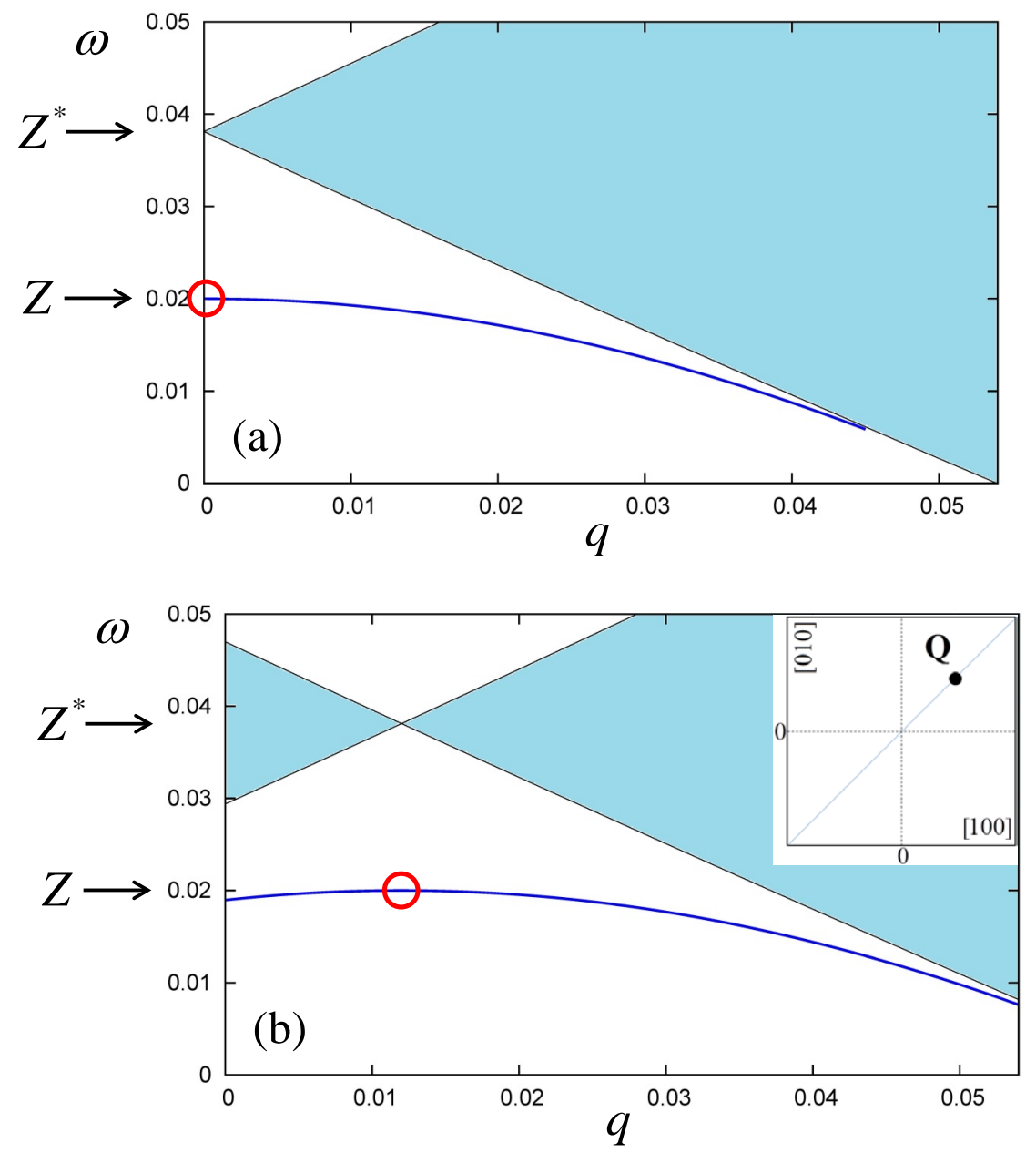

Figure 4.3: (a) Spin-wave dispersion $\omega_{\mathrm{sw}, 0}(\mathbf{q})$ (line) and single-particle spin-flip continuum (shaded area) without SOC. (b) Spin-wave dispersion $\omega_{\mathrm{sw}}(\mathbf{q})$ and single-particle spin-flip continuum, plotted along [110], for $\alpha=\beta=0.003(Q=0.012)$. The 2DEG parameters are $r_{s}=2, \zeta=-0.0762, Z=0.02$, and $Z^{*}=0.0381$ (all values are in atomic units). The inset shows the position of Larmor's mode in the wave vector plane $\left(q_{x^{\prime}}, q_{y^{\prime}}\right)$. 
using inelastic light scattering, similar to our earlier work [71, 72, 73, 74]. The Larmortype precessional mode about the virtual spin-helix state should then be recognizable by a significant narrowing of the linewidth.

We also propose a device design which would allow to excite Larmor's mode optically and probe it electronically. As shown in Fig. 4.4, the idea would be to deposit metal stripes on top of the sample, separated by a distance of $2 \pi / Q$ (for typical values of $\alpha$, of order $\sim 1 \mathrm{meV} \AA$, this corresponds to a few $\mu \mathrm{m}$ ). The stripes are aligned parallel to the applied magnetic field, perpendicular to the [010] direction, which is commensurate with the standing-wave spin-helix Larmor mode. The metal stripes represent a grating coupler $[120,121,122]$, which acts as an antenna and transducer for short, few-cycle microwave pulses impinging on the sample. By induction, the alternating currents in the metal stripes generate concentric magnetic fields, which exert torques on the spin-polarized 2DEG underneath. If the right frequency, $\omega_{L}$, is

chosen, this will trigger a helical standing spin wave which will persist after the end of the pulse. Detection of the spin-helix Larmor mode, and measurement of its lifetime, should then be possible via the currents induced in the metal stripes from the stray magnetic fields associated with the spin wave.

\subsection{Discussion}

In this chapter, we have considered the spin dynamics in a 2DEG in the presence of SOC, under the very special condition where the Rashba and Dresselhaus coupling strengths are equal $(\alpha=\beta)$ and where an in-plane magnetic field is applied perpendicular to the [110] direction. Without this magnetic field, the system sustains persistent 


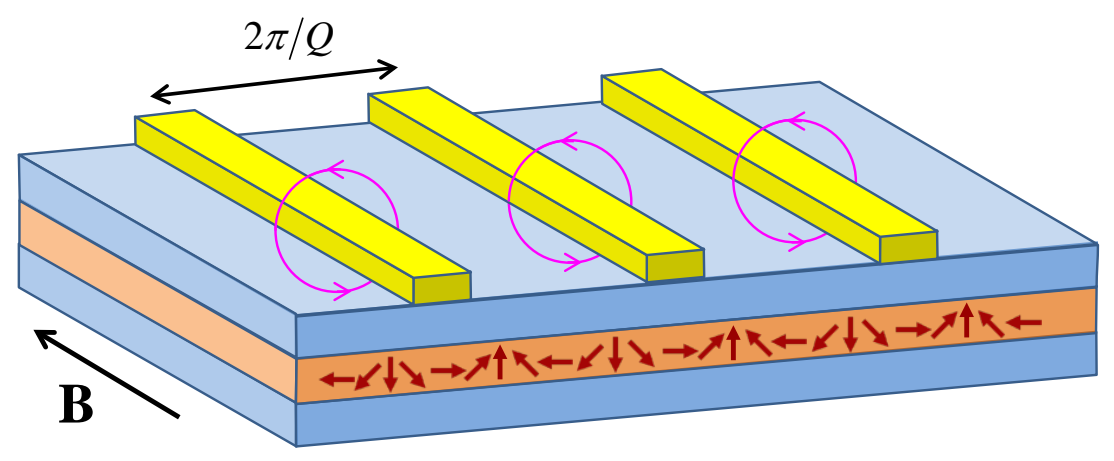

Figure 4.4: Proposed experimental design of the optical excitation and subsequent detection of the spin-helix Larmor mode using a grating coupler (see text for details).

spin-helix states which have been widely studied in the literature. The magnetic field lifts the degeneracy that leads to the persistent spin-helix states; it instead leads to single-particle excitations that have the form of propagating spin helices.

The presence of Coulomb interactions causes these single-particle excitations to combine and form collective spin waves, which are robust against any decoherence caused by SOC. We have found that for the 2DEG with $\alpha=\beta$ the spin-wave dispersion is the same as for the system without SOC, apart for a rigid wave vector shift by $\mathbf{Q}$ (the spin-helix wave vector). The case of $\mathbf{q}=\mathbf{Q}$ thus produces the special scenario which we have termed the spin-helix Larmor mode, where all many-body effects vanish and the precession frequency is given by the bare Zeeman energy (divided by $\hbar)$. This is a new and exact result for electronic many-body systems, which opens up new ways of manipulating and driving electronic spins by optical means. 


\subsection{Methods}

We now discuss the spin-wave dispersions in the quantum well system considered above. We have seen that in the case $\alpha=\beta$ the single-particle states (4.11) are pure up and down spinors. Therefore the longitudinal and transverse spin response channels are decoupled, and the noninteracting transverse spin response function is a diagonal $2 \times 2$ matrix in frame $\mathcal{R}[2,78]$ :

$$
\underline{\chi}_{T}(\mathbf{q}, \omega)=\left(\begin{array}{cc}
\chi_{\uparrow \downarrow, \uparrow \downarrow}(\mathbf{q}, \omega) & 0 \\
0 & \chi_{\downarrow \uparrow, \downarrow \uparrow}(\mathbf{q}, \omega)
\end{array}\right) .
$$

The general form of the individual elements of the noninteracting spin-density-matrix response function for a $2 \mathrm{DEG}$ is [29]

$$
\begin{aligned}
\chi_{\sigma \sigma^{\prime}, \tau \tau^{\prime}}(\mathbf{q}, \omega)= & -\sum_{p p^{\prime}}^{ \pm 1} \int \frac{d^{2} k}{(2 \pi)^{2}} \frac{f\left(E_{p \mathbf{k}}\right)}{\omega-E_{p \mathbf{k}}+E_{p^{\prime} \mathbf{k}-\mathbf{q}}+i \eta} \\
& \times\left\{\left[\delta_{p,+1} \delta_{\sigma \uparrow}+\delta_{p,-1} \delta_{\sigma \downarrow}\right]\left[\delta_{p^{\prime},+1} \delta_{\sigma^{\prime} \uparrow}+\delta_{p^{\prime},-1} \delta_{\sigma^{\prime} \downarrow}\right] \delta_{\sigma \tau} \delta_{\sigma^{\prime} \tau^{\prime}}\right\} \\
+ & \sum_{p p^{\prime}}^{ \pm 1} \int \frac{d^{2} k}{(2 \pi)^{2}} \frac{f\left(E_{p \mathbf{k}}\right)}{\omega+E_{p \mathbf{k}}-E_{p^{\prime} \mathbf{k}+\mathbf{q}}+i \eta} \\
& \times\left\{\left[\delta_{p^{\prime},+1} \delta_{\sigma \uparrow}+\delta_{p^{\prime},-1} \delta_{\sigma \downarrow}\right]\left[\delta_{p,+1} \delta_{\sigma^{\prime} \uparrow}+\delta_{p,-1} \delta_{\sigma^{\prime} \downarrow}\right] \delta_{\sigma \tau} \delta_{\sigma^{\prime} \tau^{\prime}}\right\}
\end{aligned}
$$

where $\sigma, \sigma^{\prime}, \tau, \tau^{\prime}$ are spin indices ( $\uparrow$ or $\downarrow$ ), and $\eta$ is a positive infinitesimal (since we will be considering spin waves outside the particle-hole continuum, we can drop $\eta$ ). The Fermi function is given by $f\left(E_{p \mathbf{k}}\right)=\theta\left(E_{F}-E_{p \mathbf{k}}\right)$, where $E_{F}$ is the Fermi energy of a paramagnetic 2DEG in the presence of SOC. It can be shown that $E_{F}=E_{F}^{0}-\alpha^{2}-\beta^{2}$, where $E_{F}^{0}=\pi n$ is the Fermi energy of a $2 \mathrm{DEG}$ without SOC, and with $2 \mathrm{D}$ electronic density $n$. 
We recast Eq. (4.12) as $E_{ \pm, \mathbf{k}}= \pm Z^{*} / 2+\frac{1}{2}|\mathbf{k} \mp \mathbf{Q} / 2|^{2}-2 \alpha^{2}$. With a change of the integration variable, $\mathbf{k} \rightarrow \mathbf{k} \pm \mathbf{Q} / 2$, the noninteracting spin-flip response functions become

$$
\begin{aligned}
\chi_{\uparrow \downarrow, \uparrow \downarrow}(\mathbf{q}, \omega) & =-\int \frac{d^{2} k}{(2 \pi)^{2}} \frac{f_{0}\left(\frac{k^{2}}{2}+\frac{Z^{*}}{2}\right)}{\omega-Z^{*}-\mathbf{k} \cdot(\mathbf{q}-\mathbf{Q})+|\mathbf{q}-\mathbf{Q}|^{2} / 2} \\
& +\int \frac{d^{2} k}{(2 \pi)^{2}} \frac{f_{0}\left(\frac{k^{2}}{2}-\frac{Z^{*}}{2}\right)}{\omega-Z^{*}-\mathbf{k} \cdot(\mathbf{q}-\mathbf{Q})-|\mathbf{q}-\mathbf{Q}|^{2} / 2} \\
\chi_{\downarrow \uparrow, \downarrow \uparrow}(\mathbf{q}, \omega) & =\int \frac{d^{2} k}{(2 \pi)^{2}} \frac{f_{0}\left(\frac{k^{2}}{2}+\frac{Z^{*}}{2}\right)}{\omega+Z^{*}-\mathbf{k} \cdot(\mathbf{q}+\mathbf{Q})-|\mathbf{q}+\mathbf{Q}|^{2} / 2} \\
& -\int \frac{d^{2} k}{(2 \pi)^{2}} \frac{f_{0}\left(\frac{k^{2}}{2}-\frac{Z^{*}}{2}\right)}{\omega+Z^{*}-\mathbf{k} \cdot(\mathbf{q}+\mathbf{Q})+|\mathbf{q}+\mathbf{Q}|^{2} / 2},
\end{aligned}
$$

where the Fermi function $f_{0}$ indicates that $E_{F}$ has been replaced by $E_{F}^{0}$.

Equations (4.32) and (4.33) tell us that the spin-flip response functions of the system with SOC can be expressed in terms of the corresponding functions without SOC (denoted by the superscript 0):

$$
\begin{aligned}
& \chi_{\uparrow \downarrow, \uparrow \downarrow}(\mathbf{q}, \omega)=\chi_{\uparrow \downarrow, \uparrow \downarrow}^{0}(\mathbf{q}-\mathbf{Q}, \omega) \\
& \chi_{\downarrow \uparrow, \downarrow \uparrow}(\mathbf{q}, \omega)=\chi_{\downarrow \uparrow, \downarrow \uparrow}^{0}(\mathbf{q}+\mathbf{Q}, \omega) .
\end{aligned}
$$

This simple result only holds for the special case $\alpha=\beta$. In TDDFT, the spinflip linear-response xc kernel of the 2DEG is given in the adiabatic local-density approximation (ALDA) by [29]

$$
f_{\uparrow \downarrow, \uparrow \downarrow}^{\mathrm{xc}}=f_{\downarrow \uparrow, \downarrow \uparrow}^{\mathrm{xc}} \equiv K_{\mathrm{xc}}=\frac{2}{n \zeta} \frac{\partial e_{\mathrm{xc}}}{\partial \zeta} .
$$




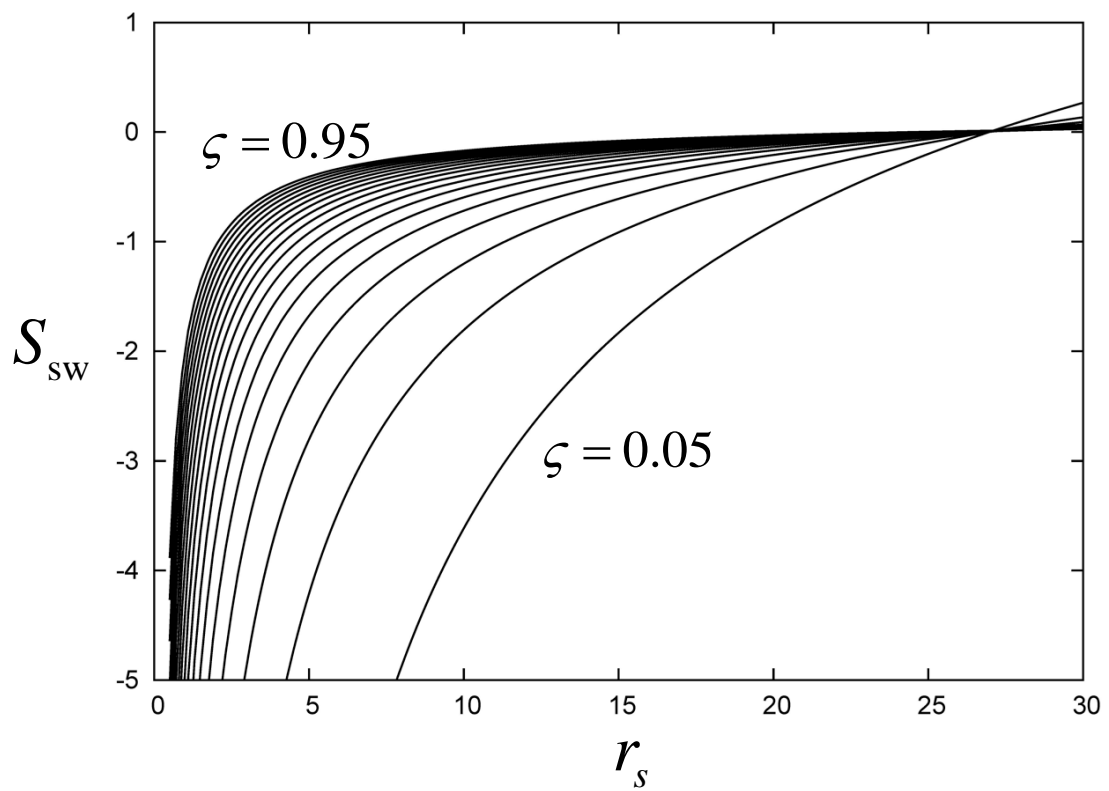

Figure 4.5: Spin-wave stiffness of the 2DEG, obtained with the ALDA, for various values of the spin polarization $\zeta$ between 0.05 and 0.95 .

The spin-flip wave dispersion now follows from solving

$$
\left[K_{\mathrm{xc}} \chi_{\uparrow \downarrow, \uparrow \downarrow}^{0}\left(\mathbf{q}-\mathbf{Q}, \omega_{\mathrm{sw}}\right)-1\right]\left[K_{\mathrm{xc}} \chi_{\downarrow \uparrow, \downarrow \uparrow}^{0}\left(\mathbf{q}+\mathbf{Q}, \omega_{\mathrm{sw}}\right)-1\right]=0
$$

Upon closer inspection of Eqs. (4.32) and (4.33), we find

$$
\chi_{\downarrow \uparrow, \downarrow \uparrow}^{0}\left(\mathbf{q}+\mathbf{Q}, \omega_{\mathrm{sw}}\right)=\chi_{\uparrow \downarrow, \uparrow \downarrow}^{0}\left(\mathbf{q}-\mathbf{Q},-\omega_{\mathrm{sw}}\right) .
$$

We are only interested in positive frequencies, so the spin-flip wave dispersion is obtained from Eq. (4.37) as the implicit solution $\omega_{\mathrm{sw}}(\mathbf{q})$ of

$$
\chi_{\uparrow \downarrow, \uparrow \downarrow}^{0}\left(\mathbf{q}-\mathbf{Q}, \omega_{\mathrm{sw}}\right)=K_{\mathrm{xc}}^{-1} .
$$


The spin-wave dispersion can be analytically determined for small wave vectors. To second order, we find

$$
\omega_{\mathrm{sw}}(\mathbf{q})=Z^{*}\left(1+\frac{K_{\mathrm{xc}}}{2 \pi}\right)+\frac{E_{F}^{0}|\mathbf{q}-\mathbf{Q}|^{2}}{Z^{*}}\left(1+\frac{2 \pi}{K_{\mathrm{xc}}}\right) .
$$

This can be rewritten as

$$
\omega_{\mathrm{sw}}(\mathbf{q})=Z+\frac{1}{2} S_{\mathrm{sw}}|\mathbf{q}-\mathbf{Q}|^{2},
$$

where the spin-wave stiffness of the 2DEG is

$$
S_{\mathrm{sw}}=\frac{2 E_{F}^{0}}{Z^{*}}\left(1+\frac{2 \pi}{K_{\mathrm{xc}}}\right) .
$$

Since $K_{\mathrm{xc}}=Z_{\mathrm{xc}} /(n \zeta)$ and $\zeta=-Z^{*} /(2 \pi n)$, this can also be written as

$$
S_{\mathrm{sw}}=-\frac{2 E_{F}^{0}}{Z^{*}}\left(\frac{Z}{Z^{*}-Z}\right) .
$$

We plot the ALDA spin-wave stiffness for various values of the spin polarization $\zeta$ and as a function of the 2D Wigner-Seitz radius $r_{s}$ in Fig. 4.5. The stiffness has negative values for all practically relevant values of $r_{s}$, and crosses over to $S_{\mathrm{sw}}>0$ for very low densities (at $r_{s}=26.96$ for $\zeta=0.05$ and at $r_{s}=24.49$ for $\zeta=0.95$ ). 


\section{Chapter 5}

\section{Summary}

In this dissertation, we have presented new theoretical developments and numerical calculations in the area of semiconductor and many-body physics. Specifically, we have studied various properties of quasi-two-dimensional electron gases confined in semiconductor quantum wells. The main methodology in this work was timedependent density-functional theory (TDDFT).

We first demonstrated that the TDDFT description of electron dynamics in lowdimensional systems crucially depends on the proper choice of exchange-correlation

functional. Approximations that are based on the 2D or 3D homogeneous electron gas as reference system (semilocal functionals such as ALDA or GGA) will fail in situations that cross over between 2D and 3D. Instead, functionals that do not rely on a reference system, such as the orbital-based PGG functional (an approximation for exact exchange), perform much better during dimensional crossover.

We then studied 2DEGs in $n$-doped quantum wells under the presence of in-plane magnetic fields and Rashba and Dresselhaus spin-orbit coupling. We took the SOC 
effects into the account, up to the second order, and found that Larmor's theorem no longer holds, and there is a small correction to the spin-wave stiffness in the spin-wave dispersion. A detailed comparison with experimental results from inelastic light scattering confirmed that our linear-response TDDFT approach, based on the effective-mass approximation, is very accurate indeed. The systems under study were sufficiently far away from the 3D-2D crossover point where semilocal exchangecorrelation functionals collapse, so it was safe to use the ALDA here. In spite of that, we discovered some quantitative shortcomings of the ALDA in the calculation of the effective Zeeman splitting.

Lastly we considered the very special condition where the Rashba and Dresselhaus coupling coupling strengths are equal and the in-plane magnetic field is applied perpendicular to the [110] crystallographic direction (assuming a zincblende semiconductor system). This situation gives rise to the so-called persistent spin-helix states. In our work, we shed new light on the spin helix by including Coulomb interactions. We found that apart from a shift by the spin-helix wave vector, the spin-wave dispersion is identical to the system without SOC. This result comes out in TDDFT, and we could also prove it for general many-body Hamiltonians. Thus, we discovered a new exact result of many-body physics, which we call the spin-helix Larmor mode. In the hope that this will stimulate some experimental work, we proposed a design for an experiment to observe spin-helix Larmor modes.

The insights gained in this work open up many new research opportunities. The interplay of spin-orbit and Coulomb many-body effects continues to be of great interest. Interesting studies can be carried out to explore the impact of spin-orbit interactions on collective spin-density and spin-flip excitation in low-dimensional systems. 
On a fundamental level, collective spin-density excitations in spin-polarized 2DEGs are an ideal testing ground for new exchange-correlation functionals in TDDFT. As we have pointed out, standard approaches such as ALDA and GGA suffer from the dimensional cross-over problem, and, in addition, are not very quantitatively accurate. New functionals for noncollinear magnetism are therefore needed. The PGG functional is promising, but does not contain any correlation effects. One possibility to include the latter is via the STLS formalism, which goes back to the work of Singwi, Tosi, Land and Sjolander [123], who developed an exchange-correlation kernel for the homogeneous electron gas which was highly successful, performing much better than the RPA or other simpler methods. Generalizing the STLS methods for systems with noncollinear spin (such as the spin-orbit coupled 2DEGs considered here) would be a promising follow-up to the work done in this thesis.

On a more practical level, the methodologies developed in this thesis are very well suited to be applied to other systems besides electron gases in doped semiconductor quantum wells. There are new classes of low-dimensional systems with fascinating electronic properties, such as graphene or beyond-graphene materials such as transition metal dichalcogenides, or systems with topological properties. In these systems, Coulomb many-body effects are often only considered to be playing a minor role. However, as we have seen, the interplay between Coulomb interactions and spin-orbit coupling can have very intriguing properties. The framework developed in this thesis will make such studies possible. 


\section{Appendix A}

\section{The PBE exchange kernel}

\section{A.1 PBE exchange energy}

The PBE exchange energy functional is defined as [38]

$$
E_{\mathrm{x}}^{\mathrm{PBE}}[n]=\int d^{3} r^{\prime} e_{\mathrm{x}}^{h}(n)\left[1+\kappa-\frac{\kappa}{1+\mu s^{2} / \kappa}\right]
$$

Here, the exchange energy density of a homogeneous 3D electron liquid of density $n$ is

$$
e_{\mathrm{x}}^{h}(n)=-\frac{3 c}{4} n^{4 / 3}, \quad c=\left(\frac{3}{\pi}\right)^{1 / 3} .
$$

In Eq. (A.1), $\kappa=0.804$ and $\mu=0.21951$ are parameters given in atomic units. The quantity $s$ is defined as $s=|\nabla n| / 2 n k_{F}^{3 \mathrm{D}}$, where $k_{F}^{3 \mathrm{D}}=\left(3 \pi^{2} n\right)^{1 / 3}$ is the Fermi wavevector. Thus,

$$
s=\frac{|\nabla n|}{2\left(3 \pi^{2}\right)^{1 / 3} n^{4 / 3}} .
$$


Putting this into Eq. (A.1), we obtain

$$
E_{\mathrm{x}}^{\mathrm{PBE}}[n]=\int d^{3} r^{\prime} e_{\mathrm{x}}^{h}(n)\left[1+\kappa-\frac{\kappa}{1+\gamma|\nabla n|^{2} / n^{8 / 3}}\right],
$$

where $\gamma=(\mu / 4 \kappa)\left(3 \pi^{2}\right)^{-2 / 3}=0.007132$ a.u. For what follows, it is convenient to introduce the abbreviation

$$
g(\mathbf{r})=1+\gamma|\nabla n(\mathbf{r})|^{2} / n(\mathbf{r})^{8 / 3} .
$$

\section{A.2 PBE exchange potential}

The PBE exchange potential in its spin-unresolved form is given by

$$
\begin{aligned}
v_{\mathrm{x}}^{\mathrm{PBE}}(\mathbf{r}) & =\frac{\delta E_{\mathrm{x}}^{\mathrm{PBE}}[n]}{\delta n(\mathbf{r})} \\
& =\int d^{3} r^{\prime}\left(\frac{\delta e_{\mathrm{x}}^{h}\left(n\left(\mathbf{r}^{\prime}\right)\right)}{\delta n(\mathbf{r})}\right)\left[1+\kappa-\frac{\kappa}{g\left(\mathbf{r}^{\prime}\right)}\right] \\
& -\int d^{3} r^{\prime} e_{\mathrm{x}}^{h}\left(n\left(\mathbf{r}^{\prime}\right)\right) \frac{\delta}{\delta n(\mathbf{r})}\left(\frac{\kappa}{g\left(\mathbf{r}^{\prime}\right)}\right) .
\end{aligned}
$$

The first part is easy, with

$$
\frac{\delta e_{\mathrm{x}}^{h}\left(n\left(\mathbf{r}^{\prime}\right)\right)}{\delta n(\mathbf{r})}=-c n\left(\mathbf{r}^{\prime}\right)^{1 / 3} \delta\left(\mathbf{r}^{\prime}-\mathbf{r}\right)
$$


The second part requires more effort, involving functional derivatives of the gradient of $n$, which leads to gradients of delta functions. The final result is

$$
\begin{aligned}
v_{\mathrm{x}}^{\mathrm{PBE}}(\mathbf{r}) & =-c n(\mathbf{r})^{1 / 3}\left[1+\kappa-\frac{\kappa}{g(\mathbf{r})}\right] \\
& +\frac{3 c}{4} n(\mathbf{r})^{-4 / 3} \nabla\left[\frac{2 \kappa \gamma}{g(\mathbf{r})^{2}}\right] \cdot \nabla n(\mathbf{r}) \\
& +\frac{3 c}{4} n(\mathbf{r})^{-4 / 3} \frac{2 \kappa \gamma}{g(\mathbf{r})^{2}} \nabla^{2} n(\mathbf{r})
\end{aligned}
$$

The spin-dependent version of the PBE exchange energy functional follows from the spin-scaling relation

$$
E_{\mathrm{x}}\left[n_{\uparrow}, n_{\downarrow}\right]=\frac{1}{2} E_{\mathrm{x}}\left[2 n_{\uparrow}\right]+\frac{1}{2} E_{\mathrm{x}}\left[2 n_{\downarrow}\right] .
$$

This gives the spin-resolved exchange potential

$$
v_{\mathrm{x} \sigma}^{\mathrm{PBE}}(\mathbf{r})=v_{\mathrm{x}}^{\mathrm{PBE}}\left[2 n_{\sigma}\right](\mathbf{r}) .
$$

For a system whose density is not spin polarized we have $n_{\uparrow}=n_{\downarrow}=n / 2$. In this case, all potentials are the same, i.e., $v_{\mathrm{x} \uparrow}^{\mathrm{PBE}}(\mathbf{r})=v_{\mathrm{x} \downarrow}^{\mathrm{PBE}}(\mathbf{r})=v_{\mathrm{x}}^{\mathrm{PBE}}(\mathbf{r})$.

\section{A.3 PBE exchange kernel}

The parallel-spin exchange kernel is defined as follows:

$$
f_{\mathrm{x}, \sigma \sigma}^{\mathrm{PBE}}\left(\mathbf{r}, \mathbf{r}^{\prime}\right)=\frac{\delta v_{\mathrm{x} \sigma}^{\mathrm{PBE}}(\mathbf{r})}{\delta n_{\sigma}\left(\mathbf{r}^{\prime}\right)}
$$


(in the exchange-only case, the antiparallel-spin kernel is zero). For spin-unpolarized systems, we have

$$
f_{\mathrm{x}, \uparrow \uparrow}^{\mathrm{PBE}}\left(\mathbf{r}, \mathbf{r}^{\prime}\right)=f_{\mathrm{x}, \downarrow \downarrow}^{\mathrm{PBE}}\left(\mathbf{r}, \mathbf{r}^{\prime}\right)=2 f_{\mathrm{x}}^{\mathrm{PBE}}\left(\mathbf{r}, \mathbf{r}^{\prime}\right),
$$

where $f_{\mathrm{x}}^{\mathrm{PBE}}\left(\mathbf{r}, \mathbf{r}^{\prime}\right)=\delta v_{\mathrm{x}}^{\mathrm{PBE}}[n](\mathbf{r}) / \delta n\left(\mathbf{r}^{\prime}\right)$. After a rather lengthy calculation, one obtains

$$
\begin{aligned}
f_{\mathrm{x}}^{\mathrm{PBE}}\left(\mathbf{r}, \mathbf{r}^{\prime}\right) & =-\frac{c}{3} n(\mathbf{r})^{-2 / 3} \delta\left(\mathbf{r}-\mathbf{r}^{\prime}\right)\left[1+\kappa-\frac{\kappa}{g(\mathbf{r})}\right] \\
& -c n(\mathbf{r})^{1 / 3} \frac{\kappa \gamma}{g(\mathbf{r})^{2}} h\left(\mathbf{r}, \mathbf{r}^{\prime}\right) \\
& -c n(\mathbf{r})^{-7 / 3} \delta\left(\mathbf{r}-\mathbf{r}^{\prime}\right) \nabla\left[\frac{2 \kappa \gamma}{g(\mathbf{r})^{2}}\right] \cdot \nabla n(\mathbf{r}) \\
& -\frac{3 c}{4} n(\mathbf{r})^{-4 / 3} \nabla n(\mathbf{r}) \cdot \nabla\left(\frac{4 \kappa \gamma^{2}}{g(\mathbf{r})^{3}} h\left(\mathbf{r}, \mathbf{r}^{\prime}\right)\right) \\
& +\frac{3 c}{4} n(\mathbf{r})^{-4 / 3} \nabla\left[\frac{2 \kappa \gamma}{g(\mathbf{r})^{2}}\right] \cdot \nabla \delta\left(\mathbf{r}-\mathbf{r}^{\prime}\right) \\
& -c n(\mathbf{r})^{-7 / 3} \delta\left(\mathbf{r}-\mathbf{r}^{\prime}\right) \frac{2 \kappa \gamma}{g(\mathbf{r})^{2}} \nabla^{2} n(\mathbf{r}) \\
& +\frac{3 c}{4} n(\mathbf{r})^{-4 / 3} \frac{2 \kappa \gamma}{g(\mathbf{r})^{2}} \nabla^{2} \delta\left(\mathbf{r}-\mathbf{r}^{\prime}\right) \\
& -\frac{3 c}{4} n(\mathbf{r})^{-4 / 3} \nabla^{2} n(\mathbf{r}) \frac{4 \kappa \gamma^{2}}{g(\mathbf{r})^{3}} h\left(\mathbf{r}, \mathbf{r}^{\prime}\right)
\end{aligned}
$$

where we defined

$$
h\left(\mathbf{r}, \mathbf{r}^{\prime}\right)=\frac{2 \nabla n(\mathbf{r}) \cdot \nabla \delta\left(\mathbf{r}-\mathbf{r}^{\prime}\right)}{n(\mathbf{r})^{8 / 3}}-\frac{8|\nabla n(\mathbf{r})|^{2}}{3 n(\mathbf{r})^{11 / 3}} \delta\left(\mathbf{r}-\mathbf{r}^{\prime}\right) .
$$

To calculate excitation energies, one needs matrix elements of the exchange kernel. We here consider the case of quantum wells where everything becomes a function of $z$ and $z^{\prime}$, and we limit ourselves to intersubband excitations in the quasi-2D limit. 
Then, only the following matrix element is needed:

$$
K_{12}=\int d z \int z^{\prime} \varphi_{1}(z) \varphi_{2}(z) f_{\mathrm{x}}^{\mathrm{PBE}}\left(z, z^{\prime}\right) \varphi_{1}\left(z^{\prime}\right) \varphi_{2}\left(z^{\prime}\right) .
$$

With the explicit form (A.12) of the PBE exchange kernel, and abbreviating $\xi(z)=$ $\varphi_{1}(z) \varphi_{2}(z)$, one obtains

$$
\begin{aligned}
K_{12} & =-\frac{c}{3} \int d z \xi(z)^{2} n(z)^{-2 / 3}(1+\kappa) \\
& +\frac{c \kappa}{3} \int d z \xi(z)^{2} \frac{n(z)^{-2 / 3}}{g(z)} \\
& +2 c \kappa \gamma \int d z \xi(z) \frac{\partial}{\partial z}\left(\frac{\xi(z) n^{\prime}(z)}{g(z)^{2} n(z)^{7 / 3}}\right) \\
& +\frac{8 c}{3} \kappa \gamma \int d z \xi(z)^{2} \frac{n^{\prime}(z)^{2}}{n(z)^{10 / 3} g(z)^{2}} \\
& -2 c \kappa \gamma \int d z \xi(z)^{2} n(z)^{-7 / 3} n^{\prime}(z) \frac{\partial}{\partial z}\left(\frac{1}{g(z)^{2}}\right) \\
& -6 c \kappa \gamma^{2} \int d z \xi(z) \frac{\partial}{\partial z}\left(\frac{n^{\prime}(z) \frac{\partial}{\partial z}\left(\xi(z) n^{\prime}(z) n(z)^{-4 / 3}\right)}{g(z)^{3} n(z)^{8 / 3}}\right) \\
& -8 c \kappa \gamma^{2} \int d z \xi(z) \frac{n^{\prime}(z)^{2}}{n(z)^{11 / 3} g(z)^{3}} \frac{\partial}{\partial z}\left(\xi(z) n^{\prime}(z) n(z)^{-4 / 3}\right) \\
& -\frac{3 c}{2} \kappa \gamma \int d z \xi(z) \frac{\partial}{\partial z}\left[\xi(z) n(z)^{-4 / 3} \frac{\partial}{\partial z}\left(\frac{1}{g(z)^{2}}\right)\right] \\
& -2 c \kappa \gamma \int d z \xi(z)^{2} \frac{n(z)^{-7 / 3}}{g(z)^{2}} n^{\prime \prime}(z) \\
& +\frac{3 c}{2} \kappa \gamma \int d z \xi(z) \frac{\partial^{2}}{\partial z^{2}}\left(\frac{\xi(z) n(z)^{-4 / 3}}{g(z)^{2}}\right) \\
& +6 c \kappa \gamma^{2} \int d z \xi(z) \frac{\partial}{\partial z}\left(\frac{\xi(z) n^{\prime \prime}(z) n^{\prime}(z)}{n(z)^{4} g(z)^{3}}\right) \\
& +8 c \kappa \gamma^{2} \int d z \xi(z)^{2} \frac{n^{\prime \prime}(z)\left(n^{\prime}(z)\right)^{2}}{n(z)^{5} g(z)^{3}}
\end{aligned}
$$




\section{Appendix B}

\section{The PGG kernel for quasi-2DEGs}

In a quantum well of finite width, the single-particle orbitals have the form

$$
\varphi_{j}(\mathbf{r})=e^{i \mathbf{q}_{\|} \cdot \mathbf{r}_{\|}} \varphi_{j}(z)
$$

where we ignore the normalization factor $A^{-1 / 2}$ for simplicity. The PGG exchange kernel (2.24) becomes

$$
f_{\mathrm{x}}^{\mathrm{PGG}}\left(\mathbf{r}, \mathbf{r}^{\prime}\right)=-\frac{2}{\left|\mathbf{r}-\mathbf{r}^{\prime}\right| n(z) n\left(z^{\prime}\right)}\left|\sum_{j=1}^{N_{\text {occ }}} \varphi_{j}(z) \varphi_{j}\left(z^{\prime}\right) \sum_{\mathbf{k}_{\|}} \theta\left(k_{j}-k_{\|}\right) e^{i \mathbf{k}_{\|} \cdot\left(\mathbf{r}_{\|}-\mathbf{r}_{\|}^{\prime}||\right.}\right|^{2}
$$

where $k_{j}=\sqrt{2\left(\varepsilon_{F}-\varepsilon_{j}\right)}$. Carrying out the integral over $\mathbf{k}_{\|}$, and defining $\boldsymbol{\rho}_{\|}=\mathbf{r}_{\|}-\mathbf{r}_{\|}^{\prime}$, one finds

$$
f_{\mathrm{x}}^{\mathrm{PGG}}\left(\mathbf{r}, \mathbf{r}^{\prime}\right)=-\frac{2}{\left|\mathbf{r}-\mathbf{r}^{\prime}\right| n(z) n\left(z^{\prime}\right)}\left|\sum_{j=1}^{N_{\text {occ }}} \varphi_{j}(z) \varphi_{j}\left(z^{\prime}\right) \frac{k_{j} J_{1}\left(k_{j} \rho_{\|}\right)}{2 \pi \rho_{\|}}\right|^{2},
$$


where $J_{1}$ denotes a standard Bessel function. Fourier transformation with respect to $\boldsymbol{\rho}_{\| \text {yields }}$

$$
\begin{gathered}
f_{\mathrm{x}}^{\mathrm{PGG}}\left(q_{\|}, z, z^{\prime}\right)=-\sum_{j, l}^{N_{\mathrm{occ}}} k_{j} k_{l} \frac{\varphi_{j}(z) \varphi_{l}(z) \varphi_{j}\left(z^{\prime}\right) \varphi_{l}\left(z^{\prime}\right)}{\pi n(z) n\left(z^{\prime}\right)} \\
\times \int_{0}^{\infty} d \rho_{\|} \frac{J_{0}\left(q_{\|} \rho_{\|}\right) J_{1}\left(k_{j} \rho_{\|}\right) J_{1}\left(k_{l} \rho_{\|}\right)}{\rho_{\|} \sqrt{\rho_{\|}^{2}+\left(z-z^{\prime}\right)^{2}}}
\end{gathered}
$$

If only the first subband is occupied, this simplifies to

$$
f_{\mathrm{x}}^{\mathrm{PGG}}\left(q_{\|}, z, z^{\prime}\right)=-\frac{2}{N_{s}} \int_{0}^{\infty} d \rho_{\|} \frac{J_{0}\left(q_{\|} \rho_{\|}\right) J_{1}^{2}\left(k_{1} \rho_{\|}\right)}{\rho_{\|} \sqrt{\rho_{\|}^{2}+\left(z-z^{\prime}\right)^{2}}} .
$$

In the limit of a pure 2DEG, the PGG exchange kernel thus becomes

$$
f_{\mathrm{x}, 2 \mathrm{D}}^{\mathrm{PGG}}\left(q_{\|}\right)=-\frac{2}{n_{2 \mathrm{D}}} \int_{0}^{\infty} \frac{d \rho_{\|}}{\rho_{\|}^{2}} J_{0}\left(q_{\|} \rho_{\|}\right) J_{1}^{2}\left(k_{F}^{2 \mathrm{D}} \rho_{\|}\right) .
$$

Let us mention that the PGG exchange kernel (2.24) can also be written as

$$
f_{\mathrm{x}}^{\mathrm{PGG}}\left(\mathbf{r}, \mathbf{r}^{\prime}\right)=2 \frac{g_{0}\left(\mathbf{r}, \mathbf{r}^{\prime}\right)-1}{\left|\mathbf{r}-\mathbf{r}^{\prime}\right|}
$$

where $g_{0}\left(\mathbf{r}, \mathbf{r}^{\prime}\right)$ is the noninteracting pair correlation function. One then finds the following alternative form of the PGG exchange kernel for a 2DEG:

$$
f_{\mathrm{x}, 2 \mathrm{D}}^{\mathrm{PGG}}\left(q_{\|}\right)=-\frac{\pi}{q_{\|}} G_{\uparrow \uparrow}^{\mathrm{S}}\left(q_{\|}\right),
$$


where

$$
\begin{aligned}
G_{\uparrow \uparrow}^{\mathrm{S}}\left(q_{\|}\right) & =-\frac{q_{\|}}{2 \pi^{2} n} \int \frac{d^{2} q_{\|}^{\prime}}{\left|\mathbf{q}_{\|}-\mathbf{q}_{\|}^{\prime}\right|}\left[S_{0}\left(q_{\|}^{\prime}\right)-1\right] \\
& =-\frac{2 q_{\|}}{\pi^{2} n} \int_{0}^{\infty} \frac{q_{\|}^{\prime} d q_{\|}^{\prime}}{q_{\|}+q_{\|}^{\prime}} K\left(\frac{\sqrt{4 q_{\|} q_{\|}^{\prime}}}{q_{\|}+q_{\|}^{\prime}}\right)\left[S_{0}\left(q_{\|}^{\prime}\right)-1\right]
\end{aligned}
$$

is the so-called Slater local field factor $\left(S_{0}\right.$ is the noninteracting static structure factor and $K$ is the complete elliptic integral of the first kind).[2] 


\section{Appendix $\mathrm{C}$}

\section{ISTLS in the exchange-only limit}

In the inhomogeneous STLS (ISTLS) approach, the xc kernel has the following tensorial form: $[26,27]$

$$
f_{\mathbf{x c}, \mu \nu}^{\mathrm{ISTLS}}\left(\mathbf{r}, \mathbf{r}^{\prime}\right)=-\frac{2}{\omega^{2}}\left[g\left(\mathbf{r}, \mathbf{r}^{\prime}\right)-1\right] \frac{\partial}{\partial_{\mu}} \frac{1}{\left|\mathbf{r}-\mathbf{r}^{\prime}\right|} \frac{\partial}{\partial_{\nu}^{\prime}}
$$

where $\mu, \nu$ denote Cartesian coordinates and $g\left(\mathbf{r}, \mathbf{r}^{\prime}\right)$ is the pair correlation function. The exchange-only limit of this expression is obtained by using the noninteracting pair correlation function, which yields

$$
f_{\mathbf{x}, \mu \nu}^{\mathrm{ISTLS}}\left(\mathbf{r}, \mathbf{r}^{\prime}\right)=2 \frac{\left|\sum_{j=1}^{N_{\text {occ }}} \varphi_{j}(\mathbf{r}) \varphi_{j}^{*}\left(\mathbf{r}^{\prime}\right)\right|^{2}}{\omega^{2} n(\mathbf{r}) n\left(\mathbf{r}^{\prime}\right)} \frac{\partial}{\partial_{\mu}} \frac{1}{\left|\mathbf{r}-\mathbf{r}^{\prime}\right|} \frac{\partial}{\partial_{\nu}^{\prime}}
$$

We consider the case of a quantum well with finite width, where the Kohn-Sham orbitals have the form (B.1), and we limit ourselves to plasmon modes with in-plane wavevector $q_{\|}=0$, so that the dynamics is uniform within the plane of the well and, 
hence, effectively one-dimensional. Then, only the $z z$ component of the tensorial $\mathrm{xc}$ kernel is relevant, and it is straightforward to transform it to a scalar exchange kernel[29]. Using the same notation as in Appendix B, we obtain

$$
\begin{aligned}
f_{\mathrm{x}}^{\operatorname{ISTLS}}\left(q_{\|}=0, z, z^{\prime}\right) & =\int_{z}^{\infty} d z_{1} \int_{0}^{\infty} \frac{d \rho_{\|}}{\rho_{\|}} \frac{\left|\sum_{j}^{N_{\mathrm{occ}}} \varphi_{j}^{*}\left(z_{1}\right) \varphi_{j}\left(z^{\prime}\right) k_{j} J_{1}\left(k_{j} \rho_{\|}\right)\right|^{2}}{\pi n\left(z_{1}\right) n\left(z^{\prime}\right)} \\
& \times\left(\frac{\partial}{\partial z_{1}} \frac{1}{\sqrt{\rho_{\|}^{2}+\left(z_{1}-z^{\prime}\right)^{2}}}\right)
\end{aligned}
$$

Comparing with Eq. (B.4) [notice that $J_{0}(0)=1$ ], we can rewrite this as

$$
\begin{aligned}
f_{\mathrm{x}}^{\operatorname{ISTLS}}\left(0, z, z^{\prime}\right) & =f_{\mathrm{x}}^{\mathrm{PGG}}\left(0, z, z^{\prime}\right) \\
& -\int_{z}^{\infty} d z_{1} \int_{0}^{\infty} \frac{d \rho_{\|}}{\rho_{\|}} \sum_{l, m}^{N_{\text {occ }}} \frac{k_{l} k_{m} J_{1}\left(k_{l} \rho_{\|}\right) J_{1}\left(k_{m} \rho_{\|}\right)}{\sqrt{\rho_{\|}^{2}+\left(z_{1}-z^{\prime}\right)^{2}}} \\
& \times \frac{\partial}{\partial z_{1}}\left(\frac{\varphi_{l}\left(z_{1}\right) \varphi_{l}^{*}\left(z^{\prime}\right) \varphi_{m}^{*}\left(z_{1}\right) \varphi_{m}\left(z^{\prime}\right)}{\pi n\left(z_{1}\right) n\left(z^{\prime}\right)}\right)
\end{aligned}
$$

It thus turns out that the ISTLS exchange kernel is equal to the PGG exchange kernel plus a correction term. If only the lowest subband is occupied $\left(N_{\text {occ }}=1\right)$, the correction term vanishes because then the derivative with respect to $z_{1}$ gives zero.

Figure C.1 gives a comparison of PGG and ISTLS for the case of a quantum well with 5 occupied subbands. The figure shows the frequency-dependent photoabsorption cross section corresponding the intersubband charge plasmons. As can be seen, the difference between PGG and ISTLS is marginal. 


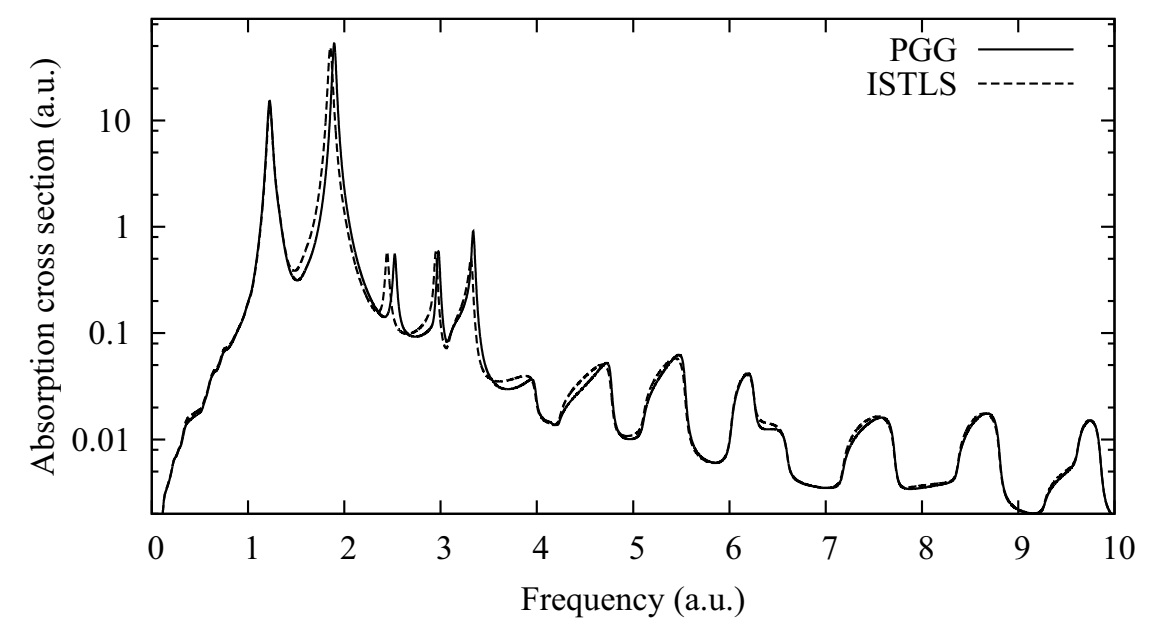

Figure C.1: Photoabsorption cross section for $q_{\|}=0$ intersubband charge plasmons, for a quantum well with 5 occupied subbands, comparing PGG and exchange-only ISTLS. 


\section{Appendix D}

\section{Derivation of Eq. (3.6)}

In this Appendix we provide the derivation of Eq. (3.6), using the linear-response formalism described in Sec. 3.3. The spin-flip response function, Eq. (3.49), is given by

$$
\chi_{\sigma \sigma^{\prime}, \tau \tau^{\prime}}\left(\mathbf{q}, y, y^{\prime}, \omega\right)=F_{\sigma \sigma^{\prime}, \tau \tau^{\prime}}(\mathbf{q}, \omega) \phi^{2}(y) \phi^{2}\left(y^{\prime}\right)
$$

where

$$
\begin{aligned}
F_{\sigma \sigma^{\prime}, \tau \tau^{\prime}}(\mathbf{q}, \omega)=-\sum_{p p^{\prime}}^{ \pm 1} \int \frac{d^{2} k}{(2 \pi)^{2}} \frac{f\left(E_{p \mathbf{k}}\right)}{\omega-E_{p \mathbf{k}}+E_{p^{\prime} \mathbf{k}-\mathbf{q}}+i \eta} \\
\\
{\left[\delta_{\sigma \uparrow} \psi_{\mathbf{k}}^{p \uparrow}+\delta_{\sigma \downarrow} \psi_{\mathbf{k}}^{p \downarrow}\right]\left[\delta_{\sigma^{\prime} \uparrow} \psi_{\mathbf{k}-\mathbf{q}}^{p^{\prime} \uparrow}+\delta_{\sigma^{\prime} \downarrow} \psi_{\mathbf{k}-\mathbf{q}}^{p^{\prime}}\right] } \\
{\left[\delta_{\tau \uparrow} \psi_{\mathbf{k}}^{p \uparrow}+\delta_{\tau \downarrow} \psi_{\mathbf{k}}^{p \downarrow}\right]\left[\delta_{\tau^{\prime} \uparrow} \psi_{\mathbf{k}-\mathbf{q}}^{p^{\prime} \uparrow}+\delta_{\tau^{\prime} \downarrow} \psi_{\mathbf{k}-\mathbf{q}}^{p^{\prime} \downarrow}\right] } \\
+\sum_{p p^{\prime}}^{ \pm 1} \int \frac{d^{2} k}{(2 \pi)^{2}} \frac{f\left(E_{p \mathbf{k}}\right)}{\omega+E_{p \mathbf{k}}-E_{p^{\prime} \mathbf{k}+\mathbf{q}}+i \eta} \\
{\left[\delta_{\sigma \uparrow \uparrow} \psi_{\mathbf{k}+\mathbf{q}}^{p^{\prime} \uparrow}+\delta_{\sigma \downarrow} \psi_{\mathbf{k}+\mathbf{q}}^{p^{\prime} \downarrow}\right]\left[\delta_{\sigma^{\prime} \uparrow} \psi_{\mathbf{k}}^{p \uparrow}+\delta_{\sigma^{\prime} \downarrow} \psi_{\mathbf{k}}^{p \downarrow}\right] } \\
\\
{\left[\delta_{\tau \uparrow} \psi_{\mathbf{k}+\mathbf{q}}^{p^{\prime} \uparrow}+\delta_{\tau \downarrow} \psi_{\mathbf{k}+\mathbf{q}}^{p^{\prime} \downarrow}\right]\left[\delta_{\tau^{\prime} \uparrow} \psi_{\mathbf{k}}^{p \uparrow}+\delta_{\tau^{\prime} \downarrow} \psi_{\mathbf{k}}^{p \downarrow}\right] . }
\end{aligned}
$$


To second order in SOC, the energy eigenvalues (3.40) are given by

$$
E_{p \mathbf{k}}=\frac{k^{2}}{2}+\frac{\varepsilon_{\uparrow}+\varepsilon_{\downarrow}}{2}+\frac{p}{2}\left(Z^{*}-\mathbf{k} \cdot \mathbf{q}_{0}+\frac{\left(\mathbf{k} \cdot \mathbf{q}_{1}\right)^{2}}{2 Z^{*}}\right),
$$

where $\mathbf{q}_{0}$ and $\mathbf{q}_{1}$ are defined in Eqs. (3.35)-(3.38), which leads to

$$
\begin{aligned}
& \mathbf{k} \cdot \mathbf{q}_{0}=2 k\left[\alpha \cos \left(\varphi-\varphi_{k}\right)+\beta \sin \left(\varphi+\varphi_{k}\right)\right] \\
& \mathbf{k} \cdot \mathbf{q}_{1}=-2 k\left[\alpha \sin \left(\varphi-\varphi_{k}\right)-\beta \cos \left(\varphi+\varphi_{k}\right)\right] .
\end{aligned}
$$

The single-particle states (3.46) and (3.47) are given to second order in SOC by

$$
\begin{aligned}
& \psi_{+}=\left(\begin{array}{c}
1-\frac{\left(\mathbf{k} \cdot \mathbf{q}_{1}\right)^{2}}{8 Z^{* 2}} \\
\frac{\mathbf{k} \cdot \mathbf{q}_{1}}{2 Z^{*}}+\frac{\left(\mathbf{k} \cdot \mathbf{q}_{0}\right)\left(\mathbf{k} \cdot \mathbf{q}_{1}\right)}{2 Z^{* 2}}
\end{array}\right) \phi(y) \\
& \psi_{-}=\left(\begin{array}{c}
-\frac{\mathbf{k} \cdot \mathbf{q}_{1}}{2 Z^{*}}-\frac{\left(\mathbf{k} \cdot \mathbf{q}_{0}\right)\left(\mathbf{k} \cdot \mathbf{q}_{1}\right)}{2 Z^{* 2}} \\
1-\frac{\left(\mathbf{k} \cdot \mathbf{q}_{1}\right)^{2}}{8 Z^{* 2}}
\end{array}\right) \phi(y) .
\end{aligned}
$$

In the following, we use the abbreviation $h_{1 \mathbf{k}}=\mathbf{k} \cdot \mathbf{q}_{1} / Z^{*}$.

We are interested in the spin-flip waves for small $q$. The response function (D.2) at $q=0$ can be written in the following way:

$$
\begin{aligned}
\underline{\underline{F}}(0, \omega)= & -\int \frac{d^{2} k}{(2 \pi)^{2}} \frac{f\left(E_{+\mathbf{k}}\right)}{\omega-E_{+\mathbf{k}}+E_{-\mathbf{k}}+i \eta} \underline{\underline{R}}^{+} \\
& +\int \frac{d^{2} k}{(2 \pi)^{2}} \frac{f\left(E_{+\mathbf{k}}\right)}{\omega+E_{+\mathbf{k}}-E_{-\mathbf{k}}+i \eta} \underline{\underline{R}}^{-} \\
& -\int \frac{d^{2} k}{(2 \pi)^{2}} \frac{f\left(E_{-\mathbf{k}}\right)}{\omega-E_{-\mathbf{k}}+E_{+\mathbf{k}}+i \eta} \underline{\underline{R}}^{-} \\
& +\int \frac{d^{2} k}{(2 \pi)^{2}} \frac{f\left(E_{-\mathbf{k}}\right)}{\omega+E_{-\mathbf{k}}-E_{+\mathbf{k}}+i \eta} \underline{R}^{+}
\end{aligned}
$$


where the matrices $\underline{\underline{R}}^{+}$and $\underline{\underline{R}}^{-}$are given by

$$
\begin{array}{r}
\underline{\underline{R}}^{+}=\left(\begin{array}{cccc}
h_{1 \mathrm{k}}^{2} & -h_{1 \mathrm{k}} & 0 & -h_{1 \mathrm{k}}^{2} \\
-h_{1 \mathrm{k}} & 1-2 h_{1 \mathrm{k}}^{2} & -h_{1 \mathrm{k}}^{2} & h_{1 \mathrm{k}} \\
0 & -h_{1 \mathrm{k}}^{2} & 0 & 0 \\
-h_{1 \mathrm{k}}^{2} & h_{1 \mathrm{k}} & 0 & h_{1 \mathrm{k}}^{2}
\end{array}\right) \\
\underline{\underline{R}}^{-}=\left(\begin{array}{cccc}
h_{1 \mathrm{k}}^{2} & 0 & -h_{1 \mathrm{k}} & -h_{1 \mathrm{k}}^{2} \\
0 & 0 & -h_{1 \mathrm{k}}^{2} & 0 \\
-h_{1 \mathrm{k}} & -h_{1 \mathrm{k}}^{2} & 1-2 h_{1 \mathrm{k}}^{2} & h_{1 \mathrm{k}} \\
-h_{1 \mathrm{k}}^{2} & 0 & h_{1 \mathrm{k}} & h_{1 \mathrm{k}}^{2}
\end{array}\right) .
\end{array}
$$

Now let us calculate the energy in the denominator and drop the $i \eta$. We have

$$
\begin{aligned}
\underline{\underline{F}}(0, \omega) & =\int \frac{d^{2} k}{(2 \pi)^{2}} \frac{f\left(E_{+\mathbf{k}}\right)-f\left(E_{-\mathbf{k}}\right)}{\omega-Z^{*}+g_{0}-g_{1}} \underline{R}^{+} \\
& +\int \frac{d^{2} k}{(2 \pi)^{2}} \frac{f\left(E_{+\mathbf{k}}\right)-f\left(E_{-\mathbf{k}}\right)}{\omega+Z^{*}-g_{0}+g_{1}} \underline{R}^{-} \\
& =\frac{\underline{\underline{R}}^{+}}{\omega-Z^{*}} \int \frac{d^{2} k}{(2 \pi)^{2}} \frac{f\left(E_{-\mathbf{k}}\right)-f\left(E_{+\mathbf{k}}\right)}{1+\frac{g_{0}-g_{1}}{\omega-Z^{*}}} \\
& +\frac{\underline{\underline{R}}^{-}}{\omega+Z^{*}} \int \frac{d^{2} k}{(2 \pi)^{2}} \frac{f\left(E_{+\mathbf{k}}\right)-f\left(E_{-\mathbf{k}}\right)}{1+\frac{-g_{0}+g_{1}}{\omega+Z^{*}}}
\end{aligned}
$$

where we abbreviate

$$
g_{0}=2 \mathbf{k} \cdot \mathbf{q}_{0}, \quad g_{1}=\frac{2\left(\mathbf{k} \cdot \mathbf{q}_{1}\right)^{2}}{Z^{*}} .
$$

Next, we expand the integrands of $F_{\sigma \sigma^{\prime}, \tau \tau^{\prime}}(0, \omega)$ up to second order in SOC, and carry 
out the integration over $\mathbf{k}$ for each element of the $4 \times 4$ matrices $\underline{\underline{R}}^{+}$and $\underline{R}^{-}$. We use a notation where $F_{0}^{ \pm}, F_{1}^{ \pm}$, and $F_{2}^{ \pm}$come from those terms containing zeroth, first and second order in $h_{1 \mathbf{k}}$, respectively. After a lengthy calculation, the result is

$$
\begin{aligned}
& \underline{\underline{F}}(0, \omega)= \\
& \left(\begin{array}{cccc}
F_{2}^{+}+F_{2}^{-} & -F_{1}^{+} & -F_{1}^{-} & -F_{2}^{+}-F_{2}^{-} \\
-F_{1}^{+} & F_{0}^{+}-2 F_{2}^{+} & -F_{2}^{+}-F_{2}^{-} & F_{1}^{+} \\
-F_{1}^{-} & -F_{2}^{+}-F_{2}^{-} & F_{0}^{-}-2 F_{2}^{-} & F_{1}^{-} \\
-F_{2}^{+}-F_{2}^{-} & F_{1}^{+} & F_{1}^{-} & F_{2}^{+}+F_{2}^{-}
\end{array}\right)
\end{aligned}
$$

where

$$
\begin{aligned}
F_{0}^{ \pm} & =\frac{ \pm Z^{*}}{2 \pi\left(\omega \mp Z^{*}\right)} \pm \frac{N_{s}(a-b)}{Z^{*}\left(\omega \mp Z^{*}\right)}+\frac{2 N_{s}(a+b)}{\left(\omega \mp Z^{*}\right)^{2}} \\
& +\frac{N_{s}(a-b)}{\left(\omega \mp Z^{*}\right)^{2}} \pm \frac{2 N_{s} Z^{*}(a+b)}{\left(\omega \mp Z^{*}\right)^{3}} \\
F_{1}^{ \pm} & =\mp c N_{s}\left[\frac{1}{Z^{*}\left(\omega \mp Z^{*}\right)} \pm \frac{1}{\left(\omega \mp Z^{*}\right)^{2}}\right] \\
F_{2}^{ \pm} & =\frac{ \pm N_{s}(a-b)}{2 Z^{*}\left(\omega \mp Z^{*}\right)}
\end{aligned}
$$

and $a=\alpha^{2}+\beta^{2}, b=2 \alpha \beta \sin 2 \varphi$ and $c=2 \alpha \beta \cos (2 \varphi)$.

Instead of the spin-density-matrix response, we will work with density-magnetization response, Eq. (3.52). Further details of the transformation can be found in the Appendix of Ref. [68]. It follows that all contributions to the density channel vanish, and the remaining nonvanishing terms of the density-magnetization response 
function are

$$
\begin{aligned}
& \Pi_{11}=\chi_{\uparrow \downarrow \uparrow \downarrow}+\chi_{\uparrow \downarrow \downarrow \uparrow}+\chi_{\downarrow \uparrow \uparrow \downarrow}+\chi_{\downarrow \uparrow \downarrow \uparrow} \\
& \Pi_{12}=-i\left(\chi_{\uparrow \downarrow \uparrow \downarrow}-\chi_{\uparrow \downarrow \downarrow \uparrow}+\chi_{\downarrow \uparrow \uparrow \downarrow}-\chi_{\downarrow \uparrow \downarrow \uparrow}\right) \\
& \Pi_{13}=\chi_{\uparrow \downarrow \uparrow \uparrow}-\chi_{\uparrow \downarrow \downarrow \downarrow}+\chi_{\downarrow \uparrow \uparrow \uparrow}-\chi_{\downarrow \uparrow \downarrow \downarrow} \\
& \Pi_{21}=i\left(\chi_{\uparrow \downarrow \uparrow \downarrow}+\chi_{\uparrow \downarrow \downarrow \uparrow}-\chi_{\downarrow \uparrow \uparrow \downarrow}-\chi_{\downarrow \uparrow \downarrow \uparrow}\right) \\
& \Pi_{22}=\chi_{\uparrow \downarrow \uparrow \downarrow}-\chi_{\uparrow \downarrow \downarrow \uparrow}-\chi_{\downarrow \uparrow \uparrow \downarrow}+\chi_{\downarrow \uparrow \downarrow \uparrow} \\
& \Pi_{23}=i\left(\chi_{\uparrow \downarrow \uparrow \uparrow}-\chi_{\uparrow \downarrow \downarrow \downarrow}-\chi_{\downarrow \uparrow \uparrow \uparrow}+\chi_{\downarrow \uparrow \downarrow \downarrow}\right) \\
& \Pi_{31}=\chi_{\uparrow \uparrow \uparrow \downarrow}+\chi_{\uparrow \uparrow \downarrow \uparrow}-\chi_{\downarrow \downarrow \uparrow \downarrow}-\chi_{\downarrow \downarrow \downarrow \uparrow} \\
& \Pi_{32}=-i\left(\chi_{\uparrow \uparrow \uparrow \downarrow}-\chi_{\uparrow \uparrow \downarrow \uparrow}-\chi_{\downarrow \downarrow \uparrow \downarrow}+\chi_{\downarrow \downarrow \downarrow \uparrow}\right) \\
& \Pi_{33}=\chi_{\uparrow \uparrow \uparrow \uparrow}-\chi_{\uparrow \uparrow \downarrow \downarrow}-\chi_{\downarrow \downarrow \uparrow \uparrow}+\chi_{\downarrow \downarrow \downarrow \downarrow}
\end{aligned}
$$

and $\Pi_{00}=\Pi_{01}=\Pi_{02}=\Pi_{03}=\Pi_{10}=\Pi_{20}=\Pi_{30}=0$. Therefore, the total response function is a $4 \times 4$ matrix whose elements defined as follows:

$$
\begin{aligned}
& \Pi_{11}=F_{0}^{+}+F_{0}^{-}-4\left(F_{2}^{-}+F_{2}^{+}\right) \\
& \Pi_{12}=-i\left(F_{0}^{+}-F_{0}^{-}-2 F_{2}^{+}+2 F_{2}^{-}\right) \\
& \Pi_{13}=-2\left(F_{1}^{+}+F_{1}^{-}\right) \\
& \Pi_{21}=i\left(F_{0}^{+}-F_{0}^{-}-2 F_{2}^{+}+2 F_{2}^{-}\right) \\
& \Pi_{22}=F_{0}^{+}+F_{0}^{-} \\
& \Pi_{23}=2 i\left(F_{1}^{-}-F_{1}^{+}\right)
\end{aligned}
$$




$$
\begin{aligned}
& \Pi_{31}=-2\left(F_{1}^{+}+F_{1}^{-}\right) \\
& \Pi_{32}=-2 i\left(F_{1}^{-}-F_{1}^{+}\right) \\
& \Pi_{33}=4\left(F_{2}^{+}+F_{2}^{-}\right)
\end{aligned}
$$

where each element is multiplied with $\phi^{2}(y) \phi^{2}\left(y^{\prime}\right)$. In order to find the collective modes, we need to determine those frequencies where the matrix

$$
\underline{\underline{M}}(\mathbf{q}, \omega)=\underline{\underline{H}}^{\mathrm{xc}}(\mathbf{q}, \omega) \underline{\underline{\Pi}}(\mathbf{q}, \omega)
$$

has the eigenvalue 1, where the xc matrix $\underline{\underline{H}}^{\mathrm{xc}}$ is given by Eq. (3.54). In other words, we solve the $4 \times 4$ eigenvalue problem

$$
\underline{\underline{M}}(\mathbf{q}, \omega) \vec{x}=\lambda(\mathbf{q}, \omega) \vec{x}
$$

and find the mode frequencies by solving $\lambda(\mathbf{q}, \omega)=1$ for $\omega$, where $\mathbf{q}$ is fixed. Since here our goal is to obtain the coefficient $E_{0}$ to second order in the Rashba and Dresselhaus coupling strengths $\alpha$ and $\beta$, we carry out a perturbative expansion of the eigenvalue problem (D.18) in orders of spin-orbit coupling. At $q=0$, the matrix can be written as

$$
\underline{\underline{M}}(0, \omega)=\underline{\underline{M}}^{(0)}+\underline{\underline{M}}^{(2)}+\ldots
$$

where superscripts indicate the order of spin-orbit coupling (the linear order vanishes at $q=0)$.

We now write $\underline{\underline{\Pi}}=\underline{\underline{\Pi}}^{(0)}+\underline{\underline{\Pi}}^{(2)}$, where $\underline{\underline{\Pi}}^{(0)}$ and $\underline{\underline{\Pi}}^{(2)}$ are in zero and second order 
in spin-orbit coupling, respectively. Let us first work out the zero-order case and solve the zero-order eigenvalue problem $\underline{\underline{M}}^{(0)} \vec{x}^{(0)}=\lambda^{(0)} \vec{x}^{(0)}$. The zero-order response function matrix is

$$
\underline{\underline{\Pi}}^{(0)}=\frac{Z^{*} \phi^{2}(y) \phi^{2}\left(y^{\prime}\right)}{\pi\left(\omega^{2}-Z^{*^{2}}\right)}\left(\begin{array}{cccc}
0 & 0 & 0 & 0 \\
0 & Z^{*} & -i \omega & 0 \\
0 & i \omega & Z^{*} & 0 \\
0 & 0 & 0 & 0
\end{array}\right)
$$

Now we need to do the multiplication with the xc kernel matrix (see Eq. (3.60)):

$$
\underline{\underline{M}}^{(0)}=\frac{Z^{*} / \pi}{\omega^{2}-Z^{*}}\left(\begin{array}{cccc}
h_{00}^{\mathrm{xc}} & 0 & 0 & h_{03}^{\mathrm{xc}} \\
0 & h_{11}^{\mathrm{xc}} & 0 & 0 \\
0 & 0 & h_{22}^{\mathrm{xc}} & 0 \\
h_{30}^{\mathrm{xc}} & 0 & 0 & h_{33}^{\mathrm{xc}}
\end{array}\right)\left(\begin{array}{cccc}
0 & 0 & 0 & 0 \\
0 & Z^{*} & -i \omega & 0 \\
0 & i \omega & Z^{*} & 0 \\
0 & 0 & 0 & 0
\end{array}\right)
$$

The elements of the xc matrix, $h_{\mathrm{xc}}^{i j}$, are given in Eqs. (3.55)-(3.58), averaged over $\phi^{4}(y)$. In particular, we find $h_{11}^{\mathrm{xc}}=h_{22}^{\mathrm{xc}}=\pi f_{T}$, see Eq. (3.63). When we work this out, we find

$$
\underline{\underline{M}}^{(0)}=\frac{Z^{*} f_{T}}{\omega^{2}-Z^{* 2}}\left(\begin{array}{cccc}
0 & 0 & 0 & 0 \\
0 & Z^{*} & -i \omega & 0 \\
0 & i \omega & Z^{*} & 0 \\
0 & 0 & 0 & 0
\end{array}\right)
$$


The spin-flip wave at $q=0$ is at that frequency where the $4 \times 4$ matrix $\underline{\underline{M}}^{(0)}$ has eigenvalue 1. Working out the determinant leads to the following result:

$$
\omega_{0}=Z^{*}+Z^{*} f_{T}=Z
$$

(there is also a solution with a negative frequency, which we discard). We substitute $\omega_{0}$ back into Eq. (D.22), and end up with

$$
\underline{\underline{M}}^{(0)}=\left(\begin{array}{cccc}
0 & 0 & 0 & 0 \\
0 & \frac{1}{2+f_{T}} & -i \frac{1+f_{T}}{2+f_{T}} & 0 \\
0 & i \frac{1+f_{T}}{2+f_{T}} & \frac{1}{2+f_{T}} & 0 \\
0 & 0 & 0 & 0
\end{array}\right) .
$$

The normalized eigenvector which makes the eigenvalue of $\underline{\underline{M}}^{(0)}$ equal to 1 is

$$
\vec{x}^{(0)}=\frac{1}{\sqrt{2}}\left(\begin{array}{c}
0 \\
-i \\
1 \\
0
\end{array}\right) \text {. }
$$

To obtain the change of the eigenmodes caused by the presence of SOC, we need to determine $\lambda^{(2)}$. In perturbation theory, we obtain the second-order correction of the eigenvalues as

$$
\lambda^{(2)}=\left[\vec{x}^{(0)}\right]^{\dagger} \underline{\underline{M}}^{(2)} \vec{x}^{(0)},
$$


where we can construct $\underline{\underline{M}}^{(2)}$ by using $\underline{\underline{\Pi}}^{(2)}$ :

$$
\underline{\underline{M}}^{(2)}=\left(\begin{array}{cccc}
0 & 0 & 0 & 0 \\
0 & h_{11}^{\mathrm{xc}} \Pi_{11}^{(2)} & h_{11}^{\mathrm{xc}} \Pi_{12}^{(2)} & h_{11}^{\mathrm{xc}} \Pi_{13}^{(2)} \\
0 & h_{22}^{\mathrm{xc}} \Pi_{21}^{(2)} & h_{22}^{\mathrm{xc}} \Pi_{22}^{(2)} & h_{22}^{\mathrm{xc}} \Pi_{23}^{(2)} \\
0 & h_{33}^{\mathrm{xc}} \Pi_{31}^{(2)} & h_{33}^{\mathrm{xc}} \Pi_{32}^{(2)} & h_{33}^{\mathrm{xc}} \Pi_{33}^{(2)}
\end{array}\right)
$$

With the substitution of the terms in second-order in $\alpha$ and $\beta$ in the spin-flip response matrix, $\lambda^{(2)}$ in Eq. (D.26) will be

$$
\begin{aligned}
\lambda^{(2)}= & \frac{\pi f_{T}}{2}\left(\Pi_{11}^{(2)}+\Pi_{22}^{(2)}+i \Pi_{12}^{(2)}-i \Pi_{21}^{(2)}\right) \\
= & \pi f_{T}\left(2 F_{0}^{+}-4 F_{2}^{+}\right) \\
= & \frac{4 \pi N_{s} f_{T}(a+b)}{\left(\omega-Z^{*}\right)^{2}}+\frac{2 \pi N_{s} f_{T}(a-b)}{\left(\omega-Z^{*}\right)^{2}} \\
& +\frac{4 \pi N_{s} f_{T} Z^{*}(a+b)}{\left(\omega-Z^{*}\right)^{3}} .
\end{aligned}
$$

To remain within second order of SOC, we substitute $\omega_{0}$ in Eq. (D.23) back into $\lambda^{(2)}$, and get

$$
\begin{aligned}
\lambda^{(2)} & =\frac{6 \pi N_{s} a}{Z^{* 2} f_{T}}+\frac{2 \pi N_{s} b}{Z^{* 2} f_{T}}+\frac{4 \pi N_{s} Z^{*}(a+b)}{Z^{* 3} f_{T}^{2}} \\
& =\frac{2 \pi N_{s}}{Z^{* 2} f_{T}^{2}}\left[\left(\alpha^{2}+\beta^{2}\right)\left(3 f_{T}+2\right)+2 \alpha \beta \sin (2 \varphi)\left(f_{T}+2\right)\right]
\end{aligned}
$$

The condition for the spin wave at $q=0$ is that the eigenvalue is equal to 1 , so to 
second order perturbation theory we have

$$
1=\lambda^{(0)}+\lambda^{(2)}
$$

where $\lambda^{(0)}$ is known, so

$$
1=\frac{Z^{*} f_{T}}{\omega-Z^{*}}+\lambda^{(2)}
$$

which gives

$$
\omega-Z^{*}=Z^{*} f_{T}+\lambda^{(2)}\left(\omega-Z^{*}\right) .
$$

To lowest order in SOC, we replace $\omega$ on the right-hand side by $\omega_{0}$ :

$$
\omega=Z^{*}+Z^{*} f_{T}+\lambda^{(2)}\left(\omega_{0}-Z^{*}\right)
$$

and using $\omega_{0}=Z^{*}+Z^{*} f_{T}$ we obtain

$$
\omega=\omega_{0}+\lambda^{(2)} Z^{*} f_{T}
$$

Using expression (D.29), we obtain the final result

$$
E_{0}=Z+\frac{2 \pi N_{s}}{Z^{*} f_{T}}\left[\left(\alpha^{2}+\beta^{2}\right)\left(3 f_{T}+2\right)+2 \alpha \beta \sin (2 \varphi)\left(f_{T}+2\right)\right]
$$

which is given as Eq. (3.6). 


\section{Appendix E}

\section{In-plane anisotropy of the g-factor}

In this Appendix we consider the additional contributions to the angular modulation

of the spin-wave dispersion due to an in-plane anisotropy of the g-factor of the form $g_{x y} \sin (2 \varphi)$, where $g_{x y}$ is the off-diagonal component of the $g$ tensor $[93,94,95]$.

We have determined the magnitude of $g_{x y}$ experimentally in our CdMnTe quantum well sample by measuring the modulation amplitude of $E_{0}(\varphi)$ as a function of applied magnetic field $B$, for field strengths around $2 \mathrm{~T}$, and found a linear behavior. From the slope, we extracted a value of $g_{x y}=0.024$.

$g_{x y}$ can also be calculated using the following formula [93]:

$$
g_{x y}=\frac{2 \gamma}{\mu_{B}} \frac{e}{\hbar}\left(\left\langle k_{z}^{2}\right\rangle\langle z\rangle-\left\langle k_{z}^{2} z\right\rangle\right),
$$

with $\gamma=43.9 \mathrm{eV} \AA^{3}$ from $\mathbf{k} \cdot \mathbf{p}$ theory [5] in CdTe, and expressions like $\left\langle k_{z}^{2}\right\rangle$ are first quantized level wave function averages, where the wave function is deduced from a Schrödinger-Poisson algorithm. Our calculated value, $g_{x y}=0.037$ for an electron 
density of $2.6 \times 10^{11} \mathrm{~cm}^{-2}$, is in good agreement with the experimentally determined value for $g_{x y}$.

Similarly, the Rashba coefficient $\alpha$ can be calculated as $\alpha=r_{41}^{6 c 6 c} e\left\langle E_{z}\right\rangle$, with $e$ the electronic charge and $\left\langle E_{z}\right\rangle$ the wave-function averaged electric field along the growth axis deduced from a Schrödinger-Poisson algorithm, assuming that the electrons experience the delta-doping layer as an infinite sheet of positive charge, and using $r_{41}^{6 c 6 c}=6.93 \AA^{2}$ calculated by $\mathbf{k} \cdot \mathbf{p}$ perturbation theory [5] for CdTe. The Dresselhaus coefficient $\beta$ can be calculated as $\beta=\gamma\left\langle k_{z}^{2}\right\rangle$. We used $\gamma=43.9 \mathrm{eV} \AA^{3}$ from $\mathbf{k} \cdot \mathbf{p}$ theory [5] and estimated $\left\langle k_{z}^{2}\right\rangle$ from the Schrödinger-Poisson algorithm. Note that the same coefficient $\gamma$ determines both $g_{x y}$ and $\beta$.

The g-factor anisotropy will add extra terms to the Kohn-Sham single-particle Hamiltonian in Section 3.2. Specifically, the off-diagonal parts of the Kohn-Sham Hamiltonian in Eq. (3.31) in the reference frame $\mathcal{R}^{\prime}$ (see Fig. 3.1) become [93, 94, 95]

$$
\begin{aligned}
& h_{x^{\prime}}=-\left(\frac{Z}{2}+v_{\mathrm{xc}}^{-}\left(z^{\prime}\right)\right) \sin \varphi+\alpha k_{y^{\prime}}+\beta k_{x^{\prime}}+\frac{1}{2} g_{x y} \mu_{B} B \cos \varphi \\
& h_{y^{\prime}}=\left(\frac{Z}{2}+v_{\mathrm{xc}}^{-}\left(z^{\prime}\right)\right) \cos \varphi-\alpha k_{x^{\prime}}-\beta k_{y^{\prime}}-\frac{1}{2} g_{x y} \mu_{B} B \sin \varphi .
\end{aligned}
$$

Now we need to transform into the reference system $\mathcal{R}$, where the magnetic field is along the $z$-axis. With the additional anisotropy terms, Eq. (3.39) transforms into:

$$
\left[h_{0} \hat{\sigma}_{0}+\left(\frac{Z-\mathbf{k} \cdot \mathbf{q}_{0}-g_{x y} \mu_{B} B \sin (2 \varphi)}{2}+v_{\mathrm{xc}}^{-}\right) \hat{\sigma}_{z}+\frac{\mathbf{k} \cdot \mathbf{q}_{1}}{2} \hat{\sigma}_{x}\right] \psi_{p \mathbf{k}}=E_{p \mathbf{k}} \psi_{p \mathbf{k}}
$$

It immediately follows that the Kohn-Sham eigenvalues $E_{p \mathbf{k}}$ and eigenfunctions $\psi_{p \mathbf{k}}$ are the same as Eqs. (3.40)-(3.43), with $Z^{*}$ replaced by $Z^{*}-g_{x y} \mu_{B} B \sin (2 \varphi)$. The TDDFT linear response formalism then goes through as before, and the analytical 
Table E.1: Parameters to reproduce experimental spin-wave dispersions. "ALDA": $Z^{*}$ is calculated using the ALDA, and $\alpha$ and $\beta$ are fitted to reproduce $E_{0}$ and $E_{1}$. "full": $\alpha, \beta$ and $Z^{*}$ are fitted to reproduce $E_{0}, E_{1}$ and $E_{2}$.

\begin{tabular}{lcccc} 
& & $\alpha(\mathrm{mev} \AA)$ & $\beta(\mathrm{meV} \AA)$ & $Z^{*}(\mathrm{meV})$ \\
\hline$g_{x y}=0$ & ALDA & 1.6 & 3.1 & 0.57 \\
$g_{x y}=0.024$ & ALDA & 1.4 & 3.0 & 0.57 \\
\hline$g_{x y}=0$ & full & 2.2 & 3.9 & 0.63 \\
$g_{x y}=0.024$ & full & 2.1 & 3.5 & 0.63
\end{tabular}

formulas for $E_{0}$ and $E_{1}$, Eqs. (3.5) and (3.6), are unchanged, apart from the replacement of $Z^{*}$ by $Z^{*}-g_{x y} \mu_{B} B \sin (2 \varphi)$ in Eqs. (3.5) and (3.6) and the replacement of $Z$ by $Z-\left(1+f_{T}\right) g_{x y} \mu_{B} B \sin (2 \varphi)$ in Eq. (3.6).

In Figs. E.1 and E.2, the experimental data for $E_{0}(\varphi), E_{1}(\varphi)$, and $E_{2}(\varphi)$ is compared with the theory that includes the $g_{x y}$ anisotropy, using the same fitting procedures as in Figs. 3.5 and 3.6. We find that the contributions of the $g_{x y}$ anisotropy are very small, and we obtain fits of the same quality as before, with fitted parameters $\left(\alpha, \beta\right.$, and $\left.Z^{*}\right)$ that differ only slightly from those obtained without $g_{x y}$.

The results are summarized in Table E.1. 

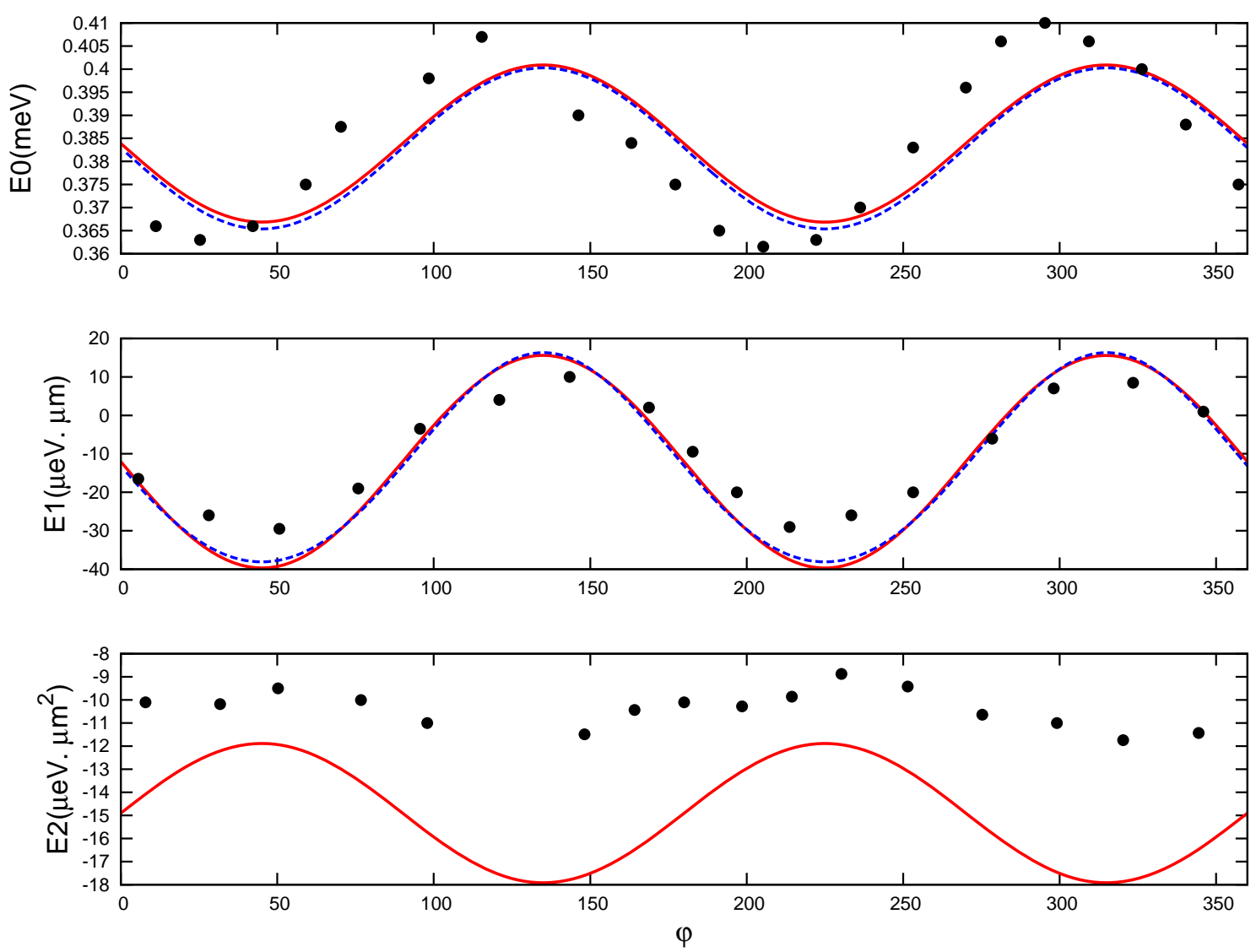

Figure E.1: Coefficients $E_{0}, E_{1}$, and $E_{2}$ of the spin-wave dispersion, Eq. (3.4), as a function of angle $\varphi$. Dots: experimental data. Lines: theoretical results with $g_{x y}=0.024$, using $Z^{*}=0.569 \mathrm{meV}$ obtained with ALDA, and $\alpha=1.4 \mathrm{meV} \AA$ and $\beta=3.0 \mathrm{meV} \AA$ obtained by fitting $E_{0}$ and $E_{1}$. The red lines follow from the fully numerical solution of Eq. (3.60), the dashed blue lines follow from the analytical formulas (3.6) and (3.5). 

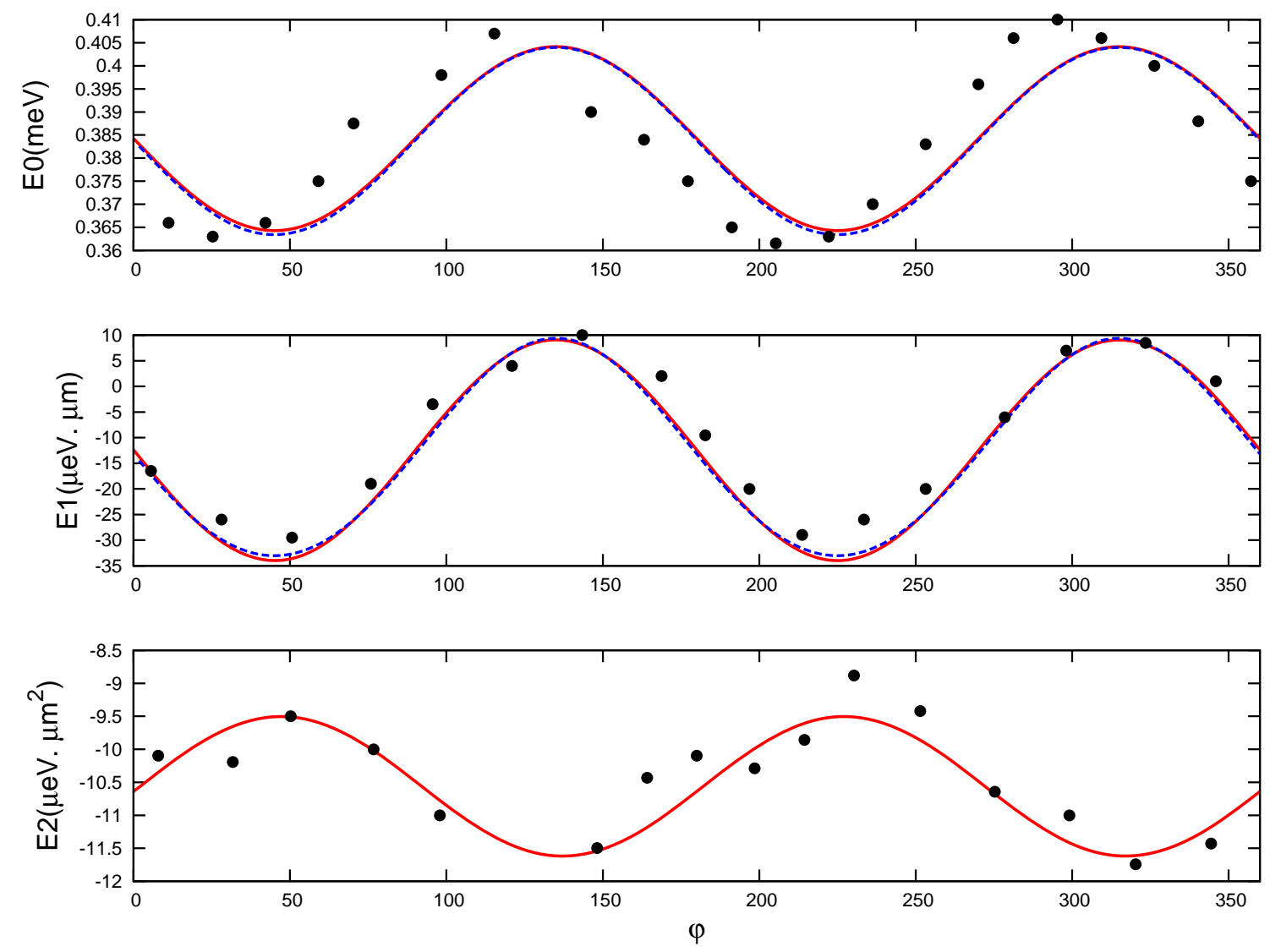

Figure E.2: Same as Fig. E.1, but using $Z^{*}=0.63 \mathrm{meV}, \alpha=2.1 \mathrm{meV} \AA$, and $\beta=3.5$ meVA obtained from a best fit to the experimental data. 


\section{Bibliography}

[1] T. Ando, A. B. Fowler, and F. Stern, Rev. Mod. Phys. 54, 437 (1982).

[2] G. F. Giuliani and G. Vignale, Quantum Theory of the Electron Liquid (Cambridge University Press, 2005).

[3] J. H. Davies, The Physics of Low-Dimensional Semiconductors (Cambridge University Press, 1998).

[4] P. Harrison, Quantum wells, wires and dots, 2nd Edition (Wiley, Chichester, 2005).

[5] R. Winkler, Spin-orbit coupling effects in two-dimensional electron and hole systems, vol. 191 of Springer Tracts in Modern Physics (Springer, Berlin, 2003).

[6] D. Bercioux and P. Lucignano, Rep. Prog. Phys. 78, 106001 (2015).

[7] I. Žutić, J. Fabian, and S. Das Sarma, Rev. Mod. Phys. 76, 323 (2004).

[8] A. H. Castro Neto, F. Guinea, N. M. R. Peres, K. S. Novoselov, and A. K. Geim, Rev. Mod. Phys. 81, 109 (2009).

[9] C. L. Kane and E. J. Mele, Phys. Rev. Lett. 95, 146802 (2005). 
[10] C. L. Kane and E. J. Mele, Phys. Rev. Lett. 95, 226801 (2005).

[11] B. A. Bernevig and T. L. Hughes, Topological insulators and topological superconductors (Princeton University Press, 2013).

[12] T. Moriya, Spin fluctuations in itinerant electron magnetism (Springer, Berlin, 2012).

[13] V. V. Kruglyak, S. O. Demokritov, and D. Grundler, J. Phys. D: Appl. Phys. 43, 264001 (2010).

[14] A. V. Chumak, V. I. Vasyuchka, A. A. Serga, and B. Hillebrands, Nature Phys. 11, 453 (2015).

[15] Y. Kajiwara, K. Harii, S. Takahashi, J. Ohe, K. Uchida, M. Mizuguchi, H. Umezawa, H. Kawai, K. Ando, K. Takanashi, S. Maekawa, and E. Saitoh, Nature 464, 262 (2010).

[16] C. Ciccarelli, K. M. D. Hals, A. Irvine, V. Novak, Y. Tserkovnyak, H. Kurebayashi, A. Brataas, and A. Ferguson, Nature Nanotech. 10, 50 (2015).

[17] Shahrzad Karimi and C. A. Ullrich, Phys. Rev. B 90, 245304 (2014)

[18] S. Karimi, F. Baboux, F. Perez, C. A. Ullrich, G. Karczewski, and T. Wojtowicz, Phys. Rev. B 96, 045301 (2017)

[19] P. Hohenberg and W. Kohn, Phys. Rev 136, B864 (1964).

[20] W. Kohn and L. J. Sham, Phys. Rev. 140, A1133 (1965). 
[21] The usual Coulomb interaction, $1 /\left|\mathbf{r}-\mathbf{r}^{\prime}\right|$, applies in 3D and 2D, but in 1D it would lead to divergencies and requires regularization (see Ref [2].).

[22] L. Pollack and J. P. Perdew, J. Phys.: Condens. Matter 12, 1239 (2000).

[23] Y.-H. Kim, I.-H. Lee, S. Nagaraja, J.-P. Leburton, R. Q. Hood, and R. M. Martin, Phys. Rev. B 61, 5202 (2000).

[24] L. A. Constantin, J. P. Perdew, and J. M. Pitarke, Phys. Rev. Lett. 101, 016406 (2008).

[25] L. A. Constantin, Phys. Rev. B 78, 155106 (2008).

[26] J. F. Dobson, J. Wang, and T. Gould, Phys. Rev. B 66, 081108 (2002).

[27] J. F. Dobson, Phys. Chem. Chem. Phys. 11, 4528 (2009).

[28] E. Runge and E. K. U. Gross, Phys. Rev. Lett. 52, 997 (1984).

[29] C. A. Ullrich, Time-dependent density-functional theory: Concepts and applications (Oxford University Press, 2012).

[30] C. A. Ullrich and Zeng-hui Yang, Brazilian J. Phys. 44, 154 (2014).

[31] M. Petersilka, U. J. Gossmann, and E. K. U. Gross, Phys. Rev. Lett. 76, 1212 (1996).

[32] M. E. Casida, in Recent Advances in Density Functional Methods, edited by D. E. Chong, Vol. 1 of Recent Advances in Computational Chemistry (World Scientific, Singapore, 1995), p. 155. 
[33] Here we distinguish quasi-2D and strictly 2D systems. By quasi-2D we mean the quantum limit in which all electrons occupy the lowest subband in the well, but the electron density distribution still has a finite lateral width; strictly $2 \mathrm{D}$ implies the limit in which the width has become infinitesimal.

[34] E. K. U. Gross and W. Kohn, Phys. Rev. Lett. 55, 2850 (1985); Erratum: ibid. 57, $923(1986)$.

[35] A. Zangwill and P. Soven, Phys. Rev. A 21, 1561 (1980).

[36] J. F. Dobson, Phys. Rev. B 46, 10163 (1992).

[37] W. L. Schaich and J. F. Dobson, Phys. Rev. B 49, 14700 (1994).

[38] J. P. Perdew, K. Burke, and M. Ernzerhof, Phys. Rev. Lett. 77, 3865 (1996); Erratum: ibid. 78, 1396 (1997).

[39] M. Petersilka, U. J. Gossmann, and E. K. U. Gross, in Electronic density functional theory: recent progress and new directions, ed. by J. F. Dobson and G. Vignale (Plenum, New York, 1998), p. 177.

[40] N. H. March and M. P. Tosi, Adv. Phys. 44, 299 (1995).

[41] A. Liebsch, Electronic excitations at metal surfaces (Plenum Press, New York, 1997).

[42] A similar effect was described earlier by DasSarma and Marmorkos [Phys. Rev. B 47, 16343 (1993)], but it was not recognized as being an artifact of the 3D ALDA in the quasi-2D limit.

[43] A. Savin, C. J. Umrigar, and X. Gonze, Chem. Phys. Lett. 288, 391 (1998). 
[44] K. Burke, M. Petersilka, and E. K. U. Gross, in Recent Advances in Density Functional Methods, ed. V. Barone, A. Bencini, and P. Fantucci, Vol. III (World Scientific, Singapore, 2002), p. 67.

[45] However, there are molecular examples where the exact Kohn-Sham transitions do not lie between the singlet and triplet excitations, see E. J. Baerends, O. V. Gritsenko, and R. van Meer, Phys. Chem. Chem. Phys. 15, 16408 (2013).

[46] This would also affect the so-called hybrid functionals such as PBE0 [C. Adamo and V. Barone, J. Chem. Phys. 110, 6158 (1999)], which combine GGA functionals with nonlocal Hartree-Fock exchange. The nonlocal exchange part is wellbehaved, but the GGA part of the functional still causes problems going from $3 \mathrm{D}$ to $2 \mathrm{D}$. One may speculate that hybrid functionals might still work for well widths where pure GGAs would fail; however, nonlocal exchange is difficult to implement in quantum wells [M. S-C. Luo, S. L. Chuang, S. Schmitt-Rink, and A. Pinczuk, Phys. Rev. B 48, 11086 (1993)], so it may not be worth the effort.

[47] C. A. Ullrich and G. Vignale, Phys. Rev. B 58, 7141 (1998).

[48] C. A. Ullrich and G. Vignale, Phys. Rev. B 58, 15756 (1998).

[49] C. A. Ullrich and G. Vignale, Phys. Rev. Lett. 87, 037402 (2001).

[50] C. A. Ullrich and G. Vignale, Phys. Rev. B 65, 245102 (2002); Erratum: ibid. 70, 239903(E) (2004).

[51] G. Vignale and W. Kohn, Phys. Rev. Lett. 77, 2037 (1996).

[52] G. Vignale, C. A. Ullrich, and S. Conti, Phys. Rev. Lett. 79, 4878 (1997). 
[53] The Vignale-Kohn functional is a local functional of the current and, hence, a nonlocal functional of the density. In the 3D-2D crossover it would perform poorly for two reasons: its functional form is only valid for situations where the system and the perturbation are weakly inhomogeneous (which is clearly not the case in the 2D limit), and it contains locally evaluated xc viscosity coefficients of the 3D electron liquid.

[54] R. D’Agosta, M. Di Ventra and G. Vignale, Phys. Rev. B 76, 035320 (2007).

[55] I. D'Amico and C. A. Ullrich, Phys. Rev. B 74, 121303 (2006).

[56] I. D'Amico and C. A. Ullrich, Phys. Rev. B 88, 155324 (2013).

[57] L. D. Landau and E. M. Lifshitz, The classical theory of fields, 4th edition (Butterworth-Heinemann, Oxford, 1975).

[58] E. Lipparini, Modern many-particle physics, 2nd edition (World Scientific, Singapore, 2008).

[59] K. Yosida, Theory of magnetism (Springer, Berlin, 1996).

[60] D. Stein, K. v. Klitzing, and G. Weimann, Phys. Rev. Lett. 51, 130 (1983).

[61] J. P. Longo and C. Kallin, Phys. Rev. B 47, 4429 (1993).

[62] M. Califano, T. Chakraborty, P. Pietiläinen, and C.-M. Hu, Phys. Rev. B 73, 113315 (2006).

[63] Y.-T. Zhang, Z.-F. Song, and Y.-C. Li, Phys. Lett. A 373, 144 (2008).

[64] R. Roldán, J.-N. Fuchs, and M. O. Goerbig, Phys. Rev. B 82, 205418 (2010). 
[65] S. S. Krishtopenko, Semicond. Sci. Technol. 29, 085005 (2014).

[66] S. S. Krishtopenko, Semicond. 49, 174 (2015).

[67] S. Maiti, M. Imran, and D. L. Maslov, Phys. Rev. B 93, 045134 (2016).

[68] C. A. Ullrich and M. E. Flatté, Phys. Rev. B 66, 205305 (2002).

[69] C. A. Ullrich and M. E. Flatté, Phys. Rev. B 68, 235310 (2003).

[70] F. Baboux, F. Perez, C. A. Ullrich, I. D'Amico, J. Gomez, and M. Bernard, Phys. Rev. Lett. 109, 166401 (2012).

[71] F. Baboux, F. Perez, C. A. Ullrich, I. D'Amico, G. Karczewski, and T. Wojtowicz, Phys. Rev. B 87, 121303 (2013).

[72] F. Baboux, F. Perez, C. A. Ullrich, G. Karczewski, and T. Wojtowicz, Phys. Rev. B 92, 125307 (2015).

[73] F. Baboux, F. Perez, C. A. Ullrich, G. Karczewski, and T. Wojtowicz, Phys. Stat. Solidi RRL 10, 315 (2016).

[74] F. Perez, F. Baboux, C. A. Ullrich, I. D’Amico, G. Vignale, G. Karczewski, and T. Wojtowicz, Phys. Rev. Lett. 117, 137204 (2016).

[75] E. M. Lifshitz and L. P. Pitaevskii, Statistical Physics: Theory of the Condensed State (Butterworth-Heinemann, Oxford, 1980).

[76] F. Baboux, Effets Spin-Orbite Gants Sur Les Modes Collectifs de Spin de Puits Quantiques, PhD thesis, Institut des NanoSciences de Paris, Universit Pierre et Marie Curie (2013). 
[77] T. Holstein and H. Primakoff, Phys. Rev. 58, 1098 (1940).

[78] F. Perez, Phys. Rev. B 79, 045306 (2009)

[79] F. Perez, J. Cibert, M. Vladimirova, and D. Scalbert, Phys. Rev. B 83, 075311 (2011).

[80] C. Attaccalite, S. Moroni, P. Gori-Giorgi, and G. B. Bachelet, Phys. Rev. Lett. 88, 256601 (2002).

[81] F. Perez, C. Aku-leh, D. Richards, B. Jusserand, L. C. Smith, D. Wolverson and G. Karczewski, Phys. Rev. Lett. 99, 026403 (2007)

[82] A. K. Rajagopal, Phys. Rev. B 17, 2980 (1978).

[83] S. M. Badalyan, A. Matos-Abiague, G. Vignale, and J. Fabian, Phys. Rev. B 79, 205305 (2009).

[84] P. E. Iglesias and J. A. Maytorena, Phys. Rev. B 82, 205324 (2010).

[85] C. M. Wang and M. Q. Pang, Eur. Phys. J. B 74, 19 (2010).

[86] C. Li and F. Zhai, J. Appl. Phys. 109, 093306 (2011).

[87] A. Ashrafi and D. L. Maslov, Phys. Rev. Lett. 109, 227201 (2012).

[88] S. Maiti, V. Zyuzin, and D. L. Maslov, Phys. Rev. B 91, 035106 (2015).

[89] S. Maiti and D. L. Maslov, Phys. Rev. Lett. 114, 156803 (2015).

[90] J. P. Perdew and Y. Wang, Phys. Rev. B 45, 13244 (1992). 
[91] In our numerical calculations, the Mn magnetic moments are not included, which does not affect the electronic structure and dynamics. However, we need to compensate by using a larger magnetic field $(4.18 \mathrm{~T})$ compared to experiment $(2$ $\mathrm{T})$.

[92] Note that in Ref. [74] we obtained slightly different SOC parameters $(\alpha=1.83$ meVA and $\beta=3.79 \mathrm{meV} \AA$ ) by using the ALDA for $Z^{*}$ and fitting to $E_{1}$ only.

[93] P. S. Eldridge, J. Hübner, S. Oertel, R. T. Harley, M. Henini, and M. Oestreich, Phys. Rev. B 83, 041301(R) (2011).

[94] P. S. Alekseev, Semicond. 47, 1241 (2013).

[95] C. Rice, D. Wolverson, A. Moskalenko, S. J. Bending, G. Karczewski, and T. Wojtowicz, Phys. Rev. B 87, 121304(R) (2013).

[96] S. Sharma, J. K. Dewhurst, C. Ambrosch-Draxl, S. Kurth, N. Helbig, S. Pittalis, S. Shallcross, L. Nordström, and E. K. U. Gross, Phys. Rev. Lett. 98, 196405 (2007).

[97] G. Scalmani and M. J. Frisch, J. Chem. Theor. Comput. 8, 2193 (2012).

[98] F. G. Eich and E. K. U. Gross, Phys. Rev. Lett. 111, 156401 (2013).

[99] F. G. Eich, S. Pittalis, and G. Vignale, Phys. Rev. B 88, 245102 (2013).

[100] D. D. Stancil and A. Prabhakar, Spin waves: Theory and applications (Springer, Berlin, 2010).

[101] K. Zakeri, Phys. Rep. B 545, 47 (2014). 
[102] K. Zakeri, Y. Zhang, T.-H. Chuang, and J. Kirschner, Phys. Rev. Lett. 108, 197205 (2012).

[103] K. Di, V. L. Zhang, H. S. Lim, S. C. Ng, M. H. Kuok, J. Yu, J. Yoon, X. Qiu, and H. Yang, Phys. Rev. Lett. 114, 047201 (2015).

[104] M. W. Wu, J. H. Jiang, and M. Q. Weng, Physics Reports 493, 61 (2010).

[105] T. Liu and G. Vignale, Phys. Rev. Lett. 106, 247203 (2011).

[106] J. Schliemann, J. C. Egues, and D. Loss, Phys. Rev. Lett. 90, 146801 (2003).

[107] B. A. Bernevig, J. Orenstein, and S.-C. Zhang, Phys. Rev. Lett. 97, 236601 (2006).

[108] M.-H. Liu, K.-W. Chen, S.-H. Chen, and C.-R. Chang, Phys. Rev. B 74, 235322 (2006).

[109] J. D. Koralek, C. P. Weber, J. Orenstein, B. A. Bernevig, Shou-Cheng Zhang, S. Mack, and D. D. Awschalom, Nature 458, 610 (2009).

[110] M. P. Walser, C. Reichl, W. Wegscheider, and G. Salis, Nature Physics 8, 757 (2012).

[111] C. Schnhuber, M. P. Walser, G. Salis, C. Reichl, W. Wegscheider, T. Korn, and C. Schller, Phys. Rev. B 89, 85406 (2014).

[112] A. Sasaki, S. Nonaka, Y. Kunihashi, M. Kohda, T. Bauernfeind, T. Dollinger, K. Richter, and J. Nitta, Nature Nanotechnology 9, 703 (2014). 
[113] M. Kammermeier, P. Wenk, and J. Schliemann, Phys. Rev. Lett. 117, 236801 (2016).

[114] J. Schliemann, Rev. Mod. Phys. 89, 11001 (2017).

[115] M. Duckheim and D. Loss, Phys. Rev. B 75, 201305 (2007).

[116] E. P. Nakhmedov and O. Alekperov, Eur. Phys. J. B 85, 298 (2012).

[117] M. A. Wilde and D. Grundler, New J. Phys. 15, 115013 (2013).

[118] R. G. Nazmitdinov, K. N. Pichugin, and M. Valn-Rodrguez, Phys. Rev. B 79, 193303 (2009).

[119] C. Micu and E. Papp, Superlattices and Microstructures 51, 651 (2012).

[120] Y. Au, E. Ahmad, O. Dmytriiev, M. Dvornik, T. Davison, and V. V. Kruglyak, Appl. Phys. Lett. 100, 182404 (2012).

[121] H. Yu, G. Duerr, R. Huber, M. Bahr, T. Schwarze, F. Brandl, and D. Grundler, Nature Communications 4, 2702 (2013).

[122] F. B. Mushenok, R. Dost, C. S. Davies, D. A. Allwood, B. J. Inkson, G. Hrkac, and V. V. Kruglyak, Appl. Phys. Lett. 111, 42404 (2017).

[123] K. S. Singwi, M. P. Tosi, R. H. Land, and A. Sjolander, Phys. Rev. 176, 589 (1968). 


\section{VITA}

Shahrzad Karimi was born on March 28, 1990 in Tehran, Iran. She received her Bachelor of Science degree in Physics from the University of Tehran, Iran in August 2012. She attended Physics and Astronomy Department at the University of Missouri in fall 2012, and started to work under supervision of Prof. Carsten Ullrich. She received her Master's and PhD degrees in Theoretical and Computational Physics in 2015 and 2017, respectively. She will start working as a Computational Physicist in industry after graduation.

\section{Publication List}

1. Three-to two-dimensional crossover in time-dependent density-functional theory, Shahrzad Karimi and C. A. Ullrich, Phys. Rev. B 90, 245304 (2014)

2. Spin precession and spin waves in a chiral electron gas: beyond Larmor's theorem, S. Karimi, F. Baboux, F. Perez, C. A. Ullrich, G. Karczewski, and T. Wojtowicz, Phys. Rev. B 96, 045301 (2017)

3. Spin-helix Larmor mode, S. Karimi, C. A. Ullrich, I. D'Amico, and F. Perez, Submitted to Scientific Reports (2017) 